\title{
Equivariant principal bundles and their classifying spaces
}

\author{
WOLFGANG LÜCK \\ BERNARDO URIBE
}

\begin{abstract}
We consider $\Gamma$-equivariant principal $G$-bundles over proper $\Gamma$-CW-complexes with a prescribed family of local representations. We construct and analyze their classifying spaces for locally compact, second countable topological groups $\Gamma$ and $G$ with finite covering dimensions, where $G$ is almost connected.
\end{abstract}

$55 \mathrm{R} 91 ; 55 \mathrm{P} 91$

\section{Introduction}

Let $\Gamma$ and $G$ be topological groups. We will introduce the notion of a $\Gamma$-equivariant principle $G$-bundle over a $\Gamma-\mathrm{CW}$-complex, ie a principal $G$-bundle $p: E \rightarrow B$ together with left $\Gamma$-actions on $E$ and $B$ commuting with the right $G$-action on $E$ such that $p$ is $\Gamma$-equivariant. For every $e \in E$ we obtain a local representation $\rho_{e}: \Gamma_{p(e)} \rightarrow G$ uniquely determined by $\gamma^{-1} \cdot e=e \cdot \rho_{e}(\gamma)$ for $\gamma \in \Gamma_{p(e)}$, where $\Gamma_{p(e)}$ is the isotropy group of $p(e) \in B$. One can consider such bundles where the family of local representations $\mathcal{R}$ is prescribed, eg one may demand that $\Gamma_{p(e)}$ is always compact and allow only certain homomorphisms $\rho_{e}$.

Our main technical result is Theorem 8.1, where we prove that a $\Gamma$-equivariant principal $G$-bundle $p: E \rightarrow B$ is the same as a $\Gamma \times G-\mathrm{CW}$-complex $E$, provided that the family of locally representations satisfies Condition $(H)$ introduced in Definition 6.1. This implies the main result of this paper (Theorem 11.4) which gives a universal $\Gamma$-equivariant principal $G$-bundle with respect to a given family of local representations $\mathcal{R}$, provided that $\mathcal{R}$ satisfies Condition $(\mathrm{H})$.

Condition $(\mathrm{H})$ is needed to ensure homotopy invariance for $\Gamma$-equivariant principal $G-$ bundles. It is automatically satisfied if $\Gamma$ and $G$ are locally compact second countable groups with finite covering dimensions (eg Lie groups), $G$ is almost connected (ie $G$ modulo its connected component of the identity is compact) and all base spaces are $\Gamma-\mathrm{CW}-$ complexes with compact isotropy groups; see Theorem 6.3. 
Equivariant principal bundles have been studied before by several authors (see Hambleton and Hausmann [9], Lashof [18], Lashof and May [19], Lashof, May and Segal [20], May [24], Maruyama and Shimakawa [28] and tom Dieck [7] and references therein) and our construction generalizes all the previous constructions in the following sense. We isolated the conditions that the groups $\Gamma$ and $G$ and the family of local representations need to satisfy in order to ensure the existence of a universal equivariant bundle associated to the prescribed family of local representations as base; this is the Condition $(\mathrm{H})$ mentioned above. Moreover, in the literature some conditions about the restriction of the bundles to $\Gamma$-invariant neighborhoods of points in the base space are demanded; we show that they automatically follow from our setting in Theorem 9.1 if the Condition (H) is satisfied.

In Section 15 we have included a study of the case $G=\mathcal{P U}(\mathcal{H})$, the projective unitary group, endowed with the norm topology. The main result in Section 15 is Theorem 15.12, which produces a universal $\Gamma$-equivariant stable projective unitary bundle for almost free $\Gamma-\mathrm{CW}$-complexes. This result generalizes Barcenas, Espinoza, Joachim and the second author's [5, Theorem 3.21], where $\Gamma$ is assumed to be discrete. This universal bundle is relevant for equivariant twisted topological $K$-theory since with this bundle it can be defined as a parameterized equivariant cohomology theory.

Our results carry directly over to the case where one allows an intertwining between the $\Gamma$ - and the $G$-action, ie there exists a group homomorphism $\tau: \Gamma \rightarrow \operatorname{aut}(G)$ and the condition that the $\Gamma$ - and $G$-action on the total space commute is replaced by the weaker condition $\gamma \cdot(e \cdot g)=(\gamma \cdot e) \cdot \tau(\gamma)(g)$. A typical example for such a nontrivial intertwining is the case $\Gamma=\mathbb{Z} / 2, G=U(n)$ and $\tau: \mathbb{Z} / 2 \rightarrow \operatorname{aut}(U(n))$ given by complex conjugation, which leads to real vector bundles in the sense of Atiyah [2]. For the simplicity of the exposition we only treat the case where $\tau$ is trivial.

Throughout this paper we will work in the category of compactly generated spaces (see Steenrod [33] and Appendix A) and subgroups are always understood to be closed subgroups. Most of the equivariant CW-complexes under consideration are proper, or, equivalently, have compact isotropy groups.

Acknowledgements This paper has been financially supported by the Leibniz Award, granted by the Deutsche Forschungsgemeinschaft, to the first author, and by the Alexander Von Humboldt Foundation through a scholarship for Experienced Researchers granted to the second author. Both authors would like to acknowledge and thank the financial support of the Deutsche Forschungsgemeinschaft and of the Alexander Von Humboldt Foundation. Moreover, the authors thank the referee for his careful reading of the first version and his useful comments. 


\section{Principal bundles}

We recall some basic facts about principal $G$-bundles over CW-complexes for a topological group $G$.

Definition 1.1 (Quasiregular open set and regular space) An open subset $U \subseteq B$ is called quasiregular if for any $x \in U$ there exists an open neighborhood $V_{x}$ whose closure in $B$ is contained in $U$. An space is called regular if it separates points from closed subsets.

The main point of this notion is that a quasiregular open subset equipped with its subspace topology is again compactly generated (see Lemma A.1(i)) and preimages of quasiregular open subsets are again quasiregular open subsets; see Lemma A.1(ii).

Definition 1.2 (Principal $G$-bundle) A principal $G$-bundle $p: E \rightarrow B$ consists of a space $E$ with right $G$-action, a space $B$ with trivial $G$-action and a $G$-map $p$ such that $p$ is locally trivial, ie for any $b \in B$ there exists a quasiregular open neighborhood $U$ of $b$ in $B$ and a $G$-homeomorphism $\phi: G \times U \rightarrow p^{-1}(U)$ satisfying $p \circ \phi=$ pr for the projection pr: $U \times G \rightarrow U$.

In the previous definition we have added the condition that the local trivialization can be done on a quasiregular open set. On the standard definition of principal bundles the quasiregularity is not required. Nevertheless, since in this article we will work in the category of equivariant $\mathrm{CW}$-complexes, and any invariant open subset of an equivariant CW-complex is automatically quasiregular (see Lemma A.1(vi)) this extra condition in the definition is innocuous.

Lemma 1.3 Let $B$ be a $C W-$ complex and let $p: E \rightarrow B \times[0,1]$ be a principal $G$-bundle. Let $i_{0}: B=B \times\{0\} \rightarrow B \times[0,1]$ be the inclusion.

Then $i_{0}^{*} p \times \operatorname{id}_{[0,1]}: i_{0}^{*} E \times[0,1] \rightarrow B \times[0,1]$ is a principal $G$-bundle and there exists an isomorphism of principal $G$-bundles

$$
f: i_{0}^{*} E \times[0,1] \rightarrow E
$$

over $B \times[0,1]$ whose restriction to $B \times\{0\}$ is the identity.

Proof A CW-complex $B$ is paracompact by Miyazaki [25]. Now the proof is analogous to the one of Husemoller [11, Theorem 9.8 in Chapter 3 on page 51] taking into account that any open subset of $B$ is quasiregular by Lemma A.1(iv) and (v) and that in [11, Theorem 9.8 in Chapter 3 on page 51] the symbol $\times$ stands for the classical product space, where in our setting $\times$ stands for the product within the category of compactly generated spaces. 
Notation 1.4 Given a $\Gamma \times G$-space $X$, let $X_{r}$ be the space $X$ but now equipped with the left $\Gamma$ action given by $\gamma \cdot x=(\gamma, 1) \cdot x$ and the right $G$-action given by $x \cdot g:=\left(1, g^{-1}\right) \cdot x$.

Given a space $Y$ with (commuting) left $\Gamma$ - and right $G$-action, let $Y_{l}$ be the same space but now equipped with the left $\Gamma \times G$-action given by $(\gamma, g) \cdot y:=\gamma \cdot y \cdot g^{-1}$.

Example 1.5 (Free $G$-CW-complexes) Let $X$ be a free $G-\mathrm{CW}-$ complex. Then $p: X_{r} \rightarrow X_{r} / G$ is a principal $G$-bundle. Conversely, if $p: E \rightarrow B$ is a principal $G-$ bundle over a CW-complex $B$, then $E_{l}$ carries the structure of a free $G-\mathrm{CW}$-complex coming from the filtration given by the preimages of the skeletons of $B$.

These claims are proved by the first author in [22, 1.24 and 1.25 on page 18], and they will also follow from Theorem 8.1 applied to the special case $\Gamma=\{1\}$.

Example 1.6 (Free proper smooth $G$-actions on smooth manifolds) Consider a Lie group $G$ with a free proper smooth left $G$-action on a smooth manifold $M$. Then $M$ is a proper $G-\mathrm{CW}-$ complex by Illman [12] and the projection $M_{r} \rightarrow M_{r} / G$ is a principal $G$-bundle.

\section{Equivariant principal bundles}

We first fix the notions for the objects we want to study.

Definition 2.1 ( $\Gamma$-equivariant principle $G$-bundle) A $\Gamma$-equivariant principal $G$ bundle $p: E \rightarrow B$ consists of a principal $G$-bundle together with left $\Gamma$-actions on $E$ and $B$ (commuting with the right $G$-actions) such that $p: E \rightarrow B$ is $\Gamma$-equivariant.

Note that since the $\Gamma$ and the $G$ actions commute, then $\Gamma$ acts on $p: E \rightarrow B$ through $G$-bundle maps, cf [18, Section 1]; see also [7, Chapter I, Section 8] for this notion and its main properties including universal objects for a compact Lie group $\Gamma$ and a topological group $G$, where also a twisting of the left $\Gamma$ - and right $G$-actions by a homomorphism $\Gamma \rightarrow \operatorname{aut}(G)$ is allowed. Sometimes in the literature some conditions about the restriction of the bundles to $\Gamma$-invariant neighborhoods of points in the base space are demanded or can only be proved in the case that $G$ is a compact Lie group; see for instance [7, Proposition 8.10 on page 58]. We will show that they automatically follow from our setting in Theorem 9.1.

We mention some basic properties of $\Gamma$-equivariant principal $G$-bundles. 
If $f: X \rightarrow B$ is a $\Gamma$-map of $\Gamma-\mathrm{CW}$-complexes and $p: E \rightarrow B$ is a $\Gamma$-invariant principal $G$-bundle, then the map $f^{*} p: f^{*} E \rightarrow X$ obtained from the pullback of $p$ with $f$,

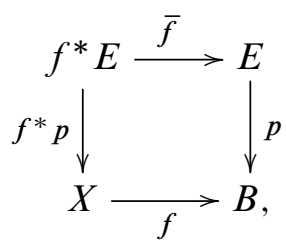

is a $\Gamma$-equivariant principal $G$-bundle again. An isomorphism of two $\Gamma$-invariant principal $G$-bundles $p_{0}: E_{0} \rightarrow X$ and $p_{1}: E_{1} \rightarrow B$ over the $\Gamma-C W-$ complex $B$ is a homeomorphism $f: E_{0} \rightarrow E_{1}$ which is compatible with both the left $\Gamma$-action and the right $G$-action and satisfies $p_{1} \circ f=p_{0}$.

Lemma 2.2 Let $p_{0}: E_{0} \rightarrow B$ and $p_{1}: E_{1} \rightarrow B$ be $\Gamma$-equivariant principle $G$-bundles over the $\Gamma-C W$-complex $B$. Let $f: E_{0} \rightarrow E_{1}$ be a map which is compatible with both the left $\Gamma$-actions and the right $G$-actions and satisfies $p_{1} \circ f=p_{0}$.

Then $f$ is an isomorphism of $\Gamma$-equivariant principal $G$-bundles.

Proof The map $f$ is a homeomorphism because of the local triviality of the principal $G$-bundles $p_{0}$ and $p_{1}$.

\section{Families of local representations}

In this section we introduce the local representations coming from the left action of the $\Gamma$-isotropy group of a point $b$ in the base space and the free right $G$-action on the fiber over $b$ (after choosing a lift of $b$ to the total space).

Definition 3.1 (Local representations) Let $p: E \rightarrow B$ be a $\Gamma$-equivariant principal $G$-bundle. Consider $e \in E$. Then we obtain a (continuous) group homomorphism

$$
\rho_{e}: \Gamma_{p(e)} \rightarrow G
$$

uniquely determined by $\gamma \cdot e=e \cdot \rho_{e}(\gamma)$ for $\gamma \in \Gamma_{p(e)}$, where $\Gamma_{p(e)}$ is the isotropy group of $p(e) \in B$.

The homomorphism $\rho_{e}$ is indeed a homomorphism by the calculation

$$
\begin{aligned}
e \cdot \rho_{e}\left(\gamma_{1} \cdot \gamma_{2}\right) & =\left(\gamma_{1} \cdot \gamma_{2}\right) \cdot e=\gamma_{1} \cdot\left(\gamma_{2} \cdot e\right) \\
& =\gamma_{1} \cdot\left(e \cdot \rho_{e}\left(\gamma_{2}\right)\right)=\left(\gamma_{1} \cdot e\right) \cdot \rho_{e}\left(\gamma_{2}\right) \\
& =\left(e \cdot \rho_{e}\left(\gamma_{1}\right)\right) \cdot \rho_{e}\left(\gamma_{2}\right)=e \cdot\left(\rho_{e}\left(\gamma_{1}\right) \cdot \rho_{e}\left(\gamma_{2}\right)\right) .
\end{aligned}
$$


It is continuous since the map $G \rightarrow p^{-1}(e), g \mapsto e \cdot g$ is a homeomorphism because of the local triviality of the principal $G$-bundle $p$ and the map $\Gamma_{p(e)} \rightarrow p^{-1}(e), \gamma \mapsto \gamma \cdot e$ is continuous.

Remark 3.2 (Basic properties of the local representations) If we replace $e$ by $e g$ for some $g \in G$, then $\rho_{e g}=c_{g^{-1}} \circ \rho_{e}$ for $c_{g}: G \rightarrow G$ the conjugation homomorphism sending $g^{\prime}$ to $g g^{\prime} g^{-1}$.

If we replace $e$ by $\gamma e$ for some $\gamma \in \Gamma$, then $\Gamma_{p(e)}=\gamma^{-1} \Gamma_{p(\gamma e)} \gamma$ and $\rho_{\gamma e}=\rho_{e} \circ c_{\gamma^{-1}}$. If

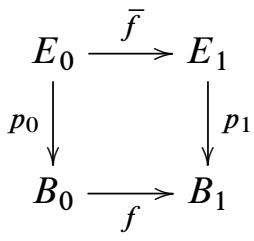

is a morphism of $\Gamma$-equivariant principal $G$-bundles, then

$$
\rho_{e}^{p_{0}}=\rho_{\bar{f}(e)}^{p_{1}} \circ i_{e}
$$

holds for all $e \in E_{0}$, where $i_{e}: \Gamma_{p_{0}(e)} \rightarrow \Gamma_{p_{1} \circ \bar{f}(e)}$ is the inclusion.

Definition 3.3 (Family of local representations) A family $\mathcal{R}$ of local representations for $(\Gamma, G)$ is a set of pairs $(H, \alpha)$, where $H$ is a subgroup of $\Gamma$ and $\alpha: H \rightarrow G$ is a continuous group homomorphism such that the following conditions are satisfied.

- Finite intersections Suppose that $\left(H_{0}, \alpha_{0}\right)$ and $\left(H_{1}, \alpha_{1}\right)$ belong to $\mathcal{R}$. Define $H:=\left\{h \in H_{0} \cap H_{1} \mid \alpha_{0}(h)=\alpha_{1}(h)\right\}$ and $\alpha: H \rightarrow G$ by $\alpha=\left.\alpha_{0}\right|_{H}=\left.\alpha_{1}\right|_{H}$. Then $(H, \alpha) \in \mathcal{R}$.

- Conjugation in $G$ If $(H, \alpha)$ belongs to $\mathcal{R}$ and $g \in G$, then $\left(H, c_{g^{-1}} \circ \alpha\right)$ belongs to $\mathcal{R}$.

- Conjugation in $\Gamma$ If $(H, \alpha)$ belongs to $\mathcal{R}$ and $\gamma \in \Gamma$, then $\left(\gamma H \gamma^{-1}, \alpha \circ c_{\gamma^{-1}}\right)$ belongs to $\mathcal{R}$.

Definition 3.4 ((Pre)family of local representations associated to a $\Gamma$-equivariant principal $G$-bundle) Let $p: E \rightarrow B$ be a $\Gamma$-equivariant principal $G$-bundle. Define the prefamily of local representations of $p$ to be

$$
\mathcal{R}^{\prime}(p):=\left\{\left(\Gamma_{p(e)}, \rho_{e}\right) \mid e \in E\right\} .
$$

Let $\mathcal{R}(p)$ be the smallest system of local representations containing $\mathcal{R}^{\prime}(p)$. We call $\mathcal{R}(p)$ the family of local representations associated to $p$. 
One easily checks using Remark 3.2 that $\mathcal{R}^{\prime}(p)$ is closed under conjugation, but not necessary under finite intersections so that $\mathcal{R}^{\prime}(p)$ itself is not a family of local representations.

We want to deal with families of local representations to ensure that the following lemma is true.

A family of subgroups of $G$ is a set of subgroups of $G$ closed under conjugation and taking finite intersections.

Lemma 3.5 Let $\mathcal{R}$ be a family of local representations for $\Gamma$ and $G$. For $(H, \alpha)$ in $\mathcal{R}$ let $K(H, \alpha)$ be the subgroup of $\Gamma \times G$ given by

$$
K(H, \alpha):=\{(\gamma, \alpha(\gamma)) \mid \gamma \in H\}
$$

Put

$$
\mathcal{F}(\mathcal{R})=\{K(H, \alpha) \mid(H, \alpha) \in \mathcal{R}\}
$$

Then $\mathcal{F}(\mathcal{R})$ is a family of subgroups of $\Gamma \times G$.

Proof We have to check that $\mathcal{F}(\mathcal{R})$ is closed under conjugation and finite intersections. Consider $K \in \mathcal{F}(\mathcal{R})$ and $(\gamma, g) \in \Gamma \times G$. Choose $(H, \alpha) \in \mathcal{R}$ with $K=K(H, \alpha)$. Then

$$
\begin{aligned}
(\gamma, g)^{-1} \cdot K \cdot(\gamma, g) & =(\gamma, g)^{-1} \cdot K(H, \alpha) \cdot(\gamma, g) \\
& =\left\{(\gamma, g)^{-1} \cdot(h, \alpha(h)) \cdot(\gamma, g) \mid h \in H\right\} \\
& =\left\{\left(\gamma^{-1} h \gamma, g^{-1} \alpha(h) g\right) \mid h \in H\right\} \\
& =\left\{\left(c_{\gamma}(h), c_{g^{-1}} \circ \alpha \circ c_{\gamma}\left(c_{\gamma^{-1}}(h)\right) \mid h \in H\right\}\right. \\
& =\left\{h^{\prime}, c_{g^{-1}} \circ \alpha \circ c_{\gamma}\left(h^{\prime}\right) \mid h^{\prime} \in \gamma^{-1} H \gamma\right\} \\
& =K\left(\gamma^{-1} H \gamma, c_{g^{-1}} \circ \alpha \circ c_{\gamma}\right) .
\end{aligned}
$$

Since $\left(\gamma^{-1} H \gamma, c_{g^{-1}} \circ \alpha \circ c_{\gamma}\right)$ belongs to $\mathcal{R}$, we conclude that $(\gamma, g)^{-1} \cdot K \cdot(\gamma, g)$ belongs to $\mathcal{F}(\mathcal{R})$.

Consider $K_{0}, K_{1} \in \mathcal{F}(\mathcal{R})$. Choose $\left(H_{i}, \alpha_{i}\right)$ in $\mathcal{R}$ with $K_{i}=K\left(H_{i}, \alpha_{i}\right)$ for $i=0,1$. Define $H:=\left\{h \in H_{0} \cap H_{1} \mid \alpha_{0}(h)=\alpha_{1}(h)\right\}$ and $\alpha: H \rightarrow G$ by $\alpha=\left.\alpha_{0}\right|_{H}=\left.\alpha_{1}\right|_{H}$. Then $K_{0} \cap K_{1}=K(H, \alpha)$ and $(H, \alpha) \in \mathcal{R}$. This implies $K_{0} \cap K_{1} \in \mathcal{F}(\mathcal{R})$.

Remark 3.6 (Families) We later will consider the classifying space $E_{\mathcal{F}}(\Gamma)$ of a family of subgroups of $\Gamma$. It can be defined without the condition that $\mathcal{F}$ is closed under finite intersections (being closed under conjugation is enough) but this extra 
condition is usually required to ensure that for two $\Gamma$-spaces $X$ and $Y$ whose isotropy groups belong to $\mathcal{F}$ also the isotropy groups of $X \times Y$ with the diagonal $\Gamma$-action belong to $\mathcal{F}$.

It is actually more convenient to require instead of the condition that $\mathcal{F}$ is closed under finite intersections that it is closed under subgroups. However, this rules out one important case, namely, the case of the family of open compact subgroups, which naturally occurs in the context of locally compact second countable totally disconnected groups $\Gamma$.

If $\mathcal{R}$ is closed under subgroups, ie, for $(H, \alpha) \in \mathcal{R}$ and any subgroup $K \subseteq H$ we have $\left(K,\left.\alpha\right|_{K}\right) \in \mathcal{R}$, then $\mathcal{F}(\mathcal{R})$ is closed under taking subgroups.

Remark 3.7 (Local representations and pullbacks) Let $p: E \rightarrow B$ be a $\Gamma$-equivariant principal $G$-bundle and let $f: A \rightarrow B$ be a $\Gamma$-map. Let $\mathcal{R}$ be a family of local representations. Suppose that we have $\mathcal{R}(p) \subseteq \mathcal{R}$. Then we get $\mathcal{R}\left(f^{*} p\right) \subseteq \mathcal{R}$ for the pullback $f^{*} p$, provided for any $(H, \alpha) \in \mathcal{R}$ and any subgroup $K \subseteq H$ which occurs as isotropy group in $A$, we have $\left(K,\left.\alpha\right|_{K}\right) \in \mathcal{R}$. This follows from Remark 3.2.

If we make the assumption that $\mathcal{R}$ is closed under subgroups, then $f^{*} p$ automatically satisfies $\mathcal{R}\left(f^{*} p\right) \subseteq \mathcal{R}$ if $\mathcal{R}(p) \subseteq \mathcal{R}$ holds.

\section{Condition (S)}

Definition 4.1 (Condition (S)) Given a topological group $\Gamma$ and a (closed) subgroup $H \subseteq \Gamma$, we say that the pair $(\Gamma, H)$ satisfies Condition $(S)$ if the projection pr: $\Gamma \rightarrow \Gamma / H$ has a local cross section, ie there is a quasiregular open neighborhood $U$ of $1 H \in \Gamma / H$ together with a map $\sigma: U \rightarrow \Gamma$ such that $\operatorname{pr} \circ \sigma=\mathrm{id}_{U}$.

A topological group $\Gamma$ satisfies Condition $(S)$ if for any subgroup $H \subseteq \Gamma$ the pair $(\Gamma, H)$ satisfies Condition $(\mathrm{S})$.

The role of the Condition (S) is to ensure the following lemma.

Lemma 4.2 Let $f: E \rightarrow \Gamma / H$ be a $\Gamma$-map for some subgroup $H \subseteq \Gamma$. Suppose that the pair $(\Gamma, H)$ satisfies Condition $(S)$. Then the $\Gamma-$ map

$$
u: \Gamma \times_{H} f^{-1}(1 H) \rightarrow E, \quad(\gamma, e) \mapsto \gamma \cdot e
$$

is a homeomorphism. 
Proof The map $u$ is clearly a bijective map of sets. Now, the Condition (S) ensures that there is an open neighborhood $U \subseteq \Gamma / H$ of $1 H$ and a map $s: U \rightarrow \Gamma$ whose composite with the projection pr: $\Gamma \rightarrow \Gamma / H$ is the identity on $U$. Moreover, the open subsets $U \subseteq \Gamma / H, \operatorname{pr}^{-1}(U) \subseteq \Gamma$ and $f^{-1}\left(\operatorname{pr}^{-1}(U)\right) \subseteq E$ equipped with the subspace topologies are compactly generated; see Lemma A.1(i) and (ii). Define a map

$$
v: f^{-1}\left(\operatorname{pr}^{-1}(U)\right) \rightarrow \operatorname{pr}^{-1}(U) \times_{H} f^{-1}(1 H), \quad e \mapsto\left(s \circ \pi(e), s \circ \pi(e)^{-1} \cdot e\right),
$$

where $\pi: f^{-1}\left(\mathrm{pr}^{-1}(U)\right) \rightarrow U$ is the map induced by prof . Let

$$
\left.u\right|_{\operatorname{pr}^{-1}(U) \times_{H} f^{-1}(1 H)}: \operatorname{pr}^{-1}(U) \times_{H} f^{-1}(1 H) \rightarrow f^{-1}\left(\operatorname{pr}^{-1}(U)\right)
$$

be obtained by restricting $u$. Then $\left.u\right|_{\mathrm{pr}^{-1}(U) \times_{H} f^{-1}(1 H)} \circ v=\mathrm{id}_{f^{-1}\left(\mathrm{pr}^{-1}(U)\right)}$ and

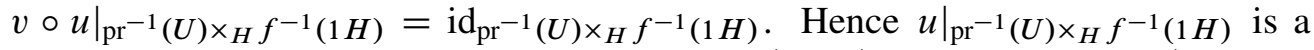
homeomorphism. Since $u$ is $\Gamma$-equivariant, $f^{-1}\left(\operatorname{pr}^{-1}(U)\right) \subseteq E$ and $\operatorname{pr}^{-1}(U) \times_{H}$ $f^{-1}(1 H) \subseteq \Gamma \times{ }_{H} f^{-1}(1 H)$ are open subsets, $\{\gamma \cdot U \mid \gamma \in \Gamma\}$ is an open covering of $\Gamma / H$ and $u$ is bijective, then the map $u$ is a homeomorphism.

Remark 4.3 (Condition (S) and principal bundle structure) If pr: $\Gamma \rightarrow \Gamma / H$ is a principal $H$-bundle, then the local triviality implies that the pair $(\Gamma, H)$ satisfies Condition (S). The converse is also true, namely, apply Lemma 5.1 (i) in the special case, where the role of $\Gamma, H$ and $G$ is played by $\Gamma, H$ and $H$, and $Z=\bullet$, and use the canonical $\Gamma$-homeomorphism $\Gamma \times_{H} H \stackrel{\cong}{\rightrightarrows} \Gamma,(\gamma, h) \mapsto \gamma \cdot h$.

Condition (S) is satisfied in many cases.

Lemma 4.4 (i) Suppose that $\Gamma$ is completely regular, ie for any $x \in \Gamma$ and any neighborhood $U$ of $x$ in $\Gamma$, there exists a continuous function $f: \Gamma \rightarrow[0,1]$ with $f(x)=0$ and $f(X \backslash U)=1$. Then for any subgroup $H \subseteq \Gamma$ which is a compact Lie group, the pair $(\Gamma, H)$ satisfies Condition $(S)$.

(ii) A topological group $\Gamma$ satisfies Condition $(S)$ if $\Gamma$ is discrete, if $\Gamma$ is a Lie group, or more generally, if $\Gamma$ is locally compact and second countable and has finite covering dimension.

Proof (i) This follows from Palais [30]; see also [22, Theorem 1.38 on page 27]. Notice that the conditions that $\Gamma$ is completely regular (and hence regular) and $H$ is compact imply that $\Gamma / H$ is regular. Hence every open subset of $\Gamma / H$ is quasiregular; see Lemma A.1(iv).

(ii) This follows from Mostert [26]. The metric needed in [26] follows under our assumptions from Theorem B.3. Notice that the condition that $\Gamma$ is locally compact implies that $\Gamma / H$ is locally compact and hence that any open subset of $\Gamma / H$ is quasiregular; see Lemma A.1(i) (iv) and (v). 
Example 4.5 (Kac-Moody groups) Kac-Moody groups are not Lie groups but all their compact subgroups are Lie groups; see Kitchloo [15, Theorem 2.4]. Since they are completely regular, Lemma 4.4(i) applies to them and therefore they satisfy Condition (S).

\section{Equivariant principal bundles over equivariant cells}

In this section we want to analyze $\Gamma$-equivariant principal $G$-bundles over spaces of the type $\Gamma / H \times Z$ for some subgroup $H \subset \Gamma$ and space $Z$ with trivial $H$-action. Later we will be mainly interested in the case $\Gamma / H \times D^{n}$.

Let $H$ and $G$ be two topological groups, and equip $\operatorname{hom}(H, G)$ with the subspace topology with respect to the inclusion $\operatorname{hom}(H, G) \subseteq \operatorname{map}(H, G)$; see Section A.5 for details.

Consider a space $Z$ and a map $\sigma: Z \rightarrow \operatorname{hom}(H, G)$. We have the obvious right $H$-action on $\Gamma$ and the left $H$-action on $Z \times G$ given by $h \cdot(z, g):=(z, \sigma(z)(h) \cdot g)$. Let

$$
p_{\sigma}: \Gamma \times{ }_{H}(Z \times G) \rightarrow \Gamma / H \times Z
$$

be the map induced by the projection $Z \times G \rightarrow Z$. It is compatible with the left $\Gamma$ action on $\Gamma \times{ }_{H}(Z \times G)$ given by $\gamma_{0} \cdot(\gamma,(z, g))=\left(\gamma_{0} \gamma,(z, g)\right)$ and the left $\Gamma$-action on $\Gamma / H \times Z$ given by $\gamma_{0} \cdot(\gamma \cdot H, z)=\left(\gamma_{0} \gamma \cdot H, z\right)$. It is also compatible with the right $G$-action on $\Gamma \times{ }_{H}(Z \times G)$ given by $(\gamma,(z, g)) \cdot g_{0}=\left(\gamma,\left(z, g g_{0}\right)\right)$ and the trivial right $G$-action on $\Gamma \times{ }_{H} Z$. The left $\Gamma$-action and the right $G$-action on $\Gamma \times{ }_{H}(Z \times G)$ commute.

Lemma 5.1 Suppose that the pair $(\Gamma, H)$ satisfies Condition ( $S$ ) (see Definition 4.1) and let $Z$ be a space. Then:

(i) The map $p_{\sigma}: \Gamma \times{ }_{H}(Z \times G) \rightarrow \Gamma / H \times Z$ is a $\Gamma$-equivariant principal $G$-bundle.

(ii) A $\Gamma$-equivariant principal $G$-bundle $E \rightarrow \Gamma / H \times Z$ is isomorphic as an $\Gamma$ equivariant principal $G$-bundle to the map $p_{\sigma}$ for an appropriate map $\sigma: Z \rightarrow$ $\operatorname{hom}(H, G)$, provided that the restriction of $p$ to $\{1 H\} \times Z$ is (after forgetting the $H$-action) a trivial principal $G-$ bundle.

(iii) Given two maps $\sigma_{0}: Z \rightarrow \operatorname{hom}(H, G)$ and $\sigma_{1}: Z \rightarrow \operatorname{hom}(H, G)$, we have that the $\Gamma$-equivariant principal $G$-bundles $p_{\sigma_{0}}$ and $p_{\sigma_{1}}$ are isomorphic if and only if there is a map $\omega: Z \rightarrow G$ such that

$$
\sigma_{1}(z)(g)=\omega(z) \cdot \sigma_{0}(z)(h) \cdot \omega(z)^{-1}
$$

for all $h \in H$ and $z \in Z$. 
(iv) Given a map $\sigma: Z \rightarrow \operatorname{hom}(H, G)$, the homomorphism $\rho_{(\gamma,(z, g))}: \Gamma_{(\gamma H, z)} \rightarrow G$ associated to $p_{\sigma}$ in (3-1) for $(\gamma,(z, g)) \in \Gamma \times \times_{H}(Z \times G)$ is given by

$$
\begin{gathered}
\Gamma_{(\gamma H, z)}=\gamma H \gamma^{-1}, \\
\rho_{(\gamma,(z, g))}\left(\gamma \cdot h \cdot \gamma^{-1}\right)=g^{-1} \sigma(z)(h) \cdot g .
\end{gathered}
$$

Proof (i) It remains to show that $p_{\sigma}: \Gamma \times{ }_{H}(Z \times G) \rightarrow \Gamma / H \times Z$ is a principal $G$-bundle after forgetting the $\Gamma$-action. The Condition (S) ensures that there is a quasiregular open neighborhood $U \subseteq \Gamma / H$ of $1 H$ and a map $s: U \rightarrow \Gamma$ whose composite with the projection pr: $\Gamma \rightarrow \Gamma / H$ is the identity. Notice that the open subsets $U \subseteq \Gamma / H$ and $\operatorname{pr}^{-1}(U) \subseteq \Gamma$ equipped with the subspace topology are compactly generated by Lemma A.1(i) and (ii).

Define $\bar{s}: \operatorname{pr}^{-1}(U) \rightarrow H$ by $\bar{s}(\gamma)=s \circ \operatorname{pr}(\gamma)^{-1} \cdot \gamma$. It has the property $\bar{s}(\gamma \cdot h)=\bar{s}(\gamma) \cdot h$ for all $\gamma \in p^{-1}(U)$ and $h \in H$. Define maps

$$
\begin{aligned}
\alpha:(U \times Z) \times G & \rightarrow \operatorname{pr}^{-1}(U) \times_{H}(Z \times G), \\
\beta: \operatorname{pr}^{-1}(U) \times_{H}(Z \times G) & \rightarrow(U \times Z) \times G,
\end{aligned}
$$

by

$$
\begin{aligned}
\alpha((\gamma H, z), g) & :=\left(\gamma,\left(z, \sigma(z)(\bar{s}(\gamma))^{-1} \cdot g\right)\right), \\
\beta(\gamma,(z, g)) & :=((\gamma H, z), \sigma(z)(\bar{s}(\gamma)) \cdot g) .
\end{aligned}
$$

Then $\alpha$ and $\beta$ are to one another inverse $G$-homeomorphisms. They induce isomorphisms of principal $G$-bundles from $p_{\sigma}$ restricted to $U \times Z$ to the trivial principal $G$ bundle over $U \times Z$. One easily checks using Lemma A.1(ii) applied to the standard map $\Gamma / H \times Z=k\left(\Gamma / H \times_{p} Z\right) \rightarrow \Gamma / H \times_{p} Z$ (see Sections A.2 and A.3) and Lemma A.1(i) that $U \times Z$ is a quasiregular open subset of $\Gamma / H \times Z$. Since $\gamma H \in \Gamma / H$ is contained in the open subset $\gamma \cdot U$ and $p_{\sigma}$ is a $\Gamma$-equivariant map, we conclude that $p_{\sigma}$ is locally trivial and hence a principal $G$-bundle.

(ii) In the sequel we identify the subspace $\{1\} \times Z$ of $\Gamma / H \times Z$ with $Z$. Define the $\Gamma-$ map

$$
u: \Gamma \times_{H} p^{-1}(Z) \rightarrow E, \quad(\gamma, e) \rightarrow \gamma \cdot e .
$$

It is a homeomorphism by Lemma 4.2. It is compatible with the natural right $G$-actions and commutes with the left $\Gamma$-actions. The restriction $\left.p\right|_{p^{-1}(Z)}: p^{-1}(Z) \rightarrow Z$ of $p$ to $Z=\{1\} \times Z$ is a principal $G$-bundle over $Z$. By assumption $\left.p\right|_{p^{-1}(Z)}$ is isomorphic to the trivial principal $G$-bundle over $Z$. Hence we can choose a $G$-homeomorphism

$$
f: Z \times G \stackrel{\cong}{\longrightarrow} p^{-1}(Z)
$$


such that the composite $\left.p\right|_{p^{-1}(Z)} \circ f$ is the canonical projection $Z \times G \rightarrow Z$. Let $\sigma: Z \rightarrow \operatorname{hom}(H, G)$ be defined by the map $\rho_{f(z, 1)}$ (see (3-1)), ie $\sigma$ is uniquely determined by $h \cdot f(z, 1)=f(z, 1) \cdot \sigma(z)(h)$. (It is continuous because of the properties of the category of compactly generated spaces listed in Section A.4.) We define a left $H$-action on $Z \times G$ by $h \cdot(z, g)=(z, \sigma(z)(h) \cdot g)$. Then $f$ is compatible with the left $H$-actions by the calculation

$$
\begin{aligned}
f(h \cdot(z, g)) & =f(z, \sigma(z)(h) \cdot g) \\
& =f(z, 1) \cdot \sigma(z)(h) \cdot g=h \cdot f(z, 1) \cdot g=h \cdot f(z, g) .
\end{aligned}
$$

We obtain a homeomorphism compatible with the obvious left $\Gamma$-actions and with the obvious right $G$-actions

$$
\Gamma \times_{H}(Z \times G) \stackrel{\operatorname{id}_{\Gamma} \times_{H} f}{\longrightarrow} \Gamma \times \times_{H} p^{-1}(Z) \stackrel{u}{\rightarrow} E .
$$

This is an isomorphism of $\Gamma$-equivariant principal $G$-bundles from $p_{\sigma}: \Gamma \times{ }_{H}(Z \times G) \rightarrow$ $\Gamma / H \times Z$ to $p: E \rightarrow \Gamma / H \times Z$.

(iii) Let

$$
f: \Gamma \times_{H}(Z \times G) \stackrel{\cong}{\longrightarrow} \Gamma \times_{H}(Z \times G)
$$

be an isomorphism of $\Gamma$-equivariant principal $G$-bundles from $p_{\sigma_{0}}$ to $p_{\sigma_{1}}$. Notice that the left $H$-actions on $(Z \times G)$ in the source and the target are different, the first one depends on $\sigma_{0}$, the second on $\sigma_{1}$. There is precisely one map $\omega: Z \rightarrow G$ satisfying $f(1,(z, 1))=(1,(z, \omega(z)))$. (It is continuous because of the properties of the category of compactly generated spaces listed in Section A.4.) Since $f$ is compatible with the natural left $\Gamma$-actions and with the natural right $G$-actions, we get for $h \in H$ and $z \in Z$,

$$
\begin{aligned}
\left(1,\left(z, \sigma_{1}(z)(h) \cdot \omega(z)\right)\right) & =(1, h \cdot(z, \omega(z)))=(h,(z, \omega(z))) \\
& =h \cdot(1,(z, \omega(z)))=h \cdot f(1,(z, 1)) \\
& =f(h \cdot(1,(z, 1)))=f(h,(1, z)) \\
& =f(1, h \cdot(z, 1))=f\left(1,\left(z, \sigma_{0}(z)(h)\right)\right. \\
& =f(1,(z, 1)) \cdot \sigma_{0}(z)(h)=(1, z, \omega(z)) \cdot \sigma_{0}(z)(h) \\
& =\left(1,\left(z, \omega(z) \cdot \sigma_{0}(z)(h)\right)\right) .
\end{aligned}
$$

This implies $\sigma_{1}(z)(h) \cdot \omega(z)=\omega(z) \cdot \sigma_{0}(z)(h)$ for all $h \in H$ and $z \in Z$.

For the converse, the map

$$
\begin{aligned}
\Gamma \times_{H}(Z \times G) & \rightarrow \Gamma \times_{H}(Z \times G), \\
(\gamma,(z, g)) & \mapsto(\gamma,(z, w(z) g)))
\end{aligned}
$$


satisfies the desired properties.

(iv) Consider $(\gamma,(z, g)) \in \Gamma \times \times_{H}(Z \times G)$. One easily checks

$$
\Gamma_{(\gamma H, z)}=\gamma \cdot \Gamma_{(1 H, z)} \cdot \gamma^{-1}=\gamma \cdot H \cdot \gamma^{-1} .
$$

We compute for $h \in H$ in $\Gamma \times{ }_{H}(Z \times G)$,

$$
\begin{aligned}
\left(\gamma \cdot h \cdot \gamma^{-1}\right) \cdot(\gamma,(z, g))=(\gamma \cdot h,(z, g)) & =(\gamma, h \cdot(z, g)) \\
& =(\gamma,(z, \sigma(z)(h) \cdot g)) \\
& =\left(\gamma,\left(z, g \cdot\left(g^{-1} \cdot \sigma(z)(h) \cdot g\right)\right)\right) \\
& =(\gamma,(z, g)) \cdot\left(g^{-1} \cdot \sigma(z)(h) \cdot g\right) .
\end{aligned}
$$

This finishes the proof of Lemma 5.1.

\section{Discussion of homotopy invariance and Condition (H)}

Since we want to have a bundle theory for which there exists a universal bundle, we have to ensure homotopy invariance, ie if $f_{0}, f_{1}: B_{0} \rightarrow B_{1}$ are $\Gamma$-maps and $p: E \rightarrow B_{1}$ is a $\Gamma$-equivariant principal $G$-bundle, we want to arrange that the pullbacks $f_{0}^{*} p$ and $f_{1}^{*} p$ are isomorphic as $\Gamma$-equivariant principal $G$-bundles.

Let $Z$ be a contractible CW-complex and $H \subseteq \Gamma$ be a subgroup. Equip $Z$ with the trivial $\Gamma$-action. Then the projection pr: $\Gamma / H \times Z \rightarrow \Gamma / H$ is a $\Gamma$-homotopy equivalence. Hence in order to guarantee homotopy invariance, we must ensure that every $\Gamma$-equivariant principal $G$-bundle $p: E \rightarrow \Gamma / H \times Z$ over $\Gamma / H \times Z$ is isomorphic to $\operatorname{pr}^{*} E^{\prime}$ for some $\Gamma$-equivariant principal $G$-bundle on $p^{\prime}: E^{\prime} \rightarrow \Gamma / H$ over $\Gamma / H$.

For $\alpha \in \operatorname{hom}(H, G)$ the centralizer of $\alpha$ in $G$ is defined to be the subgroup of $G$ given by

$$
C_{G}(\alpha):=\left\{g \in G \mid g \alpha(h) g^{-1}=\alpha(h) \text { for all } h \in H\right\} .
$$

Definition 6.1 (Condition $(\mathrm{H})$ ) A family $\mathcal{R}$ of local representations in the sense of Definition 3.1 satisfies Condition $(\mathrm{H})$ if the following hold for every $(H, \alpha) \in \mathcal{R}$ :

(i) The path component of $\alpha$ in $\operatorname{hom}(H, G)$ is contained in $\left\{c_{g} \circ \alpha \mid g \in G\right\}$.

(ii) The pair $\left(G, C_{G}(\alpha)\right)$ satisfies Condition (S) introduced in Definition 4.1.

(iii) The pair $(\Gamma, H)$ satisfies Condition (S) introduced in Definition 4.1.

(iv) The canonical map

$$
\iota_{\alpha}: G / C_{G}(\alpha) \rightarrow \operatorname{hom}(H, G), \quad g C_{G}(\alpha) \mapsto c_{g} \circ \alpha
$$

is a homeomorphism onto its image. 
Lemma 6.2 Suppose that the family $\mathcal{R}$ of local representations satisfies Condition $(H)$. Let $Z$ be a (nonequivariant) contractible $C W$-complex and let $p: E \rightarrow \Gamma / H \times Z$ be a $\Gamma$-equivariant principal $G$-bundle with $\mathcal{R}(p) \subseteq \mathcal{R}$.

Then $p$ is isomorphic to $\mathrm{pr}^{*} E^{\prime}$ for a $\Gamma$-equivariant principal $G$-bundle $p^{\prime}: E^{\prime} \rightarrow \Gamma / H$ for the projection pr: $\Gamma / H \times Z \rightarrow \Gamma / H$, or, equivalently, there exists an element $(H, \alpha)$ in $\mathcal{R}$ such that $p$ is isomorphic to the $\Gamma$-equivariant principal bundle

$$
p_{\alpha}:\left(\Gamma \times_{H} G\right) \times Z \rightarrow \Gamma / H \times Z, \quad((\gamma, g), z) \mapsto(\gamma H, z) .
$$

Proof Since $Z$ is contractible, by Lemma 1.3, we have that the restriction bundle

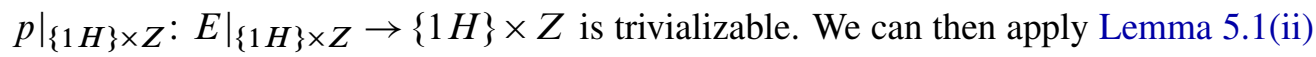
and we obtain that $p$ is isomorphic to $p_{\sigma}$ for an appropriate map $\sigma: Z \rightarrow \operatorname{hom}(H, G)$. Since $Z$ is path connected, the image of $\sigma$ is contained in a path component of $\alpha \in \operatorname{hom}(H, G)$ if we take $\alpha=\sigma(z)$ for some $z \in Z$. Lemma 5.1(iv) implies that $(H, \alpha)$ belongs to $\mathcal{R}(p)$ and hence to $\mathcal{R}$. Because of Condition $(\mathrm{H})$ the image of $\sigma: Z \rightarrow \operatorname{hom}(H, G)$ is contained in the image of $\iota_{\alpha}: G / C_{G}(\alpha) \rightarrow \operatorname{hom}(H, G)$. Since $\iota_{\alpha}$ is a homeomorphism onto its image by Condition $(\mathrm{H})$, we can find a map $\bar{\omega}: Z \rightarrow G / C_{G}(\alpha)$ with $\iota_{\alpha} \circ \bar{\omega}=\sigma$. Because of Condition (H) and Remark 4.3, the projection $G \rightarrow G / C_{G}(\alpha)$ is a principal $C_{G}(\alpha)$-bundle. Hence its pullback with $\bar{\omega}$ is a principal $C_{G}(\alpha)$-bundle over the contractible CW-complex $Z$ and hence has a section by Lemma 1.3. Thus we can find a map $\omega: Z \rightarrow G$ whose composite with the projection $G \rightarrow G / C_{G}(\alpha)$ is $\bar{\omega}$. This implies that $\sigma=c_{\omega} \circ \alpha$. Now apply Lemma 5.1(iii).

Theorem 6.3 Let $\mathcal{R}$ be a family of local representations. Then it satisfies Condition $(H)$ if the following conditions are satisfied.

(i) The group $\Gamma$ is locally compact, second countable and has finite covering dimension, eg is a Lie group, or $\Gamma$ is completely regular and all compact subgroups of $\Gamma$ are Lie groups, eg is a Kac-Moody group.

(ii) The group $G$ is locally compact, second countable and has finite covering dimension, eg is a Lie group.

(iii) The group $G$ is almost connected (see Definition 12.3).

(iv) For every element $(H, \alpha)$ the group $H$ is a compact group.

Proof Condition (i) appearing in Definition 6.1 is proved in Theorem B.1.

Conditions (ii) and (iii) appearing in Definition 6.1 follow from Lemma 4.4(ii).

Condition (iv) appearing in Definition 6.1 is proved in Theorem B.2. 


\section{The slice theorem for equivariant $\mathrm{CW}$-complexes}

In this section we prove the following slice theorem for $\Gamma-\mathrm{CW}$-complexes, generalizing [22, Theorem 1.37].

Theorem 7.1 (Slice theorem) Let $G$ be a (compactly generated) topological group and let $X$ be a $G-C W$-complex. Consider $x \in X$ together with a $G_{X}$-invariant neighborhood $V_{x}$. Suppose that the pair $\left(G, G_{x}\right)$ satisfies Condition (S); see Definition 4.1. Then there exists a $G_{x}$-invariant subset $S_{x}$ of $x$ with the following properties:

(i) $S_{x}$ inherited with the subspace topology is compactly generated.

(ii) The closure of $S_{x}$ is contained in $V_{x}$.

(iii) The inclusion $\{x\} \rightarrow S_{x}$ is a $G_{x}$-homotopy equivalence.

(iv) The set $U:=G \cdot S_{x}$ is a quasiregular $G$-invariant open subset of $X$ and the map

$$
G \times_{G_{x}} S_{x} \cong U, \quad(g, s) \mapsto g \cdot s
$$

is a $G$-homeomorphism.

Proof Let $n_{x} \geq 0$ be the integer for which $x \in X_{n_{x}}$ and $x \notin X_{n_{x}-1}$. We construct inductively for $n=n_{x}, n_{x}+1, \ldots$ open $G$-invariant subsets $U[n] \subseteq X$ such that the following conditions hold if we put $V=G \cdot V_{x}$.

(1) We have $U[n] \subseteq X_{n}, U[n] \subseteq U[n+1], \overline{U[n]} \subseteq V$ and $x \in U[n]$ for all $n \geq n_{x}$.

(2) For each $n \geq n_{x}$ there is a $G$-map

$$
r[n+1]: U[n+1] \rightarrow U[n]
$$

satisfying $r[n+1] \circ i[n+1]=\mathrm{id}_{U[n]}$, where $i[n+1]: U[n] \rightarrow U[n+1]$ is the inclusion.

(3) For each $n \geq n_{x}$ there is a $G$-homotopy

$$
h[n+1]: U[n+1] \times[0,1] \rightarrow U[n+1]
$$

satisfying

$$
\begin{aligned}
h[n+1]_{t} & =\operatorname{id}_{U[n+1]} & & \text { for } 0 \leq t \leq(n+3)^{-1}, \\
h[n+1]_{t} & =i[n+1] \circ r[n+1] & & \text { for }(n+2)^{-1} \leq t \leq 1, \\
h[n+1](z, t) & =z & & \text { for } z \in U[n], t \in[0,1], \\
r[n+1] \circ h[n+1] & =r[n+1] \circ \operatorname{pr}_{n+1}, & &
\end{aligned}
$$

where $\operatorname{pr}_{n+1}: U[n+1] \times[0,1] \rightarrow U[n+1]$ is the projection. 
(4) There is a $G$-map

$$
r\left[n_{x}\right]: U\left[n_{x}\right] \rightarrow G x
$$

so $r\left[n_{x}\right] \circ i\left[n_{x}\right]=\mathrm{id}_{G x}$ holds, where $G x:=\{g x \mid g \in G\}$ and $i\left[n_{x}\right]: G x \rightarrow U\left[n_{x}\right]$ is the inclusion.

(5) There is a $G$-homotopy

$$
h\left[n_{x}\right]: U\left[n_{x}\right] \times[0,1] \rightarrow U\left[n_{x}\right]
$$

satisfying

$$
\begin{aligned}
h\left[n_{x}\right]_{t} & =\operatorname{id}_{U_{n_{x}}} & & \text { for } t \leq\left(n_{x}+2\right)^{-1}, \\
h\left[n_{x}\right]_{t} & =i\left[n_{x}\right] \circ r\left[n_{x}\right] & & \text { for } t \geq\left(n_{x}+1\right)^{-1}, \\
h\left[n_{x}\right](g x, t) & =g x & & \text { for } g x \in G x, t \in[0,1], \\
r\left[n_{x}\right] \circ h\left[n_{x}\right] & =r\left[n_{x}\right] \circ \operatorname{pr}_{n_{x}}, & &
\end{aligned}
$$

where $\operatorname{pr}_{n_{x}}: U\left[n_{x}\right] \times[0,1] \rightarrow U\left[n_{x}\right]$ is the projection.

Recall that we have for each $n \geq 0$ a $G$-pushout of the form

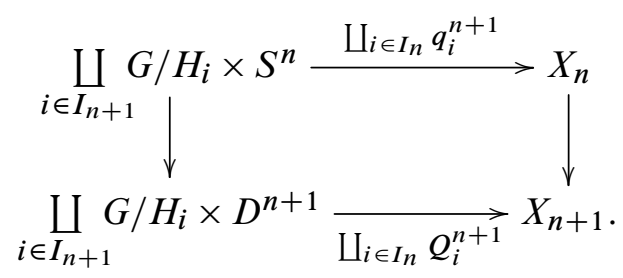

Next we explain the beginning of the induction. Since $x$ belongs to $X_{n_{x}}$ but not to $X_{n_{x}-1}$, we can find $i \in I_{n_{x}}$ and $\left(\gamma H_{i}, y\right) \in G / H_{i} \times\left(D^{n_{x}} \backslash S^{n_{x}-1}\right)$ satisfying $Q_{i}^{n_{x}}\left(g H_{i}, y\right)=x$. Choose $\delta_{i}>0$ such that $G / H_{i} \times \overline{B_{\delta}(y)}$ is contained in both $G / H_{i} \times D^{n_{x}} \backslash S^{n_{x}-1}$ and $\left(Q_{i}^{n_{x}}\right)^{-1}\left(V_{x} \cap X_{n_{x}}\right)$, where $B_{\delta}(y)$ is the ball of radius $\delta$ around $y$. Define a $G-$ map

$$
r\left[n_{x}\right]^{\prime}: G / H_{i} \times B_{\delta}(y) \rightarrow G y, \quad\left(g H_{i}, z\right) \mapsto g y
$$

and a $G$-homotopy

$$
h\left[n_{x}\right]^{\prime}: G / H_{i} \times B_{\delta}(y) \times[0,1] \rightarrow G / H_{i} \times B_{\delta}(y)
$$


by sending $\left(g H_{i}, z, t\right)$ to

$\left(g H_{i}, z\right)$ for $t \leq\left(n_{x}+2\right)^{-1}$,

$\left(g H_{i}, \frac{t-\left(n_{x}+2\right)^{-1}}{\left(n_{x}+1\right)^{-1}-\left(n_{x}+2\right)^{-1}} \cdot y+\left(1-\frac{t-\left(n_{x}+2\right)^{-1}}{\left(n_{x}+1\right)^{-1}-\left(n_{x}+2\right)^{-1}}\right) \cdot z\right)$ for $\left(n_{x}+2\right)^{-1} \leq t \leq\left(n_{x}+1\right)^{-1}$,

$\left(g H_{i}, y\right)$ for $t \geq\left(n_{x}+1\right)^{-1}$.

Now define

$$
U\left[n_{x}\right]=Q_{i}^{n_{x}}\left(G / H_{i} \times B_{\delta}(y)\right) .
$$

Let

$$
h\left[n_{x}\right]: U\left[n_{x}\right] \times[0,1] \rightarrow U\left[n_{x}\right]
$$

be the $G$-homotopy uniquely determined by the property that for every element $\left(g H_{i}, z, t\right) \in G / H_{i} \times B_{\delta}(y) \times[0,1], Q_{i}^{n_{x}} \circ h\left[n_{x}\right]^{\prime}\left(g H_{i}, z, t\right)=h\left[n_{x}\right]\left(Q_{i}^{n_{x}}\left(g H_{i}, z\right), t\right)$ holds. Define the $G$-map

$$
r\left[n_{x}\right]: U\left[n_{x}\right] \rightarrow G x
$$

analogously using $r\left[n_{x}\right]^{\prime}$. One easily checks that $U\left[n_{x}\right], r\left[n_{x}\right]$ and $h\left[n_{x}\right]$ have the desired properties.

Next we explain the induction step from $n \geq n_{x}$ to $n+1$. For a real number $\epsilon \in(0,1)$ define the subspace

$$
S^{n}[\epsilon]:=\left\{t \cdot z \mid t \in(1-\epsilon, 1], z \in S^{n}\right\} \subseteq D^{n+1},
$$

a map

$$
p[\epsilon]: S^{n}[\epsilon] \rightarrow S^{n}, \quad t \cdot z \mapsto z
$$

and a homotopy

$$
l[\epsilon]: S^{n}[\epsilon] \times[0,1] \rightarrow S^{n}[\epsilon], \quad(t \cdot z, s) \mapsto(t \cdot(1-s)+s) \cdot z .
$$

If $j[\epsilon]: S^{n} \rightarrow S^{n}[\epsilon]$ is the inclusion, then $l[\epsilon]_{0}=\operatorname{id}_{S^{n}[\epsilon]}$ and $l[\epsilon]_{1}=i[\epsilon] \circ p[\epsilon]$. Roughly speaking, the homotopy $l[\epsilon]$ pushes $S^{n}[\epsilon]$ radially to $S^{n}$.

Roughly speaking, we will obtain $U[n+1]$ from $U[n]$ by a thickening into the interior of the various equivariant $(n+1)$-cells such that the thickening is small enough to ensure that the closure of $U[n+1]$ stays within $V$. In detail, consider $i \in I_{n+1}$. Then we get inclusions of $G$-invariants subsets of $\Gamma / H_{i} \times S^{n}$

$$
\left(q_{i}^{n+1}\right)^{-1}(U[n]) \subseteq\left(q_{i}^{n+1}\right)^{-1}(\overline{U[n]}) \subseteq\left(q_{i}^{n+1}\right)^{-1}(V)
$$


because of the induction hypothesis. Notice that $\left(Q_{i}^{n+1}\right)^{-1}(V)$ is a $G$-invariant open subset of $G / H_{i} \times D^{n+1}$ containing the closed $G$-invariant subset $\left(q_{i}^{n+1}\right)^{-1}(\overline{U[n]})$. Let pr: $G / H_{i} \times D^{n+1} \rightarrow D^{n+1}$ be the projection. Then the set $\operatorname{pr}\left(\left(Q_{i}^{n+1}\right)^{-1}(V)\right)$ is open in $D^{n+1}$ and contains the closed subset $\operatorname{pr}\left(\left(q_{i}^{n+1}\right)^{-1}(\overline{U[n]})\right)$. Since $S^{n}$ is compact, we can choose $\epsilon_{i}>0$ such that

$$
p\left[2 \cdot \epsilon_{i}\right]^{-1}\left(\operatorname{pr}\left(\left(q_{i}^{n+1}\right)^{-1}(\overline{U[n]})\right)\right) \subseteq \operatorname{pr}\left(\left(Q_{i}^{n+1}\right)^{-1}(V)\right)
$$

holds. Define

$$
U[n+1]:=U[n] \cup \bigcup_{i \in I_{n+1}} Q_{i}^{n+1}\left(\operatorname{pr}^{-1}\left(p\left[\epsilon_{i}\right]^{-1}\left(\operatorname{pr}\left(\left(q_{i}^{n+1}\right)^{-1}(\overline{U[n]})\right)\right)\right)\right) .
$$

One easily checks that $U[n+1]$ is an open $G$-invariant subset of $X_{n+1}$ such that $\overline{U[n+1]} \subseteq V$ holds. The various $G-\operatorname{maps}_{G / H_{i}} \times p\left[\epsilon_{i}\right]: G / H_{i} \times S^{n}[\epsilon] \rightarrow G / H_{i} \times S^{n}$ fit together to a $G$-map

$$
r[n+1]: U[n+1] \times[0,1] \rightarrow U[n+1]
$$

such that $r[n+1] \circ i[n+1]=\mathrm{id}_{U[n]}$ holds for the inclusion $i[n+1]: U[n] \rightarrow U[n+1 \mid$. The various $G$-homotopies $\operatorname{id}_{G / H_{i}} \times l\left[\epsilon_{i}\right]: G / H_{i} \times S^{n}[\epsilon] \rightarrow G / H_{i} \times S^{n}$ fit together to a $G$-homotopy

$$
h[n+1]^{\prime}: U[n+1] \times[0,1] \rightarrow U[n+1]
$$

such that $h[n+1]_{0}^{\prime}=\operatorname{id}_{U[n+1]}$, the image of $h[n+1]_{1}$ lies in $U[n+1] \cap X_{n}=U[n]$, the restriction of $h[n+1]_{t}^{\prime}: U[n+1] \rightarrow U[n+1]$ to $U[n]$ is the inclusion $U[n] \rightarrow U[n+1]$ for all $t \in[0,1]$ and $r[n+1] \circ h[n+1]=r[n+1] \circ \operatorname{pr}_{n+1}$ for $\operatorname{pr}_{n+1}: U[n+1] \times[0,1] \rightarrow$ $U[n+1]$. Define a map

$$
\tau:[0,1] \rightarrow[0,1], \quad t \mapsto \begin{cases}0 & 0 \leq t \leq(n+3)^{-1}, \\ \frac{t-(n+3)^{-1}}{(n+2)^{-1}-(n+3)^{-1}} & (n+3)^{-1} \leq t \leq(n+2)^{-1}, \\ 1 & (n+2)^{-1} \leq t .\end{cases}
$$

One easily checks that the $G$-homotopy

$$
h[n+1]:=h[n+1]^{\prime} \circ\left(\operatorname{id}_{U[n+1]} \times \tau\right): U[n+1] \times[0,1] \rightarrow U[n+1]
$$

has the desired properties. This finishes the induction step.

Now define

$$
U:=\bigcup_{n \geq n_{x}} U[n]
$$

Since each $U[n] \subseteq X_{n}$ is an open $G$-invariant subset of $X_{n}$ and $X$ has the weak topology with respect to the filtration by its skeletons $\left\{X_{n} \mid n \geq-1\right\}$, the subset $U$ 
of $X$ is open and $G$-invariant. Since $\bar{U}_{n} \subseteq V$ holds for all $n \geq 0$, we conclude $\bar{U} \subseteq V$. Define the $G$-map

$$
\rho[n]:=U[n] \rightarrow G x
$$

to be the composite $U[n] \stackrel{r[n]}{\longrightarrow} U[n-1] \stackrel{r[n-1]}{\longrightarrow} \cdots \stackrel{r\left[n_{x}+1\right]}{\longrightarrow} U\left[n_{x}\right] \stackrel{r\left[n_{x}\right]}{\longrightarrow} G x$. Since $\rho[n+1]$ restricted to $U[n]$ is $\rho[n]$, we obtain a $G-$ map

$$
\rho: U \rightarrow G x
$$

Let $\iota: G x \rightarrow U$ be the inclusion. Obviously $\rho \circ \iota=\mathrm{id}_{G x}$. Next we define inductively for $n=n_{x}, n_{x}+1, \ldots G$-homotopies

$$
k[n]: U[n] \times[0,1] \rightarrow U[n]
$$

such that $k[n]_{t}=\mathrm{id}_{U[n]}$ holds for $t \in\left[0,(n+2)^{-1}\right]$, we have $k[n]_{1}=\rho[n]$, the composite $\rho[n] \circ k[n]: U[n] \times[0,1] \rightarrow G x$ factorizes over the projection $U[n] \times[0,1] \rightarrow U[n]$ to $\rho[n]: U[n] \rightarrow G x$, and the following diagram commutes:

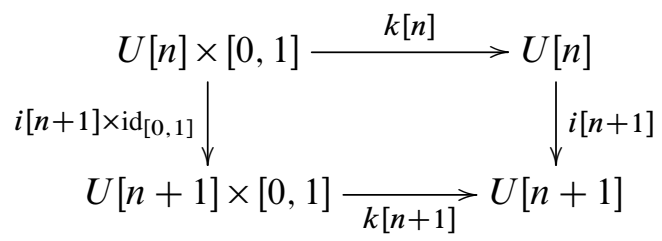

In the induction beginning put $k\left[n_{x}\right]=h\left[n_{x}\right]$. In the induction step from $n \geq n_{x}$ to $n+1$, we define $\left.k[n+1]\right|_{U[n+1] \times\left[0,(n+2)^{-1}\right]}$ to be $\left.h[n+1]\right|_{U[n+1] \times\left[0,(n+2)^{-1}\right]}$, and define $\left.k[n+1]\right|_{U[n+1] \times\left[(n+2)^{-1}, 1\right]}$ to be the composite

$$
\begin{aligned}
U[n+1] \times\left[(n+2)^{-1}, 1\right] \stackrel{r[n+1] \times \operatorname{id}_{\left[(n+2)^{-1}, 1\right]}}{\longrightarrow} U[n] \times\left[(n+2)^{-1}, 1\right] \\
\stackrel{\left.k[n]\right|_{U[n] \times\left[(n+2)^{-1}, 1\right]}}{\longrightarrow} U[n] \stackrel{i[n+1]}{\longrightarrow} U[n+1] .
\end{aligned}
$$

The homotopies $k[n]$ for $n=n_{x}, n_{x}+1, \ldots$ fit together to a $G$-homotopy

$$
k: U \times[0,1] \rightarrow U
$$

with $k_{0}=\mathrm{id}_{U}$ and $k_{1}=\iota \circ \rho$. To summarize, we have $\rho \circ \iota=\mathrm{id}_{G x}$ and $k: \iota \circ \rho \simeq_{G} \operatorname{id}_{U}$, and the composite $\rho \circ k: U \times[0,1] \rightarrow G x$ factorizes over the projection $U \times[0,1] \rightarrow U[n]$ to $\rho$. 
Let $S_{x}$ be the preimage of $x$ under the map $\rho: U \rightarrow G x$. The $G$-map $G / G_{x} \rightarrow G x$, $g \mapsto g x$ is obviously a $G$-homeomorphism. Since the pair $\left(G, G_{x}\right)$ satisfies Condition (S) by assumption, Lemma 4.2 implies that the map

$$
u: G \times_{G_{x}} S_{x} \cong U, \quad(g, s) \mapsto g s
$$

is a $G$-homeomorphism. The restriction of $\iota$ to $\{x\}$ is the inclusion $i:\{x\} \rightarrow S_{x}$. The restriction of $\rho$ to $S_{x}$ is a $G_{x}$-homotopy inverse of $i$ since $\rho \circ k: U \times[0,1] \rightarrow G x$ factorizes over the projection $U \times[0,1] \rightarrow U[n]$ to $\rho$.

Since $V$ is by definition $G \cdot V_{x}$ and the closure of $U$ is contained in $V$, the closure of $S_{x}$ is contained in $V_{x}$.

We conclude from Lemma A.1(vi) that $U=\operatorname{pr}^{-1}(\operatorname{pr}(U))$ is quasiregular and hence $U$ equipped with the subspace topology is compactly generated. Since $S_{x} \subseteq U$ is closed, also $S_{x}$ is compactly generated. This finishes the proof of Theorem 7.1.

\section{Equivariant principal bundles versus equivariant CW- complexes}

In this section we prove one of our main technical results saying that a $\Gamma$-equivariant principal $G$-bundle $p: E \rightarrow B$ is the same as a $\Gamma \times G-\mathrm{CW}$-complex $E$ with a special structure of its $\Gamma \times G$-isotropy groups provided that $\mathcal{R}(p) \subseteq \mathcal{R}$ holds for a given family of local representations $\mathcal{R}$ satisfying Condition $(\mathrm{H})$.

Theorem 8.1 Let $\mathcal{R}$ be a family of local representations for $(\Gamma, G)$ satisfying Condition $(H)$ introduced in Definition 6.1.

(i) Let $p: E \rightarrow B$ be a $\Gamma$-equivariant principal $G$-bundle with $\mathcal{R}(p) \subseteq \mathcal{R}$ over a $\Gamma-C W$-complex $B$. Then $E_{l}$ is a $\Gamma \times G-C W$-complex whose isotropy groups belong to the family $\mathcal{F}(\mathcal{R})$ introduced in Lemma 3.5.

(ii) Let $E$ be a left $\Gamma \times G-C W$-complex whose isotropy groups belong to $\mathcal{F}(\mathcal{R})$. Then $p: E_{r} \rightarrow E_{r} / G$ is a $\Gamma$-equivariant principal $G$-bundle with $\mathcal{R}(p) \subseteq \mathcal{R}$.

Proof (i) Let $B_{n}$ be the $n-$ skeleton of the $\Gamma-\mathrm{CW}-$ complex structure on $B$. Put $E_{n}:=p^{-1}\left(B_{n}\right)$. Let $p_{n}: E_{n} \rightarrow B_{n}$ be the $\Gamma$-equivariant principal $G$-bundle obtained by restricting $p$ to $B_{n}$. Next we will show that the filtration of $E$ by the $E_{n}-$ s induces the structure of a $\Gamma \times G-\mathrm{CW}$-structure on $E_{l}$ whose isotropy groups belong to $\mathcal{F}(\mathcal{R})$. 
Fix $n \geq 0$. Since $B$ is a $\Gamma-\mathrm{CW}-$ complex, there exists a $\Gamma$-pushout

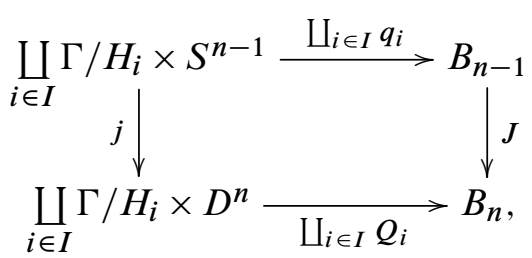

where $j$ and $J$ are the inclusions. Consider the square obtained by the pullback construction applied to $p_{n}: E_{n} \rightarrow B_{n}$ :

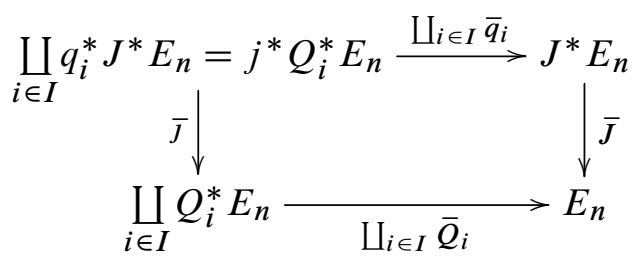

This is a diagram of $\Gamma \times G$-spaces, the various left $\Gamma \times G$-actions come from the left $\Gamma \times G$-action on $E_{l}$. It is a $\Gamma \times G$-pushout by Lemma A.5(i) and (ii).

We conclude from Remark 3.2 that $\mathcal{R}\left(Q_{i}^{*} E_{n}\right) \subseteq \mathcal{R}$ holds. Lemma 6.2 implies that the $\Gamma$-equivariant principal $G$-bundle $Q_{i}^{*} E_{n}$ is isomorphic to the $\Gamma$-equivariant principal $G$-bundle $p_{\alpha}:\left(\Gamma \times{ }_{H} G\right) \times D^{n} \rightarrow \Gamma / H \times D^{n}$ for some element $(H, \alpha) \in \mathcal{R}$. Hence there exists a $\Gamma \times G$-homeomorphism of left $\Gamma \times G$-pairs

$$
(\Gamma \times G) / H_{i}^{\prime} \times\left(D^{n}, S^{n-1}\right) \stackrel{\cong}{\longrightarrow}\left(\left(Q_{i}^{*} E_{n}\right)_{l},\left(q_{i}^{*} J^{*} E_{n}\right)_{l}\right)
$$

for an appropriate subgroup $H_{i}^{\prime} \subseteq \Gamma \times G$ belonging to $\mathcal{F}(\mathcal{R})$.

It remains to show that $E$ has the weak topology with respect to the filtration given by the $E_{n}-$ s. This follows from Lemma A.6(i) and (ii). This finishes the proof of assertion (i).

(ii) Let $E$ be a left $\Gamma \times G-\mathrm{CW}$-complex whose isotropy groups belong to $\mathcal{F}(\mathcal{R})$. Firstly, we show that $E / G$ is a $\Gamma-\mathrm{CW}$-complex. Consider $e \in E$. There exists $(H, \alpha) \in \mathcal{R}$ such that $(\Gamma \times G)_{e}=K(H, \alpha)=\{(h, \alpha(h) \mid h \in H\}$. Hence the image of $(\Gamma \times G)_{e}$ under the projection $\Gamma \times G \rightarrow \Gamma$ is $H$ and hence closed. Lemma A.7 implies that $E / G$ is a $\Gamma-\mathrm{CW}-$ complex.

Next we show that $p: E_{r} \rightarrow E / G$ is a principal $G$-bundle. Consider $b \in E / G$. Choose $e \in E$ with $p(e)=b$. From the Slice Theorem 7.1 we obtain a $(\Gamma \times G)_{e}-$ invariant (compactly generated) subspace $S$ of $E$ containing $e$ such that the map

$$
f:(\Gamma \times G) \times(\Gamma \times G)_{e} S \rightarrow(\Gamma \times G) \cdot S, \quad((\gamma, g), s) \mapsto(\gamma, g) \cdot s
$$


is a $(\Gamma \times G)$-homeomorphism and $(\Gamma \times G) \cdot S$ is an open $\Gamma \times G$-invariant (compactly generated) subset of $E$. Put $V:=p((\Gamma \times G) \cdot S)$. Choose $(H, \alpha) \in \mathcal{R}$ such that

$$
(\Gamma \times G)_{e}=K(H, \alpha)=\{(h, \alpha(h) \mid h \in H\} .
$$

We equip $S$ with the left $H$-action given by $h \cdot s:=(h, \alpha(h)) \cdot s$. We obtain a $\Gamma$-map

$$
u: \Gamma \times_{H} S \rightarrow V, \quad(\gamma, s) \rightarrow p \circ f((\gamma, e), s) .
$$

Define the $\Gamma \times G-$ map

$$
q:(\Gamma \times G) \times(\Gamma \times G)_{e} S \rightarrow \Gamma \times_{H} S, \quad((\gamma, g), s) \mapsto(\gamma, s),
$$

where $G$ acts trivially on the target. Then $V$ is an open $\Gamma$-invariant neighborhood of $b$ in $E / G$ which is quasiregular by Lemma A.1(vi). Moreover, the following diagram of right $G$-spaces commutes, where $G$ acts trivial on $\Gamma{ }{ }_{H} S$ and $V$, has a $G$-homeomorphism as upper horizontal map and identifications as vertical maps:

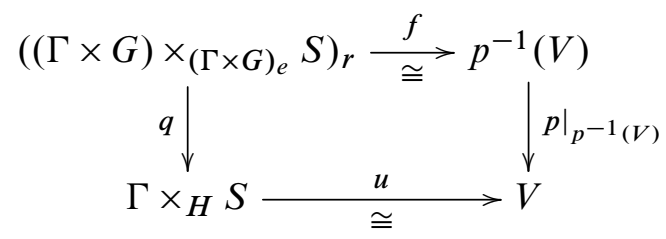

Since $u$ is a bijective identification, it is a homeomorphisms. Hence it suffices to show that $q$ is a principal $G$-bundle.

Equip $S \times G$ with the left $H$-action given by $h \cdot(s, g):=(h \cdot s, \alpha(h) \cdot g)$. Then

$$
p_{\alpha}: \Gamma \times_{H}(S \times G) \rightarrow \Gamma \times_{H} S, \quad(\gamma,(s, g)) \mapsto(\gamma, s)
$$

is a well-defined map which is compatible with the obvious left $\Gamma$-actions on the source and the target and with the obvious right $G$-action on the source and the trivial right $G$-action on the target. Define a map

$$
\xi: \Gamma \times_{H}(S \times G) \stackrel{\cong}{\rightarrow}\left((\Gamma \times G) \times(\Gamma \times G)_{e} S\right)_{r}, \quad(\gamma,(s, g)) \mapsto\left(\left(\gamma, g^{-1}\right), s\right) .
$$

It is well-defined by the following calculation for $\gamma \in \Gamma, s \in S$ and $g \in G$ :

$$
\begin{aligned}
\left(\left(\gamma h^{-1},(\alpha(h) \cdot g)^{-1}\right), h \cdot s\right) & =\left(\left(\gamma \cdot h^{-1}, g^{-1} \alpha(h)^{-1}\right), h \cdot s\right) \\
& =\left(\left(\gamma, g^{-1}\right) \cdot(h, \alpha(h))^{-1},(h, \alpha(h)) \cdot s\right)=\left(\left(\gamma, g^{-1}\right), s\right)
\end{aligned}
$$

The map $\xi$ is a homeomorphisms, an inverse is given by $((\gamma, g), s) \mapsto\left(\gamma,\left(s, g^{-1}\right)\right)$. It is compatible with the left $\Gamma$-actions and the right $G$-actions. We obtain a commutative 
diagram of spaces with left $\Gamma$-actions and right $G$-actions, where the spaces in the lower left and lower right corner carry trivial $G$-actions:

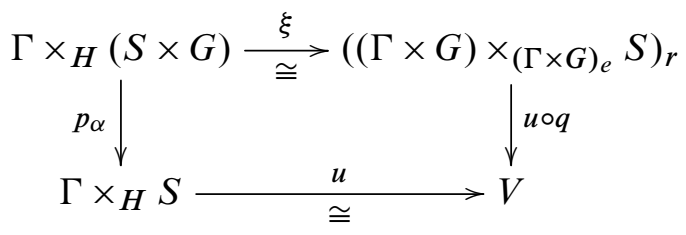

Hence it remains to show that $p_{\alpha}: \Gamma \times_{H}(S \times G) \rightarrow \Gamma / H \times S$ is a principal $G$-bundle after forgetting the $\Gamma$-action.

The Condition (S) ensures that there is an open quasiregular neighborhood $U \subseteq \Gamma / H$ of $1 H$ and a map $s: U \rightarrow \Gamma$ whose composite with the projection pr: $\Gamma \rightarrow \Gamma / H$ is the identity. Define $\bar{s}: \operatorname{pr}^{-1}(U) \rightarrow H$ by $\bar{s}(\gamma)=s \circ \operatorname{pr}(\gamma)^{-1} \cdot \gamma$. It has the property $\bar{s}(\gamma \cdot h)=\bar{s}(\gamma) \cdot h$ for all $\gamma \in p^{-1}(U)$. Define maps

$$
\begin{gathered}
\mu:\left(\operatorname{pr}^{-1}(U) \times_{H} S\right) \times G \rightarrow \operatorname{pr}^{-1}(U) \times_{H}(S \times G), \\
\nu: \operatorname{pr}^{-1}(U) \times_{H}(S \times G) \rightarrow\left(\operatorname{pr}^{-1}(U) \times_{H} S\right) \times G
\end{gathered}
$$

by

$$
\begin{aligned}
& \mu((\gamma, s), g):=\left(\gamma,\left(s, \alpha(\gamma)^{-1} \cdot g\right)\right), \\
& \nu(\gamma,(s, g)):=((\gamma, s), \alpha(\gamma) \cdot g) .
\end{aligned}
$$

Then $\mu$ and $v$ are to one another inverse $G$-homeomorphism. They induce a trivialization of $p_{\alpha}$ restricted to $\operatorname{pr}^{-1}(U) \times_{H} S$ to the trivial principal $G$-bundle over $\operatorname{pr}^{-1}(U) \times_{H} S$.

Since $\gamma H \in \Gamma / H$ is contained in the open subset $\gamma \cdot U$ and $p_{\sigma}$ is a $\Gamma$-equivariant map, we conclude that $p_{\alpha}$ is locally trivial and hence a principal $G$-bundle.

This finishes the proof that $p: E_{r} \rightarrow E / G$ is a principal $G$-bundle. Since $p$ is obviously $\Gamma$-equivariant, $p$ is a $\Gamma$-equivariant principal $G$-bundle. It remains to prove $\mathcal{R}^{\prime}(p) \subseteq \mathcal{R}$ since then $\mathcal{R}(p) \subseteq \mathcal{R}$ holds.

Consider $e \in E$. Its isotropy subgroup $(\Gamma \times G)_{e}$ belongs to $\mathcal{F}(\mathcal{R})$ by assumption. Hence there exists $(H, \alpha) \in \mathcal{R}$ with $(\Gamma \times G)_{e}=K(H, \alpha):=\{(h, \alpha(h) \mid h \in H\}$. This implies that $\Gamma_{p(e)}=H$. We conclude that $\rho_{e}: H \rightarrow G$ is given by $\alpha$ since for $h \in H$ we have $e=(h, \alpha(h))^{-1} \cdot e=\left(h^{-1}, 1\right) \cdot\left(1, \alpha\left(h^{-1}\right)\right) \cdot e$ in the $\Gamma \times G$-space $E$ and hence we get $h \cdot e=e \cdot \alpha(h)$ in $E_{r}$. This finishes the proof of Theorem 8.1. 


\section{Local structure}

Next we deal with the structure of a $\Gamma$-equivariant principal $G$-bundles on small open $\Gamma_{b}$-invariant neighborhoods of points $b$ in the base space.

Theorem 9.1 (Local structure) Let $\mathcal{R}$ be a family of local representations for $(\Gamma, G)$ satisfying Condition (H) introduced in Definition 6.1. Let $p: E \rightarrow B$ be a $\Gamma$-equivariant principal $G$-bundle with $\mathcal{R}(p) \subseteq \mathcal{R}$. Consider any point $b \in B$ and any $\Gamma_{b}$-invariant open neighborhood $W$ of $b \in B$.

Then for every $b$ in $B$ the exists a commutative diagram

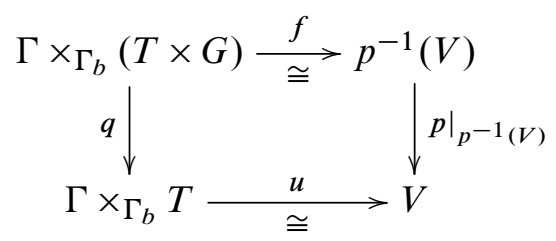

with the following properties.

(i) The subset $T \subseteq B$ contains $b$, satisfies $T \subseteq W$, is $\Gamma_{b}$-invariant and $\Gamma_{b}-$ contractible.

(ii) The subset $V \subseteq B$ is an open $\Gamma$-invariant neighborhood of $b$.

(iii) The group $\Gamma_{b}$ acts from the right on $T \times G$ by

$$
\gamma \cdot(t, g):=\left(\gamma \cdot t, \rho_{e}(\gamma) \cdot g\right)
$$

where $\left(\Gamma_{e}, \rho_{e}\right)$ is the local representation of $p$ associated to a fixed element $e \in E$ with $p(e)=b$; see (3-1).

(iv) The upper vertical map is a homeomorphism compatible with the left $\Gamma$-actions and the right $G$-actions, which at the source is given by

$$
\gamma^{\prime} \cdot((\gamma, t), g) \cdot g^{\prime}=\left(\left(\gamma^{\prime} \gamma, t\right), g g^{\prime}\right)
$$

(v) The lower horizontal arrow is a homeomorphism compatible with the left $\Gamma$ actions, and $q$ sends $((\gamma, t), g)$ to $(\gamma, t)$.

Proof Because of Theorem 8.1(i) we can interpret $E_{l}$ as a $\Gamma \times G-\mathrm{CW}-$ complex. Then the claim follows from the Slice Theorem 7.1 and the proof of Theorem 8.1(ii). More precisely, the desired diagram is the diagram of left $\Gamma \times G$-spaces coming from combining the diagrams (8-2) and (8-3), if we replace $S$ by its image $T$ under $p: E \rightarrow B$. This is possible since the projection $\Gamma \times G \rightarrow \Gamma$ induces an isomorphism $\psi_{e}:(\Gamma \times G)_{e} \stackrel{\cong}{\rightrightarrows} \Gamma_{b}$ and $\left.p\right|_{S}: S \rightarrow T$ is a $\psi_{e}$-homeomorphism. 


\section{Homotopy invariance}

Next we show that the pullback of a $\Gamma$-equivariant principal $G$-bundle with $\Gamma-$ homotopic maps yields isomorphic $\Gamma$-equivariant principal $G$-bundles.

Theorem 10.1 (Homotopy invariance) Let $\mathcal{R}$ be a family of local representations for $(\Gamma, G)$ satisfying Condition $(H)$ introduced in Definition 6.1. Let $B$ be a $\Gamma-C W-$ complex and let $p: E \rightarrow B \times[0,1]$ be a $\Gamma$-equivariant principal $G$-bundle with $\mathcal{R}(p) \subseteq \mathcal{R}$. Let $i_{0}: B=B \times\{0\} \rightarrow B \times[0,1]$ be the inclusion.

Then $i_{0}^{*} E \times[0,1] \stackrel{i_{0}^{*} p \times \operatorname{id}_{[0,1]}}{\longrightarrow} B \times[0,1]$ is a $\Gamma$-equivariant principal $G$-bundle and there exists an isomorphism of $\Gamma$-equivariant principal $G$-bundles

$$
f: i_{0}^{*} E \times[0,1] \rightarrow E
$$

over $B \times[0,1]$ whose restriction to $B \times\{0\}$ is the identity.

Proof Let $p_{n}: E_{n} \rightarrow B_{n}$ be the restriction of $i_{0}^{*} E$ to the $n$-skeleton $B_{n}$ of $B$. We will construct inductively over $n$ an isomorphism of $\Gamma$-equivariant principal $G$-bundles

$$
f_{n}: E_{n} \times\left.[0,1] \stackrel{\cong}{\rightarrow} E\right|_{B_{n} \times[0,1]}
$$

such that the restriction of $f_{n}$ to $B_{n} \times\{0\}$ is the identity and the restriction of $f_{n}$ to $B_{n-1} \times[0,1]$ is $f_{n-1}$. Then we can define the desired isomorphism $f$ by requiring that $\left.f\right|_{B_{n} \times[0,1]}=f_{n}$, since $i_{0}^{*} E$ has the weak topology with respect to the filtration by the $E_{n}-$ s by Lemma A.6(i) and (ii).

The induction beginning $n=-1$ is trivial, the induction step from $(n-1)$ to $n$ done as follows. Choose a $\Gamma$-pushout

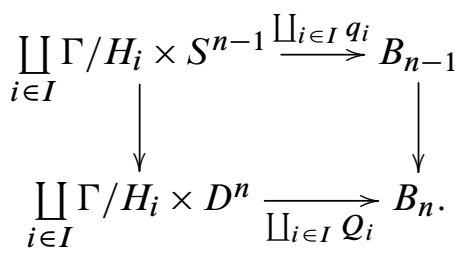

From Lemma A.5(i) and (ii) we obtain a $\Gamma \times G$-pushout

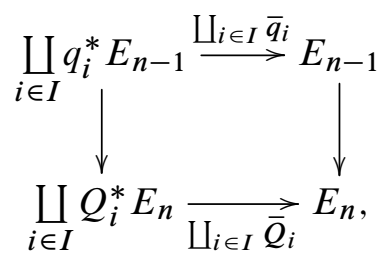


and thus a $\Gamma \times G$-pushout

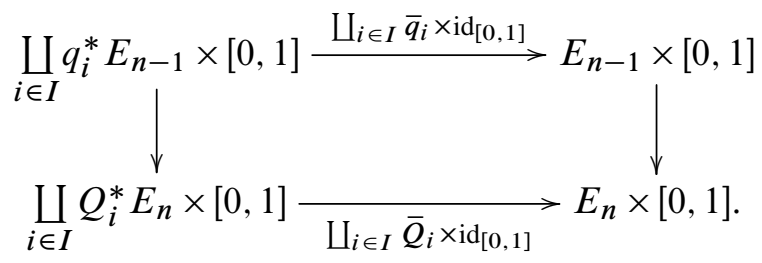

Hence it suffices to extend for each $i \in I$ the map of $\Gamma$-equivariant principal $G$-bundles over $\Gamma / H_{i} \times S^{n-1} \times[0,1]$,

$$
x_{i}: q_{i}^{*} E_{n-1} \times[0,1] \stackrel{\bar{q}_{i} \times[0,1]}{\longrightarrow} E_{n-1} \times\left.[0,1] \stackrel{f_{n-1}}{\longrightarrow} E\right|_{B_{n-1} \times[0,1]},
$$

covering $q_{i} \times \mathrm{id}_{[0,1]}: \Gamma / H_{i} \times S^{n-1} \times[0,1] \rightarrow B_{n-1} \times[0,1]$ to a map of $\Gamma$-equivariant principal $G$-bundles over $\Gamma / H_{i} \times D^{n} \times[0,1]$,

$$
y_{i}: Q_{i}^{*} E_{n} \times\left.[0,1] \rightarrow E\right|_{B_{n} \times[0,1]},
$$

covering $Q_{i} \times \operatorname{id}_{[0,1]}: \Gamma / H_{i} \times D^{n} \times[0,1] \rightarrow B_{n} \times[0,1]$ such that the restriction of $y_{i}$ to $\Gamma / H_{i} \times D^{n} \times\{0\}$ is fiberwise the identity. We obtain from $x_{i}$ a map of $\Gamma$-equivariant principal $G$-bundles over $\Gamma / H_{i} \times S^{n-1} \times[0,1]$,

$$
x_{i}^{\prime}: q_{i}^{*} E_{n-1} \times\left.[0,1] \rightarrow\left(Q_{i} \times \operatorname{id}_{[0,1]}\right)^{*} E\right|_{\Gamma / H_{i} \times S^{n-1} \times[0,1]},
$$

covering the identity id: $\Gamma / H_{i} \times S^{n-1} \times[0,1] \rightarrow \Gamma / H_{i} \times S^{n-1} \times[0,1]$ such that the restriction of $x_{i}^{\prime}$ to $\Gamma / H_{i} \times S^{n-1} \times\{0\}$ is the identity. It remains to extend $x_{i}^{\prime}$ to map of $\Gamma$-equivariant principal $G$-bundles over $\Gamma / H_{i} \times D^{n} \times[0,1]$,

$$
y_{i}^{\prime}: Q_{i}^{*} E_{n} \times[0,1] \rightarrow\left(Q_{i} \times \operatorname{id}_{[0,1]}\right)^{*} E,
$$

covering the identity id: $\Gamma / H_{i} \times D^{n} \times[0,1] \rightarrow \Gamma / H_{i} \times D^{n} \times[0,1]$ such that the restriction of $y_{i}^{\prime}$ to $\Gamma / H_{i} \times D^{n} \times\{0\}$ is the identity. We obtain from (8-1), now applied to $D^{n} \times[0,1]$ instead of $D^{n}$, isomorphisms of $\Gamma$-equivariant principal $G$-bundles over $\Gamma / H_{i} \times D^{n} \times[0,1]$,

$$
\begin{aligned}
& a:(\Gamma \times G) / H_{i} \times D^{n} \times[0,1] \stackrel{\cong}{\rightrightarrows} Q_{i}^{*} E_{n} \times[0,1], \\
& b:(\Gamma \times G) / H_{i} \times D^{n} \times[0,1] \stackrel{\cong}{\rightrightarrows}\left(Q_{i} \times \operatorname{id}_{[0,1]}\right)^{*} E,
\end{aligned}
$$

for an appropriate subgroup $H_{i} \subseteq \Gamma \times G$ if we convert the left $G$-action into a right $G$-action in the usual way. By conjugation with the restrictions of $a$ and $b^{-1}$ to $\Gamma / H_{i} \times S^{n-1} \times[0,1]$, we obtain from $x_{i}^{\prime}$ an isomorphism of $\Gamma$-equivariant principal 
$G$-bundles over $\Gamma / H_{i} \times S^{n-1} \times[0,1]$,

$$
x_{i}^{\prime \prime}:(\Gamma \times G) / H_{i} \times S^{n-1} \times[0,1] \stackrel{\cong}{\rightarrow}(\Gamma \times G) / H_{i} \times S^{n-1} \times[0,1] .
$$

The remaining problem is to extend $x_{i}^{\prime \prime}$ to an isomorphism of $\Gamma$-equivariant principal $G$-bundles over $\Gamma / H_{i} \times D^{n} \times[0,1]$,

$$
y_{i}^{\prime \prime}:(\Gamma \times G) / H_{i} \times D^{n} \times[0,1] \stackrel{\cong}{\longrightarrow}(\Gamma \times G) / H_{i} \times D^{n} \times[0,1],
$$

whose restriction to $D^{n} \times\{0\}$ is the restriction of $b^{-1} \circ a$ to $D^{n} \times\{0\}$. Notice that $x_{i}^{\prime \prime}$ is the same as a map $S^{n-1} \times[0,1] \rightarrow \operatorname{map}_{\Gamma \times G}\left((\Gamma \times G) / H_{i},(\Gamma \times G) / H_{i}\right)$ and $y_{i}^{\prime \prime}$ is the same as map $D^{n} \times[0,1] \rightarrow \operatorname{map}_{\Gamma \times G}\left((\Gamma \times G) / H_{i},(\Gamma \times G) / H_{i}\right)$. Hence the remaining problem is to extend a given map

$$
S^{n-1} \times[0,1] \cup D^{n} \times\{0\} \rightarrow \operatorname{map}_{\Gamma \times G}\left((\Gamma \times G) / H_{i},(\Gamma \times G) / H_{i}\right)
$$

to a map

$$
D^{n} \times[0,1] \rightarrow \operatorname{map}_{\Gamma \times G}\left((\Gamma \times G) / H_{i},(\Gamma \times G) / H_{i}\right) .
$$

This is possible since there is a retraction $D^{n} \times[0,1] \rightarrow S^{n-1} \times[0,1] \cup D^{n} \times\{0\}$.

\section{Universal equivariant principal bundles}

Fix a family of local representations $\mathcal{R}$ which satisfy Condition $(\mathrm{H})$ introduced in Definition 6.1. In this section we construct the universal $\Gamma$-equivariant principal $G-$ bundle and in particular the classifying space for $\Gamma$-equivariant principal $G$-bundles with respect to $\mathcal{R}$.

Definition 11.1 (Compatibility) We call $\mathcal{R}$ compatible with the $\Gamma$-CW-complex $X$ if for any $x \in X$ and $(H, \alpha) \in \mathcal{R}$ with $\Gamma_{x} \subseteq H$ the pair $\left(\Gamma_{x},\left.\alpha\right|_{\Gamma_{x}}\right)$ belongs to $\mathcal{R}$ again.

Remark 11.2 Notice that this condition is automatically satisfied for every $\Gamma-C W-$ complex if $\mathcal{R}$ is closed under taking subgroups, ie for $(H, \alpha)$ in $\mathcal{R}$ and $K \subseteq H$ we have $\left(K,\left.\alpha\right|_{K}\right) \in \mathcal{R}$.

Consider $\Gamma-\mathrm{CW}$-complexes $X$ and $B$ and a $\Gamma$-equivariant principal $G$-bundle $p: E \rightarrow B$ over the $\Gamma-\mathrm{CW}$-complex $B$ with $\mathcal{R}(p) \subseteq \mathcal{R}$. Let $\operatorname{Bundle}_{\Gamma, G, \mathcal{R}}(X)$ be the set of isomorphism classes of $\Gamma$-equivariant principal $G$-bundles $q: E \rightarrow X$ with $\mathcal{R}(q) \subseteq \mathcal{R}$. Suppose that $\mathcal{R}$ is compatible with $X$. Then for any $\Gamma$-map $f: X \rightarrow B$ 
the pullback $f^{*} p$ is a $\Gamma$-equivariant principal $G$-bundle with $\mathcal{R}\left(f^{*} p\right) \subseteq \mathcal{R}$. Because of Theorem 10.1 the pullback construction yields a well-defined map

$$
c:[X, B]^{\Gamma} \rightarrow \operatorname{Bundle}_{\Gamma, G, \mathcal{R}}(X), \quad[f] \mapsto\left[f^{*} p\right] .
$$

Notation 11.3 (Classifying space of a family of subgroups) Given group $G$ and a family $\mathcal{F}$ of subgroups of $G$, denote by $E_{\mathcal{F}}(G)$ the classifying space of the family $\mathcal{F}$.

Recall that $E_{\mathcal{F}}(G)$ is a $G-\mathrm{CW}$-complex whose isotropy groups belong to $\mathcal{F}$ and for which the $H$-fixed point set $E_{\mathcal{F}}(G)^{H}$ is nonempty and weakly contractible for every $H \in \mathcal{F}$. A model always exists. For any $G-\mathrm{CW}$-complex $X$ whose isotropy groups belong to $\mathcal{F}$, there is up to $G$-homotopy precisely one $G$-map $X \rightarrow E_{\mathcal{F}}(G)$. In particular two models for $E_{\mathcal{F}}(G)$ are $G$-homotopy equivalent. For more information about classifying spaces of a family we refer for instance to the first author [23].

Theorem 11.4 (Classifying space for $\Gamma$-equivariant principal $G$-bundles with family of local representatives contained in $\mathcal{R}$ satisfying Condition $(\mathrm{H})$ ) Let $\mathcal{R}$ be a family of local representations for $(\Gamma, G)$ satisfying Condition $(H)$ introduced in Definition 6.1. Define

$$
\begin{aligned}
E(\Gamma, G, \mathcal{R}) & :=E_{\mathcal{F}(\mathcal{R})}(\Gamma \times G)_{r}, \\
B(\Gamma, G, \mathcal{R}) & :=E_{\mathcal{F}(\mathcal{R})}(\Gamma \times G)_{r} / G,
\end{aligned}
$$

where $\mathcal{F}(\mathcal{R})$ is the family of subgroups of $\Gamma \times G$ introduced in Lemma 3.5. Let $p: E(\Gamma, G, \mathcal{R}) \rightarrow B(\Gamma, G, \mathcal{R})$ be the projection. Let $X$ be a $\Gamma-C W-$ complex such that $\mathcal{R}$ is compatible with $X$ in the sense of Definition 11.1.

Then $p: E(\Gamma, G, \mathcal{R}) \rightarrow B(\Gamma, G, \mathcal{R})$ is a $\Gamma$-equivariant principal $G$-bundle and the map $c$ defined in (11-1),

$$
c:[X, B]^{\Gamma} \rightarrow \operatorname{Bundle}_{\Gamma, G, \mathcal{R}}(X), \quad[f] \mapsto\left[f^{*} p\right],
$$

is bijective.

Proof We conclude from Theorem 8.1 (ii) that $p: E(\Gamma, G, \mathcal{R}) \rightarrow B(\Gamma, G, \mathcal{R})$ is a $\Gamma$-equivariant principal $G$-bundle.

We construct an inverse map

$$
d: \operatorname{Bundle}_{\Gamma, G, \mathcal{R}}(X) \rightarrow[X, B]^{\Gamma}
$$

as follows.

Let $q: E \rightarrow B$ be a $\Gamma$-equivariant principal $G$-bundle whose family of local representations is contained in $\mathcal{R}$. We conclude from Theorem 8.1(i) that $E_{l}$ is a left 
$\Gamma \times G-\mathrm{CW}$-complex whose isotropy groups belong to $\mathcal{F}(\mathcal{R})$. Hence there is up to $\Gamma \times G$-homotopy precisely one $\Gamma \times G$-map $\bar{f}: E_{l} \rightarrow E_{\mathcal{F}(\mathcal{R})}(\Gamma \times G)$ which is the same a map $\bar{f}: E \rightarrow E(\Gamma, G, \mathcal{R})$ compatible with the left $\Gamma$-actions and right $G$-actions. Taking the $G$-quotient yields a map $f: B \rightarrow B(\Gamma, G, \mathcal{R})$ which is unique up to $\Gamma$-homotopy and for which the following diagram commutes:

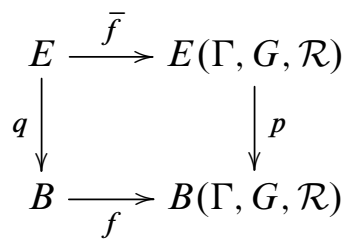

Hence $q$ is up to isomorphism the pullback of the universal bundle $p$ with the $\Gamma-$ map $f: B \rightarrow B(\Gamma, G, \mathcal{R})$ and the construction of this $\Gamma$-map $f$ is unique up to $\Gamma$-homotopy. Define $d([q])$ by $[f]$. This is well defined since obviously $[f]$ depends only on the isomorphism class $[q]$ of $q$. Since $f^{*} p$ is isomorphic to $q$, we get $c \circ d=\operatorname{id}_{B_{\text {undle }}, G, \mathcal{R}}(X)$. One easily checks $d \circ c=\operatorname{id}_{[X, B] \Gamma}$.

This finishes the proof of Theorem 11.4.

We will call $p: E(\Gamma, G, \mathcal{R}) \rightarrow B(\Gamma, G, \mathcal{R})$ appearing in Theorem 11.4 the universal $\Gamma$ equivariant principal $G$-bundle with respect to the family of local representations $\mathcal{R}$. If $p^{\prime}: E(\gamma, G, \mathcal{R})^{\prime} \rightarrow B(\gamma, G, \mathcal{R})^{\prime}$ is another such universal bundle, then there is a commutative diagram of such bundles covering a $\Gamma$-homotopy equivalence $f$ :

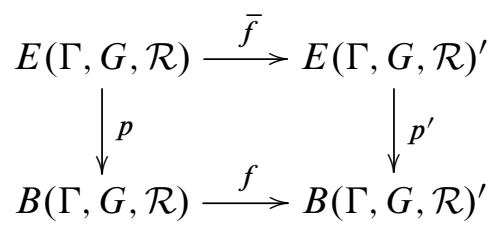

That is, $\bar{f}$ is compatible with the left $\Gamma$ and the right $G$-actions and is a homeomorphism. Such a diagram is unique up to $\Gamma \times G$-homotopy, in particular the $\Gamma$-map $f$ is unique up to $\Gamma$-homotopy.

\section{Reduction of the structure group to a maximal compact subgroup}

If $K$ is a subgroup of $G$ and $p_{0}: E_{0} \rightarrow B$ is a $\Gamma$-equivariant principal $K$-bundle, then

$$
\bar{p}_{0}: E_{0} \times_{K} G \rightarrow B, \quad(e, g) \mapsto p_{0}(e)
$$


inherits the structure of a $\Gamma$-equivariant principal $G$-bundle in the obvious way. One has just to verify local triviality of the underlying principal $G$-bundle $\bar{p}_{0}$.

Definition 12.1 (Reduction) Let $p: E \rightarrow B$ be a $\Gamma$-equivariant principal $G$-bundle. Given a subgroup $K \subseteq G$, a reduction to a $\Gamma$-equivariant principal $K$-bundle is a $\Gamma$-equivariant principal $K$-bundle $p_{0}: E_{0} \rightarrow B$ together with an isomorphism of $\Gamma$-equivariant principal $G$-bundles from $\bar{p}_{0}$ to $p$.

Lemma 12.2 Let $p: E \rightarrow B$ be a $\Gamma$-equivariant principal $G$-bundle and $K \subseteq G$ be a subgroup. Let $f: E \rightarrow G / K$ be a $\Gamma \times G$-map, where we equip the target with the $\Gamma \times G$-action given by $(\gamma, g) \cdot g^{\prime} K=g g^{\prime} K$. Suppose that the pair $(G, K)$ satisfies Condition (S); see Definition 4.1. Put $E_{f}=f^{-1}(1 K)$. Let $p_{f}: E_{f} \rightarrow B$ be the restriction of $p$ to $E_{f}$. Then:

(i) The map $p_{f}: E_{f} \rightarrow B$ is a $\Gamma$-equivariant principal $K$-bundle which is a $K$ reduction of $p$.

(ii) Every $K$-reduction of $p: E \rightarrow B$ is up to isomorphism of $\Gamma$-equivariant principal $K$-bundles of the form $p_{f}: E_{f} \rightarrow B$ for appropriate $f$.

(iii) If $f_{0}, f_{1}: E \rightarrow G / K$ are $\Gamma \times G$-maps which are $\Gamma \times G$-homotopic, then $p_{f_{0}}: E_{f_{0}} \rightarrow B$ and $p_{f_{1}}: E_{f_{1}} \rightarrow B$ are isomorphic as $\Gamma$-equivariant principal K-bundles.

Proof (i) From Lemma 4.4 we obtain a $\Gamma \times G$-homeomorphism

$$
u: E_{0} \times_{K} G \stackrel{\cong}{\longrightarrow} E, \quad(g, e) \mapsto e \cdot g .
$$

It remains to show that $p_{0}: E_{0} \rightarrow B$ is a principal $K$-bundle, ie to show local triviality. Since $p$ is locally trivial, it suffices to treat the case, where $E=G \times B$ and $p: G \times B \rightarrow$ $B$ is the projection. Choose a quasiregular open subset $U \subseteq G / K$ with $1 K \in U$ and a map $s: U \rightarrow G$ satisfying $\operatorname{pr} \circ S=\operatorname{id}_{U}$, where pr: $G \rightarrow G / K$ is the projection. Let $V$ be the open subset of $B$ given by $p\left(f^{-1}(U)\right)$. Since $V$ is $\Gamma$-invariant, it is quasiregular by Lemma A.1(vi). Let $\alpha: V \rightarrow G$ be the map sending $v$ to $s \circ f(v, 1)$. Then we obtain an automorphism of the trivial principal $G$-bundle $G \times B \rightarrow G \times B$,

$$
\bar{\alpha}: G \times V \stackrel{\cong}{\longrightarrow} G \times V, \quad(g, v) \mapsto(g \cdot \alpha(v), v) .
$$

If $\pi: G \times V \rightarrow G / K$ sends $(g, v)$ to $g K$, then $\pi \circ \bar{\alpha}=f$. Hence $\bar{\alpha}$ induces a commutative diagram of $K$-spaces

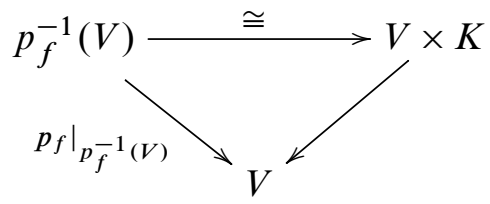


such that the horizontal arrow is a $K$-homeomorphism and the right vertical arrow is the projection.

(ii) Let $p_{0}: E_{0} \rightarrow B$ be a $K$-reduction of $p$. Choose an isomorphism of $\Gamma$-equivariant principal $G$-bundles $u: G \times{ }_{K} E_{0} \rightarrow E$. If we take $f: E \rightarrow G / K$ to be the composite of $u^{-1}$ with the obvious projection $G \times_{K} E_{0} \rightarrow G / K$, then $p_{0}$ is $p_{f}$.

(iii) Fix a $\Gamma \times G$-homotopy $h: E \times[0,1] \rightarrow G / K$ with $h_{k}=f_{k}$ for $k=0,1$. Let $q: E \times[0,1] \rightarrow B \times[0,1]$ be the $\Gamma$-equivariant principal $G$-bundle given by $p \times \operatorname{id}_{[0,1]}$. Then we obtain a $\Gamma$-equivariant principal $K$-bundle $q_{h}:(E \times[0,1])_{h} \rightarrow B \times[0,1]$ whose restriction to $B \times\{k\}$ is $p_{f_{k}}$ for $k=0,1$. Now the claim follows from Theorem 10.1.

Definition 12.3 (Almost connected group) Given a group $G$, let $G^{0}$ be its component of the identity and define the component group $\bar{G}$ by $\bar{G}=G / G^{0}$. We call $G$ almost connected if its component group $\bar{G}$ is compact.

The next result is due to Abels [1, Corollary 4.14].

Theorem 12.4 (Almost connected groups) Let $G$ be a locally compact Hausdorff topological group. Suppose that $G$ is almost connected.

Then $G$ contains a maximal compact subgroup $K$ which is unique up to conjugation, and the $H$-fixed point set $(G / K)^{H}$ is contractible for every compact subgroup $H \subseteq K$.

Theorem 12.5 (Existence of a $K$-reduction) Let $G$ and $\Gamma$ be locally compact second countable topological groups. Suppose that $G$ is almost connected. Let $K \subseteq G$ be a maximal compact subgroup. Let $\mathcal{R}$ be a family of local representations for $(\Gamma, G)$ such that for each element $(H, \alpha)$ in $\mathcal{R}$ the subgroup $H \subseteq \Gamma$ is compact. Let $\mathcal{R}_{K}$ be the family of local representations of $(\Gamma, K)$ which is given by $\mathcal{R}_{K}=\{(H, \alpha) \mid \alpha(H) \subseteq$ $K,(H, \alpha) \in \mathcal{R}\}$.

Then every $\Gamma$-equivariant principal $G$-bundle $p: E \rightarrow B$ with $\mathcal{R}(p) \subseteq \mathcal{R}$ has a (preferred) $K$-reduction $p_{0}: E_{0} \rightarrow B$ which is unique up to isomorphism of $\Gamma$-equivariant principal $K$-bundles and satisfies $\mathcal{R}\left(p_{0}\right) \subseteq \mathcal{R}_{K}$.

Proof Condition $(H)$ is always satisfied under the conditions of Theorem 12.5 because of Theorem 6.3. It suffices to prove the claim for the universal $\Gamma$-equivariant principal $G$-bundle because of Theorem 11.4 , since $K$-reductions are compatible with pullbacks.

Let $p: E(\Gamma, G, \mathcal{R}) \rightarrow B(\Gamma, G, \mathcal{R})$ be the universal $\Gamma$-equivariant principal $G$-bundle with respect to the family $\mathcal{R}$; see Theorem 11.4 . Recall that $E(\Gamma, G, \mathcal{R})$ is a $\Gamma \times G-\mathrm{CW}-$ complex whose isotropy groups belong to the family of subgroups $\mathcal{F}(\mathcal{R})$ associated 
to $\mathcal{R}$. For any element in $K(H, \alpha) \in \mathcal{F}$ the image of $K(H, \alpha)$ under the projection $\Gamma \times G \rightarrow G$ is compact and hence a closed subgroup of $G$. Lemma A.7 implies that $E(\Gamma, G, \mathcal{R}) / \Gamma$ is a $G-\mathrm{CW}$-complex. Because of Theorem 12.4 the $G-\mathrm{CW}$-complex $G / K$ is the classifying space for proper $G$-actions. Hence there is up to $G$-homotopy precisely one map $E(\Gamma, G, \mathcal{R}) / \Gamma \rightarrow G / K$. We conclude that there is up to $\Gamma \times G-$ homotopy precisely one $\Gamma \times G$-map $f: E(\Gamma, G, \mathcal{R}) \rightarrow G / K$. From Lemma 12.2 we obtain a $K$-reduction $p_{f}$ of $p$ which is unique up to isomorphism of $\Gamma$-equivariant principal $K$-bundles. One easily checks $\mathcal{R}\left(p_{f}\right) \subseteq \mathcal{R}_{K}$.

Example 12.6 (Equivariant vector bundles and Riemannian metrics) In the case $G=$ $\mathrm{GL}_{n}(\mathbb{R})$, we have $O(n)$ as the maximal compact subgroup and Theorem 12.5 implies the well-known statement that any equivariant vector bundle $\xi$ over a proper $\Gamma-\mathrm{CW}-$ complex can be equipped with a $\Gamma$-invariant Riemannian metric and that two equivariant vector bundles over a proper $\Gamma-\mathrm{CW}$-complex with $\Gamma$-invariant Riemannian metrics admit an isomorphism respecting the $\Gamma$-invariant Riemannian metrics if and only the equivariant vector bundles are isomorphic (after forgetting the invariant Riemannian metrics).

\section{On the homotopy type of the classifying space}

In this section we want to establish for $H \subseteq \Gamma$ a weak homotopy equivalence

$$
\bigsqcup_{[\alpha] \in \operatorname{hom}_{\mathcal{R}}(H, G) / G} B C_{G}(\alpha) \simeq B(\Gamma, G, \mathcal{R})^{H},
$$

where $[\alpha]$ runs over the $G$-conjugacy classes of homomorphisms $\alpha: H \rightarrow G$ with $(H, \alpha) \in \mathcal{R}$. This will follow from Theorem 13.1.

Let $\operatorname{hom}(H, G)$ be the space of homomorphisms of topological groups $H \rightarrow G$, endowed with the subspace topology from $\operatorname{hom}(H, G) \subseteq \operatorname{map}(H ; G)$; see Section A.5. Denote by $\operatorname{hom}(H, G) / G$ the quotient space under the conjugation action of $G$, ie, the left $G$-action sending $(g, \alpha) \in G \times \operatorname{hom}(H, G)$ to $c_{g} \circ \alpha$, where $c_{g}: G \rightarrow G$ sends $g^{\prime}$ to $g g^{\prime} g^{-1}$; see Section A.5. Recall that the centralizer of $\alpha \in \operatorname{hom}(H, G)$ is

$$
C_{G}(\alpha):=\left\{g \in G \mid g \alpha(h) g^{-1}=\alpha(h) \text { for all } h \in H\right\} .
$$

For $\mathcal{R}$ a family of local representations for $(\Gamma, G)$, and $(H, \alpha)$ in $\mathcal{R}$ define

$$
\operatorname{hom}_{\mathcal{R}}(H, G):=\{\alpha \in \operatorname{hom}(H, G) \mid(H, \alpha) \in \mathcal{R}\}
$$

and note that $\operatorname{hom}_{\mathcal{R}}(H, G)$ is closed under the conjugation of $G$. 
Theorem 13.1 (Fixed point sets of $B(\Gamma, G, \mathcal{R})$ ) Let $\mathcal{R}$ be a family of local representations for $(\Gamma, G)$ satisfying Condition $(H)$ introduced in Definition 6.1. Consider an element $(H, \alpha)$ in $\mathcal{R}$.

(i) We obtain a bijection

$$
\operatorname{hom}_{\mathcal{R}}(H, G) / G \stackrel{\cong}{\rightarrow} \pi_{0}\left(B(\Gamma, G, \mathcal{R})^{H}\right) .
$$

(ii) Given $(H, \alpha)$ in $\mathcal{R}$, let $B(\Gamma, G, \mathcal{R})_{\alpha}^{H}$ be the path component of $B(\Gamma, G, \mathcal{R})^{H}$ that corresponds under the bijection of (i) to the class of $\alpha$ in $\operatorname{hom}_{\mathcal{R}}(H, G) / G$. Then there exists a weak homotopy equivalence

$$
B C_{G}(\alpha) \stackrel{\simeq}{\rightarrow} B(\Gamma, G, \mathcal{R})_{\alpha}^{H} .
$$

Proof (i) We obtain from Theorem 11.4 bijections

$$
\pi_{0}\left(B(\Gamma, G, \mathcal{R})^{H}\right) \stackrel{\cong}{\rightarrow}[\Gamma / H, B(\Gamma, G, \mathcal{R})]^{\Gamma} \stackrel{\cong}{\rightarrow} \operatorname{Bundle}_{\Gamma, G, \mathcal{R}}(\Gamma / H) .
$$

Given $\alpha \in \operatorname{hom}_{\mathcal{R}}(H, G)$, we obtain a $\Gamma$-equivariant principal $G$-bundle

$$
\xi_{\alpha}: \Gamma \times_{\alpha} G \rightarrow \Gamma / H, \quad(\gamma, g) \mapsto \gamma H,
$$

where $\Gamma \times{ }_{\alpha} G$ is the quotient of $\Gamma \times G$ under the left $H$-action given by $h \cdot(\gamma, g)=$ $\left(\gamma \cdot h^{-1}, \alpha(h) \cdot g\right)$. We conclude from Lemma 5.1 (ii) and (iii) that any $\Gamma$-equivariant principal $G$-bundle $q: E \rightarrow \Gamma / H$ with $\mathcal{R}(q) \subset \mathcal{R}$ is isomorphic to $\xi_{\alpha}$ for some $\alpha \in \operatorname{hom}_{\mathcal{R}}(H, G)$, and for two elements $\alpha, \beta \in \operatorname{hom}_{\mathcal{R}}(H, G)$ the $\Gamma$-equivariant principal $G$-bundles $\xi_{\alpha}$ and $\xi_{\beta}$ are isomorphic if and only if the classes of $\alpha$ and $\beta$ in $\operatorname{hom}_{\mathcal{R}}(H, G) / G$ agree. Hence we obtain a bijection

$$
\operatorname{hom}_{\mathcal{R}}(H, G) / G \stackrel{\cong}{\rightrightarrows} \operatorname{Bundle}_{\Gamma, G, \mathcal{R}}(\Gamma / H), \quad[\alpha] \mapsto\left[\xi_{\alpha}\right] .
$$

(ii) Let $p: E(\Gamma, G, \mathcal{R}) \rightarrow B(\Gamma, G, \mathcal{R})$ be the canonical projection which is a principal $G$-bundle. We want to show that $p$ induces a principal $C_{G}(\alpha)$-bundle

$$
p_{(H, \alpha)}: E(\Gamma, G, \mathcal{R})^{K(H, \alpha)} \rightarrow B(\Gamma, G, \mathcal{R})_{\alpha}^{H} .
$$

Abbreviate $E=E(\Gamma, G, \mathcal{R})$. Consider $(H, \alpha) \in \mathcal{R}$. Let $E^{\langle H\rangle}$ be the subspace of $E$ consisting of those elements $e \in E$ such that for each $h \in H$ there exists $g \in G$ with $h \cdot e=e \cdot g$. For each $e \in E$ define $\rho_{e} \in \operatorname{hom}(H, G)$ by requiring $h \cdot e=e \cdot \rho_{e}(h)$. Thus we can define a map of sets

$$
\rho: E^{\langle H\rangle} \rightarrow \operatorname{hom}(H, G) .
$$


Next we show that $\rho$ is continuous. We apply Theorem 9.1 at the point $b=p(e)$ and obtain a commutative diagram

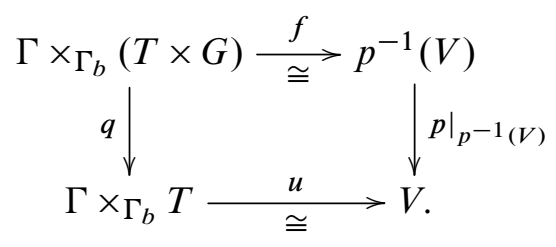

It suffices to show the composite of $\rho$ with the map $f^{\langle H\rangle}:\left(\Gamma \times \Gamma_{b}(T \times G)\right)^{\langle H\rangle} \rightarrow E^{\langle H\rangle}$ is continuous.

Put $\Gamma^{\langle H\rangle}=\left\{\gamma \in \Gamma \mid \gamma^{-1} \cdot H \cdot \gamma \subseteq \Gamma_{b}\right\}$. Consider the commutative diagram

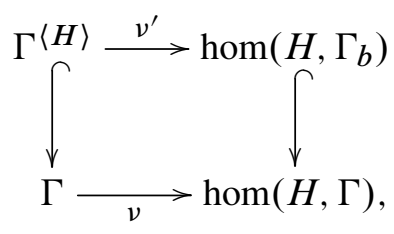

where $v^{\prime}$ and $v$ respectively are given by conjugating the inclusion homomorphism $i: H \rightarrow \Gamma_{b}$ with $\gamma \in \Gamma^{\langle H\rangle}$ and $\gamma \in \Gamma$ respectively, the left vertical arrow is the inclusion of subgroups and the right vertical arrow is the injection induced by the inclusion $\Gamma_{b} \rightarrow \Gamma$. Since $\operatorname{map}\left(H, \Gamma_{b}\right)$ is the preimage of the constant map under $\operatorname{map}(H, \Gamma) \rightarrow \operatorname{map}\left(H, \Gamma / \Gamma_{b}\right)$, we conclude from Lemma A.2 that the right vertical arrow is the inclusion of a closed subspace. As explained in Lemma A.2, the conjugation map $\Gamma \times \operatorname{hom}(H, \Gamma) \rightarrow \operatorname{hom}(H, \Gamma)$ is continuous. We conclude that $v$ and hence $v^{\prime}$ are continuous. Define a map of sets

$$
\mu: \Gamma^{\langle H\rangle} \times G \rightarrow \operatorname{hom}(H, G)
$$

by sending $(\gamma, g)$ to the composite $H \stackrel{c_{\gamma} \circ i}{\longrightarrow} \Gamma_{b} \stackrel{\rho_{e}}{\longrightarrow} G \stackrel{c_{g}}{\longrightarrow} G$, where $i: H \rightarrow \Gamma_{b}$ is the inclusion. Since $v^{\prime}$ is continuous, $\mu$ is continuous. The map $\mu$ factorizes through the obvious projection $\Gamma^{\langle H\rangle} \times G \rightarrow\left(\Gamma \times \Gamma_{b} G\right)^{\langle H\rangle}$, which is an identification, to a continuous map $\bar{\mu}:\left(\Gamma \times_{\Gamma_{b}} G\right)^{\langle H\rangle} \rightarrow \operatorname{hom}(H, G)$ making the following diagram commutative:

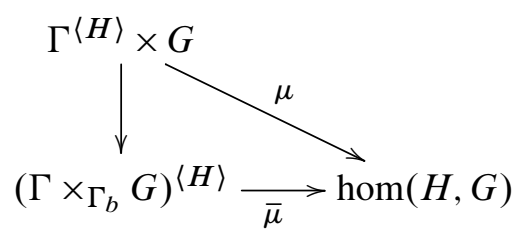


If pr: $\Gamma \times \Gamma_{b}(T \times G) \rightarrow \Gamma \times \Gamma_{b} G$ is the projection, the following diagram is commutative:

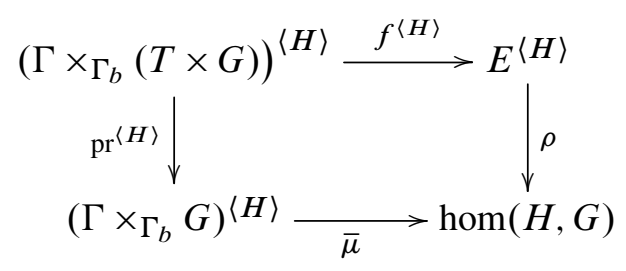

Hence $\bar{\mu} \circ \operatorname{pr}^{\langle H\rangle}=\rho \circ f^{\langle H\rangle}$. This implies $\rho \circ f^{\langle H\rangle}$ and hence $\rho: E^{\langle H\rangle} \rightarrow \operatorname{hom}(H, G)$ is continuous. One easily checks that $\rho$ is a $G$-map.

Let $E^{\langle H, \alpha\rangle}$ be the preimage under $\rho$ of the orbit $G \cdot \alpha \subseteq \operatorname{hom}(H, G)$ with respect to the action of $G$ on $\operatorname{hom}(H, G)$ given by composing with conjugation automorphisms. Hence $\rho$ induces a $G$-map

$$
\rho_{\alpha}^{\prime}: E^{\langle H, \alpha\rangle} \rightarrow G \cdot \alpha .
$$

Since $\mathcal{R}$ satisfies Condition (H), the $G$-map $\iota_{\alpha}: G / C_{G}(\alpha) \rightarrow G \cdot \alpha, g \mapsto c_{g} \circ \alpha$ is a homeomorphism. Define the $G$-map

$$
\rho_{\alpha}=\iota_{\alpha}^{-1} \circ \rho_{\alpha}^{\prime}: E^{\langle H, \alpha\rangle} \rightarrow G / C_{G}(\alpha) .
$$

One easily checks that the preimage of $1 \cdot C_{G}(\alpha) \in G / C_{G}(\alpha)$ under $\rho_{\alpha}$ is $E^{K(H, \alpha)}$. Since $\left(G, C_{G}(\alpha)\right)$ satisfies Condition (S), it implies that the canonical $G$-map

$$
G \times C_{G}(\alpha) E^{K(H, \alpha)} \rightarrow E^{\langle H, \alpha\rangle}
$$

is a $G$-homeomorphism. One easily checks $E^{\langle H, \alpha\rangle}$ is the preimage of $B(\Gamma, G, \mathcal{R})_{\alpha}^{H}$ under $p: E(\Gamma, G, \mathcal{R}) \rightarrow B(\Gamma, G, \mathcal{R})$. Hence $p$ induces a $C_{G}(\alpha)$-map

$$
p_{(H, \alpha)}: E(\Gamma, G, \mathcal{R})^{K(H, \alpha)} \rightarrow B(\Gamma, G, \mathcal{R})_{\alpha}^{H}
$$

for which the following diagram of $G$-spaces commutes:

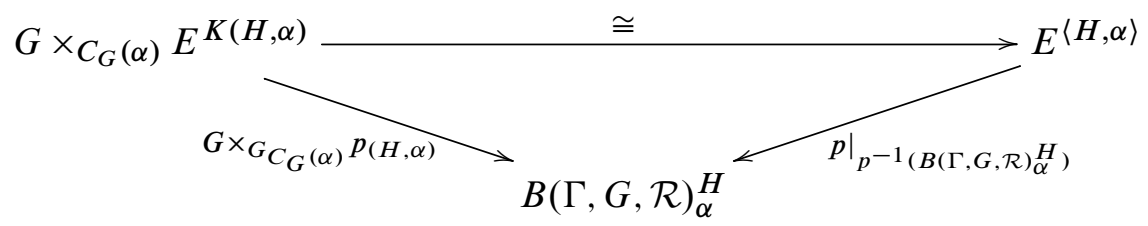

Since the right vertical arrow is a principal $G$-bundle, Lemma 12.2 implies that $p_{(H, \alpha)}: E(\Gamma, G, \mathcal{R})^{K(H, \alpha)} \rightarrow B(\Gamma, G, \mathcal{R})_{\alpha}^{H}$ is a principal $C_{G}(\alpha)$-bundle.

Let $X$ be a CW-complex and $f: X \rightarrow B(\Gamma, G, \mathcal{R})_{\alpha}^{H}$ be a weak homotopy equivalence. Let $q: f^{*} E(\Gamma, G, \mathcal{R})^{K(H, \alpha)} \rightarrow X$ be the pullback of the principal $C_{G}(\alpha)$-bundle $p_{(H, \alpha)}$. Every principal $G$-bundle is a fibration. We conclude from the long exact 
sequence of homotopy groups associated to $p_{(H, \alpha)}$ and $q$ and the five lemma that the induced map $f^{*} E(\Gamma, G, \mathcal{R})^{K(H, \alpha)} \rightarrow E(\Gamma, G, \mathcal{R})^{K(H, \alpha)}$ is a weak homotopy equivalence. Since $E(\Gamma, G, \mathcal{R})^{K(H, \alpha)}$ is weakly contractible, the same is true for $f^{*} E(\Gamma, G, \mathcal{R})^{K(H, \alpha)}$. Since $q$ is a principal $G$-bundle over the CW-complex $X$ with weakly contractible total space, it is a model for the universal principal $C_{G}(\alpha)-$ bundle and $X$ is a model for $B C_{G}(\alpha)$.

\section{Examples}

\subsection{Some special cases for $G, \Gamma$ and $\mathcal{R}$}

Example 14.1 (Trivial $\Gamma$ ) Suppose $\Gamma$ is trivial. Then there is only one family $\mathcal{R}$ of local representations consisting of $\{1\} \rightarrow G$, and the universal $\Gamma$-equivariant principal $G$-bundle with respect to the family of local representations $\mathcal{R}$ is the same as the universal principal $G$-bundle $E G \rightarrow B G$.

Example 14.2 (Trivial $G$ ) Suppose $G$ is trivial. Then a family of local representations is the same as a family $\mathcal{F}$ of subgroups of $\Gamma$, and the universal $\Gamma$-equivariant principal $G$-bundle with respect to the family of local representations $\mathcal{R}=\mathcal{F}$ is the identity $E_{\mathcal{F}}(\Gamma) \rightarrow E_{\mathcal{F}}(\Gamma)$.

Example 14.3 (Trivial local representations) Let $\mathcal{F}$ be a family of subgroups of $\Gamma$. Let $\mathcal{T} \mathcal{R}(\mathcal{F})$ be the system of local representations given by pairs $\left(H, \rho_{H}\right)$, where $H$ belongs to $\mathcal{F}$ and $\rho_{H}$ is the trivial representation. Then a $\Gamma$-equivariant principal $G$-bundle $q: E \rightarrow B$ satisfies $\mathcal{R}(p) \subseteq \mathcal{T} \mathcal{R}(\mathcal{F})$ if and only if all isotropy groups of $B$ belong to $\mathcal{F}$, the induced map $q / \Gamma: E / \Gamma \rightarrow B / \Gamma$ is a principal $G$-bundle, and $q$ is the pullback of $q / \Gamma$ with the projection $\operatorname{pr}: B \rightarrow B / \Gamma$. This follows from Theorem 9.1.

A model for the universal $\Gamma$-equivariant principal $G$-bundle associated to $\mathcal{T} \mathcal{R}(\mathcal{F})$ is

$$
\operatorname{id} \times p: E_{\mathcal{F}}(\Gamma) \times E G \rightarrow E_{\mathcal{F}}(\Gamma) \times B G,
$$

where $p: E G \rightarrow B G$ is the universal principal $G$-bundle, the left $\Gamma$ - and right $G-$ actions are given on the total space by $\gamma \cdot(x, e) \cdot g=(\gamma \cdot x, e \cdot g)$ and on the base space by $\gamma \cdot(x, b) \cdot g=(\gamma \cdot x, b)$.

Example 14.4 $(\mathcal{R}(\mathcal{F}))$ Consider a family $\mathcal{F}$ of subgroups of $\Gamma$. Define the associated family of local representations

$$
\mathcal{R}(\mathcal{F}):=\{(H, \alpha) \mid H \in \mathcal{F} \text { and } \alpha: H \rightarrow G \text { is any group homomorphism }\} ;
$$


in other words, $H$ runs through elements in $\mathcal{F}$ and $\alpha$ through all possible group homomorphisms.

Then a $\Gamma$-equivariant principle $G$-bundle $p: E \rightarrow B$ over the $\Gamma$-CW-complex $B$ satisfies $\mathcal{R}(p) \subseteq \mathcal{R}(\mathcal{F})$ if and only if all isotropy groups of $B$ belong to $\mathcal{F}$. If we suppose that $\mathcal{R}(\mathcal{F})$ satisfies Condition $(\mathrm{H})$ introduced in Definition 6.1, then in this situation the universal $\Gamma$-equivariant principal $G$-bundle with respect to $\mathcal{R}(\mathcal{F})$ classifies $\Gamma$-equivariant principal $G$-bundles over $\Gamma$-CW-complexes whose isotropy groups belong to $\mathcal{F}$.

If we choose $\mathcal{F}$ to be the family of compact subgroups and $\mathcal{R}(\mathcal{F})$ satisfies Condition $(\mathrm{H})$, these are precisely the $\Gamma$-equivariant principal $G$-bundles over proper $\Gamma-\mathrm{CW}-$ complexes.

Example 14.5 ( $n$-dimensional complex vector bundles with Hermitian metrics over proper $\Gamma-\mathrm{CW}-$ complexes) Let $\Gamma$ be a topological group. Assume that all compact subgroups of $\Gamma$ are Lie groups and $\Gamma$ is completely regular, eg $\Gamma$ is a Kac-Moody group, or that $\Gamma$ is locally compact, second countable and has finite covering dimension, eg is a Lie group. Let $G=\mathcal{U}(n)$ be the Lie group of unitary automorphisms of $\mathbb{C}^{n}$. Consider the family of representations

$\mathcal{R}:=\{(H, \alpha) \mid H$ is compact and $\alpha: H \rightarrow G$ is any group homomorphism $\}$,

which is the family $\mathcal{R}(\mathcal{C O M})$ of Example 14.4 associated to the family $\mathcal{C O M}$ of compact subgroups.

In this case $\operatorname{hom}(H, \mathcal{U}(n))$ is isomorphic to the space of unitary representations of $H$ on $\mathbb{C}^{n}$, and $\operatorname{hom}(H, \mathcal{U}(n)) / \mathcal{U}(n)$ is isomorphic to the set of isomorphism classes of $n-$ dimensional unitary $H$-representations. Denote by $V_{1}, V_{2}, \ldots, V_{k}, \ldots$ the irreducible unitary representations of $H$ and $d_{i}:=\operatorname{dim}_{\mathbb{C}} V_{i}$. The set of isomorphism classes unitary $n$-dimensional $H$-representations can be parametrized with the set of partitions of $n$ using the dimensions $d_{i}$, ie

$\operatorname{hom}(H, \mathcal{U}(n)) / \mathcal{U}(n) \cong\left\{\left(n_{1}, n_{2}, \ldots, n_{k}, \ldots\right) \mid n_{1} d_{1}+\cdots+n_{k} d_{k}+\cdots=n, n_{i} \geq 0\right\}$.

If the homomorphism $\alpha: H \rightarrow \mathcal{U}(n)$ induces the representation $V=\bigoplus_{i} V_{i}^{\oplus n_{i}}$, then the isotropy group is

$$
C_{\mathcal{U}(n)}(\alpha) \cong \prod_{i} \mathcal{U}\left(n_{i}\right)
$$

and therefore we have that

$$
B(\Gamma, \mathcal{U}(n), \mathcal{R})^{H} \cong \bigsqcup_{\left\{\left(n_{1}, n_{2}, \ldots, n_{k}, \ldots\right) \mid n_{1} d_{1}+\cdots+n_{k} d_{k}+\cdots=n, n_{i} \geq 0\right\}} \prod_{i} B \mathcal{U}\left(n_{i}\right) .
$$




\subsection{Compact abelian Lie group $G$}

Let $\Gamma$ be a topological group. Assume that all compact subgroups of $\Gamma$ are Lie groups and $\Gamma$ is completely regular, eg $\Gamma$ is a Kac-Moody group, or that $\Gamma$ is locally compact, second countable and has finite covering dimension, eg is a Lie group. Let $G$ be a compact abelian Lie group.

Consider the family of representations

$\mathcal{R}:=\{(H, \alpha) \mid H$ is compact and $\alpha: H \rightarrow G$ is any group homomorphism $\}$, which is the family $\mathcal{R}(\mathcal{C O M})$ of Example 14.4 associated to the family $\mathcal{C O M}$ of compact subgroups. We have

$$
\operatorname{hom}_{\mathcal{R}}(H, G)=\operatorname{hom}(H, G)
$$

and therefore we obtain from Theorem 13.1 a weak homotopy equivalence

$$
\bigsqcup_{\operatorname{hom}(H, G)} B G \simeq B(\Gamma, G, \mathcal{R})^{H} .
$$

Whenever $G=S^{1}$ we have that $\operatorname{hom}\left(H, S^{1}\right) \cong H^{2}(B H, \mathbb{Z})$ and $B S^{1} \simeq K(\mathbb{Z}, 2)$. Therefore we obtain a weak homotopy equivalence

$$
\bigsqcup_{H^{2}(B H, \mathbb{Z})} K(\mathbb{Z}, 2) \simeq B\left(\Gamma, S^{1}, \mathcal{R}\right)^{H} .
$$

Now make the stronger assumption that $\Gamma$ is a Lie group. Then for every compact subgroup $H \subseteq \Gamma$ the homogeneous space $\Gamma / H$ is a smooth manifold and hence a $\mathrm{CW}$-complex. This implies that $E \Gamma \times_{\Gamma} B(\Gamma, G, \mathcal{R})$ is a CW-complex. Because of Theorems 6.3 and 11.4 we have the universal $\Gamma$-equivariant $G$-bundle with respect to $\mathcal{R}$,

$$
p: E(\Gamma, G, \mathcal{R}) \rightarrow B(\Gamma, G, \mathcal{R}) .
$$

Applying the homotopy quotient with respect to the group $\Gamma$ we obtain a principal $G$-bundle over a CW-complex

$$
G \rightarrow E \Gamma \times_{\Gamma} E(\Gamma, G, \mathcal{R}) \rightarrow E \Gamma \times_{\Gamma} B(\Gamma, G, \mathcal{R})
$$

which can be classified by a map

$$
E \Gamma \times_{\Gamma} B(\Gamma, G, \mathcal{R}) \rightarrow B G .
$$

This map induces an adjoint map

$$
\psi: B(\Gamma, G, \mathcal{R}) \rightarrow \operatorname{map}(E \Gamma, B G)
$$


which is $\Gamma$-equivariant. In general the map $\psi$ is not a $\Gamma$-equivariant homotopy equivalence, but under the specific choices of $\Gamma, G$ and $\mathcal{R}$ above indeed they are.

Theorem 14.6 Let $\Gamma$ be a Lie group and let $G$ be a compact abelian Lie group. Put $\mathcal{R}=\mathcal{R}(\mathcal{C O M})$ for $\mathcal{C O M}$ the family of compact subgroups of $\Gamma$. Let $X$ be a proper $\Gamma-C W$ complex.

Then the map

$$
\operatorname{map}\left(\operatorname{id}_{X}, \psi\right): \operatorname{map}(X, B(\Gamma, G, \mathcal{R})) \rightarrow \operatorname{map}(X, \operatorname{map}(E \Gamma, B G)), \quad f \mapsto \psi \circ f,
$$

is a weak $\Gamma$-homotopy equivalence.

In particular we obtain bijections

$\left.\operatorname{Bundle}_{\Gamma, G, \mathcal{R}}(X) \cong[X, B(\Gamma, G, \mathcal{R}))\right]^{\Gamma} \cong[X, \operatorname{map}(E \Gamma, B G)]^{\Gamma}=\left[E \Gamma \times_{\Gamma} X, B G\right]$, and, when $G=S^{1}$,

$$
\text { Bundle }_{\Gamma, S^{1}, \mathcal{R}}(X) \cong H^{2}\left(E \Gamma \times_{\Gamma} X, \mathbb{Z}\right) .
$$

Proof For a compact subgroup $H$ of $\Gamma$, we claim that the induced map

$$
\psi^{H}: B(\Gamma, G, \mathcal{R})^{H} \rightarrow \operatorname{map}(E \Gamma, B G)^{H} \simeq \operatorname{map}(B H, B G)
$$

is a weak homotopy equivalence; the proof is based on the proofs and results of [20, Theorem 2 and Proposition 4]. From Theorem 13.1 we know that

$$
B(\Gamma, G, \mathcal{R})^{H} \cong \bigsqcup_{\alpha \in \operatorname{hom}(H, G)} B(\Gamma, G, \mathcal{R})_{\alpha}^{H},
$$

and since $G$ is abelian we know that

$$
E(\Gamma, G, \mathcal{R})^{K(H, \alpha)} \rightarrow B(\Gamma, G, \mathcal{R})_{\alpha}^{H}
$$

is a $G$-principal bundle. Therefore we have a commutative square of principal $G-$ bundles

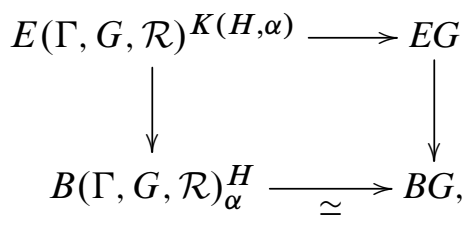


where the bottom arrow is a homotopy equivalence. This square induces another square of associated principal $G$-bundles

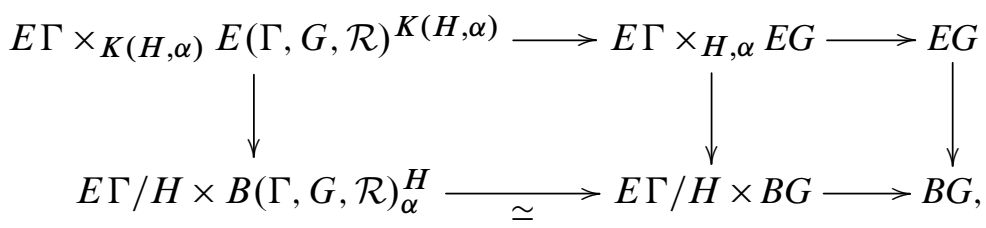

where on the upper left corner the group $K(H, \alpha)$ acts on $E \Gamma$ via the canonical isomorphism $H \cong K(H, \alpha)$, on the upper middle term the group $H$ acts on $E G$ via the homomorphism $\alpha: H \rightarrow G$ and the horizontal maps on the right-hand side are classifying maps. In [20, Proof of Theorem 2, page 173] it is shown that the adjoint map of the lower horizontal maps of diagram (14-1),

$$
B G \rightarrow \operatorname{map}(E \Gamma, E G)^{H} \simeq \operatorname{map}(B H, B G),
$$

is a weak equivalence on basepoint components; therefore the map

$$
\left.\psi^{H}\right|_{B(\Gamma, G, \mathcal{R})_{\alpha}^{H}}: B(\Gamma, G, \mathcal{R})_{\alpha}^{H} \rightarrow \operatorname{map}(E \Gamma, B G)^{H} \simeq \operatorname{map}(B H, B G)
$$

is also a weak equivalence of basepoint components. In [20, Proposition 4] it is shown that the map

$$
B: \operatorname{hom}(H, G) \rightarrow[B H, B G]
$$

given by the classifying space functor is an isomorphism, and since the bundle $E \Gamma \times_{H, \alpha} E G$ is constructed through the action defined by the homomorphism $\alpha$, then we conclude that the map

$$
\psi^{H}: B(\Gamma, G, \mathcal{R})^{H} \rightarrow \operatorname{map}(E \Gamma, B G)^{H} \simeq \operatorname{map}(B H, B G)
$$

is indeed a weak homotopy equivalence.

Hence for every proper $\Gamma-\mathrm{CW}-$ complex $Y$ the induced map

$$
\psi_{*}:[Y, B(\Gamma, G, \mathcal{R})]^{\Gamma} \rightarrow[Y, \operatorname{map}(E \Gamma, B G)]^{\Gamma}, \quad[f] \mapsto[\psi \circ f]
$$

is bijective; see [22, Proposition 2.3 on page 35].

In order to show that map $\left(\operatorname{id}_{X}, \psi\right)$ is a weak $\Gamma$-homotopy equivalence, it suffices to show for any $\Gamma-\mathrm{CW}-$ complex $Z$ that the induced map

$$
\begin{aligned}
& \operatorname{map}\left(\operatorname{id}_{X}, \psi\right)_{*}:[Z, \operatorname{map}(X, B(\Gamma, G, \mathcal{R}))]^{\Gamma} \rightarrow[Z, \operatorname{map}(X, \operatorname{map}(E \Gamma, B G))]^{\Gamma}, \\
& {[f] \mapsto\left[\operatorname{map}\left(\operatorname{id}_{X}, \psi\right) \circ f\right],}
\end{aligned}
$$


is bijective; see [22, Proposition 2.3 on page 35]. Because of the adjunctions appearing in Section A.4 and the identification $\pi_{0}\left(\operatorname{map}(A, B)^{\Gamma}\right)=[A, B]^{\Gamma}$ for $\Gamma$-spaces $A$ and $B$, this is equivalent to showing that adjoint map

$$
\psi_{*}:[X \times Z, B(\Gamma, G, \mathcal{R})]^{\Gamma} \rightarrow[X \times Z, \operatorname{map}(E \Gamma, B G)]^{\Gamma}, \quad[f] \mapsto[\psi \circ f]
$$

is bijective, where $\Gamma$ acts diagonally on $X \times Z$. Since $\Gamma$ is a Lie group and $X$ is a proper $\Gamma$-CW-complex, $X \times Y$ is $\Gamma$-homotopy equivalent to a proper $\Gamma$-CW-complex $Y$. Hence $\operatorname{map}\left(\operatorname{id}_{X}, \psi\right)$ is a weak $\Gamma$-homotopy equivalence because of the bijectivity of (14-2).

The other claims follow using Theorem 13.1.

\section{The case when $G=\mathcal{P} \mathcal{U}(\mathcal{H})$ is the projective unitary group}

Twisted versions of $K$-theory may be defined via a specific type of projective unitary bundles. The key point is that the $\operatorname{space} \operatorname{Fred}(\mathcal{H})$ of Fredholm operators on a separable Hilbert space $\mathcal{H}$ endowed with the norm topology, which itself has the homotopy type of $\mathbb{Z} \times B U$ (see Atiyah [3] and Jänich [13]), carries a conjugation action by the group $\mathcal{P U}(\mathcal{H})$ of projective unitary operators. Thus, to a pair $(X, P)$ of a $\mathrm{CW}$-complex $X$ together with a principal $\mathcal{P U}(\mathcal{H})$-bundle

$$
\mathcal{P U}(\mathcal{H}) \rightarrow P \rightarrow X
$$

over $X$, one can associate the twisted $K$-theory groups $K^{-i}(X, P)$ (see Atiyah and Segal [4]) defined as the homotopy groups

$$
K^{-i}(X, P):=\pi_{i}\left(\Gamma\left(P \times_{\mathcal{P U}(\mathcal{H})} \operatorname{Fred}(\mathcal{H})\right)\right)
$$

of the space of sections of the associated $\operatorname{Fred}(\mathcal{H})$-bundle

$$
\operatorname{Fred}(\mathcal{H}) \rightarrow P \times_{\mathcal{P U}(\mathcal{H})} \operatorname{Fred}(\mathcal{H}) \rightarrow X
$$

The equivariant version of the previous construction requires equivariant projective unitary bundles of a certain kind, and in order to construct their universal and classifying space we need to show that the group $\mathcal{P U}(\mathcal{H})$ satisfies items (i), (ii) and (iv) of Condition (H) introduced in Definition 6.1. In what follows, we will show that $\mathcal{P U}(\mathcal{H})$ satisfies items (i), (ii) and (iv) of Condition (H), whenever we consider homomorphisms $\alpha: H \rightarrow \mathcal{P U}(\mathcal{H})$ from finite groups $H$. 


\subsection{Existence of local cross sections for $H$ finite}

Let $\mathcal{U}(\mathcal{H})$ and $\mathcal{P U}(\mathcal{H})$ be respectively the unitary and projective unitary groups of a separable Hilbert space $\mathcal{H}$. The group $\mathcal{U}(\mathcal{H})$ is defined as

$$
\mathcal{U}(\mathcal{H}):=\left\{U \in \mathcal{B}(\mathcal{H}) \mid U U^{*}=U^{*} U=1\right\},
$$

where $\mathcal{B}(\mathcal{H})$ denotes the space of bounded operators on $\mathcal{H}$. Its center $Z(\mathcal{U}(\mathcal{H}))$ is $S^{1}$, and $\mathcal{P U}(\mathcal{H})$ is the quotient $\mathcal{U}(\mathcal{H}) / S^{1}$. We endow $\mathcal{U}(\mathcal{H})$ with the norm topology, ie a subbase for the topology is given by the sets

$$
B_{\epsilon}(T):=\{S \in \mathcal{U}(\mathcal{H}) \mid\|S-T\|<\epsilon\},
$$

where

$$
\|T\|:=\sup \{\|T x\| \mid x \in \mathcal{H} \text { such that }\|x\| \leq 1\} .
$$

Endow $\mathcal{P U}(\mathcal{H})$ with the quotient topology and note this topology can be recovered with the metric defined by the distance between the $S^{1}$-orbits, ie for $T, U \in \mathcal{P U}(\mathcal{H})$ define

$$
(T, U)_{\min }:=\min \{\|\tilde{T}-\tilde{U}\| \mid \tilde{T}, \tilde{U} \in \mathcal{U}(\mathcal{H}) \text { are lifts of } T, U \text { respectively }\} .
$$

With these topologies the groups $\mathcal{U}(\mathcal{H})$ and $\mathcal{P U}(\mathcal{H})$ become a topological groups. The short exact sequence of topological groups

$$
1 \rightarrow S^{1} \rightarrow \mathcal{U}(\mathcal{H}) \stackrel{p}{\rightarrow} \mathcal{P U}(\mathcal{H}) \rightarrow 1
$$

is an $S^{1}$-principal bundle (cf Simms [32]).

The group $\mathcal{U}(\mathcal{H})$ endowed with the norm topology is moreover a Banach Lie group; ${ }^{1}$ namely, $\mathcal{U}(\mathcal{H})$ is a Banach manifold whose structural maps are maps of Banach manifolds; see for instance [29, Example V.1.6, page 391]. It can be modeled locally by the vector space $\mathcal{A}$ of skew-adjoint operators

$$
\mathcal{A}:=\left\{L \in \mathcal{B}(\mathcal{H}) \mid L+L^{*}=0\right\}
$$

via the exponential map

$$
\text { exp: } \mathcal{A} \rightarrow \mathcal{U}(\mathcal{H}), \quad L \mapsto \exp (L) ;
$$

in this way we could think of the skew-adjoint operators as the tangent space of $\mathcal{U}(\mathcal{H})$ at the identity, $T_{1} \mathcal{U}(\mathcal{H})=\mathcal{A}$.

${ }^{1}$ A reference for the foundations of Banach manifolds may be found in Lang [17], and a reference on the properties the properties of Banach Lie groups and their Banach Lie algebras may be found in de la Harpe [10] and Neeb [29] and the references therein. 
This in particular implies that a base of open sets around $1 \in \mathcal{U}(\mathcal{H})$ may be obtained by the image of the exponential map of a base of open sets around $0 \in \mathcal{A}$. Denoting by $i \mathbb{R}$ the operators of the form $r \sqrt{-1} \cdot \mathrm{id}_{\mathcal{H}}$ for $r \in \mathbb{R}$, we obtain the diagram

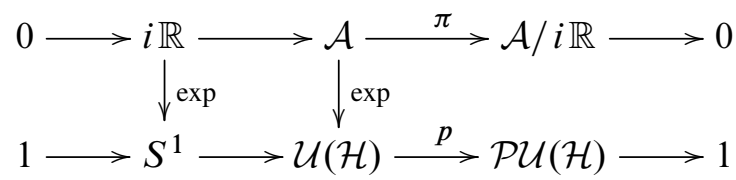

which in particular permits us to model locally $\mathcal{P U}(\mathcal{H})$ through any section

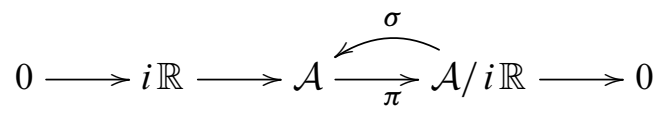

by the composition

$$
p \circ \exp \circ \sigma: \mathcal{A} / i \mathbb{R} \rightarrow \mathcal{P U}(\mathcal{H})
$$

making the following diagram commutative:

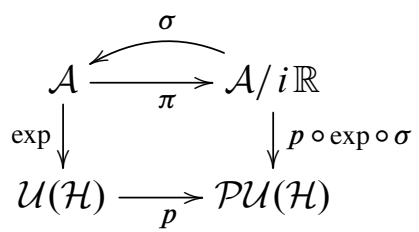

Now let $H$ be a finite group. Recall that $\operatorname{hom}(H, \mathcal{P U}(\mathcal{H}))$ obtains the subspace topology from its embedding into $\operatorname{map}(H, \mathcal{P U}(\mathcal{H}))$; see Section A.5. Since $\mathcal{P U}(\mathcal{H})$ is metric, this topology can also be defined with the supremum metric of (B-1).

Consider the conjugation action

$$
\mathcal{P U}(\mathcal{H}) \times \operatorname{hom}(H, \mathcal{P U}(\mathcal{H})) \rightarrow \operatorname{hom}(H, \mathcal{P U}(\mathcal{H})), \quad(g, \alpha) \mapsto g \alpha g^{-1}
$$

and for $\alpha \in \operatorname{hom}(H, \mathcal{P U}(\mathcal{H}))$ denote by

$$
\mathcal{P U}(\mathcal{H}) \cdot \alpha:=\left\{g \alpha g^{-1} \mid g \in \mathcal{P U}(\mathcal{H})\right\}
$$

the orbit of $\alpha$ under the conjugation action. We claim the following.

Theorem 15.1 For any finite group $H$ and any $\alpha \in \operatorname{hom}(H, \mathcal{P U}(\mathcal{H}))$, the projection

$$
\text { pr: } \mathcal{P U}(\mathcal{H}) \rightarrow \mathcal{P U}(H) / C_{\mathcal{P U}(\mathcal{H}))}(\alpha)
$$

is a principal $C_{\mathcal{P U}(\mathcal{H})}(\alpha)$-bundle and the canonical map

$$
\iota_{\alpha}: \mathcal{P U}(H) / C_{\mathcal{P U}(\mathcal{H})}(\alpha) \stackrel{\cong}{\rightarrow} \mathcal{P U}(H) \cdot \alpha
$$

is a homeomorphism. 
Its proof needs some preparation. For $\alpha: H \rightarrow \mathcal{P U}(\mathcal{H})$ denote by

$$
\tilde{H}:=\alpha^{*} \mathcal{U}(\mathcal{H})
$$

the central $S^{1}$-extension of $H$ defined by the pullback of $\alpha$ making the following diagram commutative:

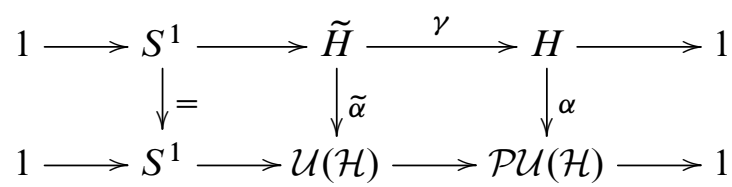

Denote the space of homomorphisms from $\tilde{H}$ to $\mathcal{U}(\mathcal{H})$ on which the kernel of the map $\widetilde{H} \stackrel{\gamma}{\rightarrow} H$ acts by multiplication by

$$
\operatorname{hom}_{S^{1}}(\tilde{H}, \mathcal{U}(\mathcal{H})):=\{f \in \operatorname{hom}(\tilde{H}, \mathcal{U}(\mathcal{H})) \mid f(x)=x \text { for all } x \in \operatorname{ker}(\gamma)\},
$$

and endow it with subspace topology $\operatorname{hom}_{S^{1}}(\tilde{H}, \mathcal{U}(\mathcal{H})) \subseteq \operatorname{hom}(\tilde{H}, \mathcal{U}(\mathcal{H}))$. (This is automatically compactly generated by the next result.)

Lemma 15.2 The space $\operatorname{hom}_{S^{1}}(\tilde{H}, \mathcal{U}(\mathcal{H}))$ is closed and open in $\operatorname{hom}(\tilde{H}, \mathcal{U}(\mathcal{H}))$.

Proof Consider the restriction map

$$
\begin{aligned}
A: \operatorname{hom}(\tilde{H}, \mathcal{U}(\mathcal{H})) & \rightarrow \operatorname{hom}\left(S^{1}, \mathcal{U}(\mathcal{H})\right), \\
f & \left.\mapsto f\right|_{\operatorname{ker}(\gamma)}
\end{aligned}
$$

and note that $\operatorname{hom}_{S^{1}}(\tilde{H}, \mathcal{U}(\mathcal{H}))=A^{-1}(\rho)$ where $\rho(\lambda)(x)=\lambda \cdot x$ for any $x \in \mathcal{H}$.

Take any other $\beta \in \operatorname{hom}\left(S^{1}, \mathcal{U}(\mathcal{H})\right)$ and since it defines a representation of $S^{1}$ different from $\rho$, there must exists an element $x \in \mathcal{H}$ of norm 1 and an integer $k \neq 1$ such that $\beta(\lambda) x=\lambda^{k} x$. We have then

$$
\|\rho, \beta\|_{\sup } \geq \sup _{\lambda \in S^{1}}\|\rho(\lambda) x-\beta(\lambda) x\|=\sup _{\lambda \in S^{1}}\left|1-\lambda^{k-1}\right|>1
$$

and therefore we can conclude that $\rho$ is an isolated point in $\operatorname{hom}\left(S^{1}, \mathcal{U}(\mathcal{H})\right)$. Hence $A^{-1}(\rho)$ is closed and open.

Define also the space of homomorphisms from $H$ to $\mathcal{P U}(\mathcal{H})$ which induce isomorphic central $S^{1}$-extensions of $H$ by

$\operatorname{hom}(H, \mathcal{P U}(\mathcal{H}))_{\tilde{H}}$

$$
:=\left\{\beta: H \rightarrow \mathcal{P U}(\mathcal{H}) \mid \beta^{*} \mathcal{U}(\mathcal{H}) \cong \widetilde{H} \text { as central } S^{1} \text {-extensions of } H\right\}
$$

and endow it with the subspace topology of $\operatorname{hom}(H, \mathcal{P U}(\mathcal{H}))$. (This is automatically compactly generated by the next result.) 
Lemma 15.3 The space $\operatorname{hom}(H, \mathcal{P U}(\mathcal{H}))_{\tilde{H}}$ is closed and open in $\operatorname{hom}(H, \mathcal{P U}(\mathcal{H}))$.

Proof The isomorphic classes of central extensions of a finite group are in one to one correspondence with the group $H^{2}\left(H, S^{1}\right)$. This cohomology group can be calculated by the continuous cohomology (see Segal [31, Example 1.4]) as the quotient of the topological groups

$$
\frac{\operatorname{ker}\left(\operatorname{map}\left(H^{2}, S^{1}\right) \stackrel{\delta}{\rightarrow} \operatorname{map}\left(H^{3}, S^{1}\right)\right)}{\operatorname{im}\left(\operatorname{map}\left(H, S^{1}\right) \stackrel{\delta}{\rightarrow} \operatorname{map}\left(H^{2}, S^{1}\right)\right)}
$$

where $\delta$ denotes the standard group cohomology differential. For a homomorphism $\alpha \in \operatorname{hom}(H, \mathcal{P U}(\mathcal{H}))$ define the $2-$ cocycle $\tilde{c}_{\alpha} \in \operatorname{map}\left(H^{2}, S^{1}\right)$ by

$$
\tilde{c}_{\alpha}(g, h):=\widetilde{\alpha(g)} \widetilde{\alpha(h)} \widetilde{\alpha(g h)}^{-1},
$$

where $\widetilde{\alpha(g)} \in \mathcal{U}(\mathcal{H})$ denotes fixed lifts of $\alpha(g)$ for all $g \in H$. If

$$
\bar{c}_{\alpha}(g, h):=\overline{\alpha(g)} \overline{\alpha(h)} \overline{\alpha(g h)}^{-1}
$$

denotes the 2-cocycle defined by a different choice of lifts, then, since $\overline{\alpha(g)} \widetilde{\alpha(g)}^{-1}$ is in the center of $\mathcal{U}(\mathcal{H})$ for all $g \in H$, the map $e \in \operatorname{map}\left(H, S^{1}\right)$,

$$
e(g):=\overline{\alpha(g)}_{\alpha(g)}{ }^{-1},
$$

satisfies the equation $\delta(e) \cdot \tilde{c}_{\alpha}=\bar{c}_{\alpha}$. Therefore we have the equality cohomology classes $\left[\tilde{c}_{\alpha}\right]=\left[\bar{c}_{\alpha}\right]$. Thus we can define a map

$$
\begin{aligned}
\rho: \operatorname{hom}(H, \mathcal{P U}(\mathcal{H})) & \rightarrow H^{2}\left(H, S^{1}\right), \\
\alpha & \mapsto\left[\tilde{c}_{\alpha}\right] .
\end{aligned}
$$

Next we prove that it is continuous. Since $H$ is finite and $\mathcal{U}(\mathcal{H}) \rightarrow \mathcal{P U}(\mathcal{H})$ is a principal $S^{1}$-bundle, we can define a small neighborhood $V$ of $\alpha$ in $\operatorname{hom}(H, \mathcal{P U}(\mathcal{H}))$ such that for every $h$ there exists a continuous map $V \rightarrow \mathcal{U}, \beta \mapsto \widetilde{\beta(h)}$ whose value for $\beta=\alpha$ is the given lift $\widetilde{\alpha(h)}$ and whose composite with the projection $\mathcal{U}(H) \rightarrow \mathcal{P U}(H)$ is the evaluation map $\beta \mapsto \beta(h)$. Now we can define a continuous map

$$
\begin{aligned}
V \rightarrow \operatorname{map}\left(H^{2}, S^{1}\right)_{0}:=\operatorname{ker}\left(\operatorname{map}\left(H^{2}, S^{1}\right) \stackrel{\delta}{\rightarrow} \operatorname{map}\left(H^{3}, S^{1}\right)\right), \\
\beta \mapsto \widetilde{c}_{\beta}:=\widetilde{\beta(g)} \widetilde{\beta(h)} \widetilde{\beta(g h)}-1 .
\end{aligned}
$$

Since the quotient map

$$
\operatorname{map}\left(H^{2}, S^{1}\right)_{0} \rightarrow \operatorname{map}\left(H^{2}, S^{1}\right)_{0} / \operatorname{Im}(\delta)=H^{2}\left(H, S^{1}\right)
$$


is continuous, and $H^{2}\left(H, S^{1}\right)$ is finite, we conclude that the map $\rho$ is continuous. Any 2-cocycle $\tilde{c}_{\alpha}$ defines a $S^{1}$-central extension $H \times \widetilde{c}_{\alpha} S^{1}$ of $H$, and it is classical result in group theory that $\widetilde{H}$ and $H \times_{\widetilde{c}_{\alpha}} S^{1}$ are isomorphic as central extensions of $H$.

Therefore $\operatorname{hom}(H, \mathcal{P U}(\mathcal{H}))_{\tilde{H}}=\rho^{-1}\left(\left[\tilde{c}_{\alpha}\right]\right)$ and hence we have that it is open and closed in $\operatorname{hom}(H, \mathcal{P U}(\mathcal{H}))$.

Before we proceed with the study of the canonical map $\phi: \operatorname{hom}_{S^{1}}(\tilde{H}, \mathcal{U}(\mathcal{H})) \rightarrow$ $\operatorname{hom}(H, \mathcal{P U}(\mathcal{H}))_{\tilde{H}}$, we will make use of Banach Lie group structure of $\mathcal{U}(\mathcal{H})$ to prove the following lemma.

Lemma 15.4 For $n>1$ let $\mathcal{U}(\mathcal{H})_{n}:=\left\{U \in \mathcal{U}(\mathcal{H}): U^{n}=1\right\}$ and let $\mathcal{P U}(\mathcal{H})_{n}:=$ $\left\{U \in \mathcal{P U}(\mathcal{H}) \mid U^{n}=1\right\}$. Then the projection map

$$
\left.p\right|_{\mathcal{U}(\mathcal{H})_{n}}: \mathcal{U}(\mathcal{H})_{n} \rightarrow \mathcal{P U}(\mathcal{H})_{n}
$$

is a local homeomorphism.

Proof The subspace $\mathcal{U}(\mathcal{H})_{n}$ is a Banach submanifold of $\mathcal{U}(\mathcal{H})$ since it is the inverse image $f^{-1}(\{1\})=\mathcal{U}(\mathcal{H})_{n}$ for the function

$$
f: \mathcal{U}(\mathcal{H}) \rightarrow \mathcal{U}(\mathcal{H}), \quad U \mapsto U^{n}
$$

that is analytical. Take $U \in \mathcal{U}(\mathcal{H})_{n}$ and consider the isomorphism of tangent spaces

$$
R_{U^{-1}}: T_{U} \mathcal{U}(\mathcal{H}) \stackrel{\cong}{\rightrightarrows} T_{1} \mathcal{U}(\mathcal{H}), \quad L \mapsto L U^{-1} .
$$

Since the tangent space of $U \in \mathcal{U}(\mathcal{H})_{n}$ at $U$ is

$$
T_{U} \mathcal{U}(\mathcal{H})_{n}=\left\{L \in \mathcal{B}(\mathcal{H}) \mid L U^{*}+U L^{*}=0 \text { and } \sum_{k=0}^{n-1} U^{k} L U^{n-k-1}=0\right\},
$$

its image under $R_{U^{-1}}$ becomes

$$
R_{U^{-1}} T_{U} \mathcal{U}(\mathcal{H})_{n}=\left\{A+A^{*}=0 \text { and } \sum_{k=0}^{n-1} U^{k} A U^{n-k}=0\right\} .
$$

The vector space $i \mathbb{R}$ of diagram (15-1) is not included in $R_{U^{-1}} T_{U} \mathcal{U}(\mathcal{H})_{n}$ since for $A=r \sqrt{-1}$ with $r \neq 0$ the sum $\sum_{k=0}^{n-1} U^{k} A U^{n-k}=n r \sqrt{-1} \neq 0$. Therefore the vector space $R_{U^{-1}} T_{U} \mathcal{U}(\mathcal{H})_{n}$ maps isomorphically to $\pi\left(R_{U^{-1}} T_{U} \mathcal{U}(\mathcal{H})_{n}\right)$ under the map $\pi$ of diagram (15-1). By the inverse function theorem for Banach manifolds [17, Theorem 1, Section I.5, page 13] the map $p$ restricted to $\mathcal{U}(\mathcal{H})_{n} \cdot U^{-1}$ has a local inverse 
around 1 , and hence the map $\left.p\right|_{\mathcal{U}(\mathcal{H})_{n}}: \mathcal{U}(\mathcal{H})_{n} \rightarrow \mathcal{P U}(\mathcal{H})_{n}$ has a local inverse around a neighborhood of $U$ in $\mathcal{U}(\mathcal{H})_{n}$.

\section{Proposition 15.5 The canonical map}

$$
\begin{aligned}
\phi: \operatorname{hom}_{S^{1}}(\tilde{H}, \mathcal{U}(\mathcal{H})) & \rightarrow \operatorname{hom}(H, \mathcal{P U}(\mathcal{H}))_{\tilde{H}}, \\
f: \tilde{H} \rightarrow \mathcal{U}(\mathcal{H}) & \mapsto f / S^{1}: \tilde{H} / S^{1} \rightarrow \mathcal{U}(\mathcal{H}) / S^{1},
\end{aligned}
$$

is a principal $\operatorname{hom}\left(H, S^{1}\right)$-bundle, and since $\operatorname{hom}\left(H, S^{1}\right)$ is finite, it is also a local homeomorphism.

Proof The map $\phi: \operatorname{hom}_{S^{1}}(\tilde{H}, \mathcal{U}(\mathcal{H})) \rightarrow \operatorname{hom}(H, \mathcal{P U}(\mathcal{H}))_{\tilde{H}}$ is continuous because of Lemma A.4. One easily checks that it is surjective.

Define the $\operatorname{hom}\left(H, S^{1}\right)$-action on $\operatorname{hom}_{S^{1}}(\tilde{H}, \mathcal{U}(\mathcal{H}))$ by the map

$$
\operatorname{hom}\left(H, S^{1}\right) \times \operatorname{hom}_{S^{1}}(\tilde{H}, \mathcal{U}(\mathcal{H})) \rightarrow \operatorname{hom}_{S^{1}}(\tilde{H}, \mathcal{U}(\mathcal{H})), \quad(r, f) \mapsto r \cdot f,
$$

where $(r \cdot f)(\tilde{h}):=r(\gamma(\tilde{h})) \cdot f(\tilde{h})$ for all $\tilde{h} \in \tilde{H}$. One easily checks that the action is free and that $\phi$ and $\phi(f)=\phi(r \cdot f)$ holds for all $r \in \operatorname{hom}\left(H, S^{1}\right)$ and $f \in \operatorname{hom}_{S^{1}}(\tilde{H}, \mathcal{U}(\mathcal{H}))$. Hence $\phi$ induces a surjective map

$$
\bar{\phi}: \operatorname{hom}_{S^{1}}(\tilde{H}, \mathcal{U}(\mathcal{H})) / \operatorname{hom}\left(H, S^{1}\right) \rightarrow \operatorname{hom}_{S^{1}}(\tilde{H}, \mathcal{U}(\mathcal{H})) .
$$

Next we show that $\bar{\phi}$ is injective and therefore that $\phi$ is locally bijective. Consider $f, g \in \operatorname{hom}_{S^{1}}(\tilde{H}, \mathcal{U}(\mathcal{H}))$ with $\phi(f)=\phi(g)$. For all $h \in H$, take any lift $\tilde{h} \in \tilde{H}$ such that $\gamma(\tilde{h})=h$ and define the element $r(h) \in S^{1}$ by the equation $f(\tilde{h})=r(h) g(\tilde{h})$. Note that $r(h)$ does not depend on the choice of lift and therefore we obtain a map $r: H \rightarrow S^{1}$. For $h, k \in H$ with respective lifts $\widetilde{h}, \tilde{k} \in \widetilde{H}$ one has

$$
r(h k) g(\tilde{h} \tilde{k})=f(\tilde{h} \tilde{k})=r(h) g(\tilde{h}) r(k) g(\tilde{k})=r(h) r(k) g(\tilde{h} \tilde{k}),
$$

and therefore we see that $r \in \operatorname{hom}\left(H, S^{1}\right)$ and $r \cdot f=g$.

We are left with showing that $\phi$ is a local homeomorphism.

For $h \in H$ denote by $|h|$ its order and by $\langle h\rangle$ the cyclic group it generates. Since all central $S^{1}$-extensions of cyclic groups are trivializable, the restriction of $\widetilde{H}$ to $\langle h\rangle$ must be isomorphic to $S^{1} \times\langle h\rangle$; therefore we can choose a lift $\tilde{h} \in \tilde{H}$ for each $h \in H$ such that $\tilde{h}$ generates a cyclic group of order $|h|$. 
Define the restriction maps

$$
\begin{array}{cc}
\Psi: \operatorname{hom}_{S^{1}}(\tilde{H}, \mathcal{U}(\mathcal{H})) \rightarrow \prod_{h \in H} \operatorname{hom}(\langle\tilde{h}\rangle, \mathcal{U}(\mathcal{H})),\left.\quad \tilde{\alpha} \mapsto \prod_{h \in H} \tilde{\alpha}\right|_{\langle\tilde{h}\rangle}, \\
\psi: \operatorname{hom}(H, \mathcal{P U}(\mathcal{H}))_{\tilde{H}} \rightarrow \prod_{h \in H} \operatorname{hom}(\langle h\rangle, \mathcal{P U}(\mathcal{H})),\left.\quad \alpha \mapsto \prod_{h \in H} \alpha\right|_{\langle h\rangle},
\end{array}
$$

and note that both maps $\Psi$ and $\psi$ are injective. We claim moreover that the map $\Psi$ induces a homeomorphism onto its image. The proof is as follows: For $\epsilon>0$ and $\tilde{\alpha} \in \operatorname{hom}_{S^{1}}(\tilde{H}, \mathcal{U}(\mathcal{H}))$, consider the open ball of radius $\epsilon$ defined by the supremum metric of (B-1),

$$
B_{\epsilon}(\widetilde{\alpha}):=\left\{f \in \operatorname{hom}_{S^{1}}(\tilde{H}, \mathcal{U}(\mathcal{H})) \mid\|\widetilde{\alpha}(g)-f(g)\|<\epsilon \text { for all } g \in \tilde{H}\right\},
$$

and note that this open ball can also be defined as

$$
B_{\epsilon}(\tilde{\alpha}):=\left\{f \in \operatorname{hom}_{S^{1}}(\tilde{H}, \mathcal{U}(\mathcal{H})) \mid\|\widetilde{\alpha}(\tilde{h})-f(\tilde{h})\|<\epsilon \text { for all } h \in H\right\},
$$

since for all $\lambda \in S^{1}$ we have that

$$
\|\widetilde{\alpha}(\tilde{h})-f(\tilde{h})\|=\|\widetilde{\alpha}(\lambda \cdot \tilde{h})-f(\lambda \cdot \tilde{h})\| .
$$

For the restricted homomorphisms $\left.\widetilde{\alpha}\right|_{\langle\widetilde{h}\rangle}$ we can also consider the open balls of radius $\epsilon$

$$
B_{\epsilon}\left(\left.\widetilde{\alpha}\right|_{\langle\tilde{h}\rangle}\right):=\{f \in \operatorname{hom}(\langle\widetilde{h}\rangle, \mathcal{U}(\mathcal{H})) \mid\|\widetilde{\alpha}(g)-f(g)\|<\epsilon \text { for all } g \in\langle\widetilde{h}\rangle\},
$$

and therefore we get the following equality of sets:

$$
\Psi\left(B_{\epsilon}(\widetilde{\alpha})\right)=\left(\prod_{h \in H} B_{\epsilon}\left(\left.\widetilde{\alpha}\right|_{\langle\widetilde{h}\rangle}\right)\right) \cap \Psi\left(\operatorname{hom}_{S^{1}}(\tilde{H}, \mathcal{U}(\mathcal{H}))\right)
$$

This implies that the map $\Psi$ induces an open map onto its image, and since it is injective and continuous, it induces a homeomorphism onto its image.

Denoting by $\phi_{h}: \operatorname{hom}(\langle\tilde{h}\rangle, \mathcal{U}(\mathcal{H})) \rightarrow \operatorname{hom}(\langle h\rangle, \mathcal{P U}(\mathcal{H}))$ the canonical map defined by $\phi_{h}(\gamma)(h)=\gamma(\widetilde{h})$, we obtain the following commutative diagram:

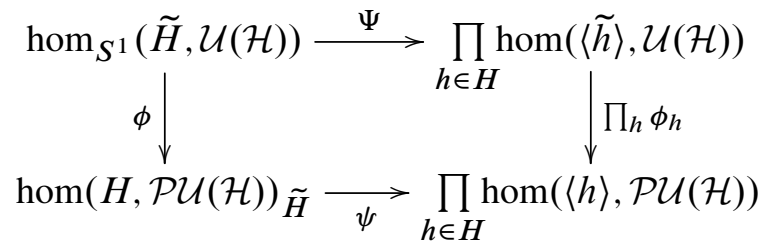

Since there are canonical homeomorphisms

$$
\operatorname{hom}(\langle\tilde{h}\rangle, \mathcal{U}(\mathcal{H})) \stackrel{\cong}{\rightarrow} \mathcal{U}(\mathcal{H}))_{|h|}, \quad f \mapsto f(\tilde{h}),
$$


by Lemma 15.4 we know that there must exist $\epsilon$ such that the maps

$$
\phi_{h}: B_{\epsilon}\left(\left.\tilde{\alpha}\right|_{\langle\widetilde{h}\rangle}\right) \rightarrow \phi_{h}\left(B_{\epsilon}\left(\left.\tilde{\alpha}\right|_{\langle\tilde{h}\rangle}\right)\right)
$$

are homeomorphisms for all $h \in H$; denote by

$$
\tau_{h}: \phi_{h}\left(B_{\epsilon}\left(\left.\widetilde{\alpha}\right|_{\langle\widetilde{h}\rangle}\right)\right) \rightarrow B_{\epsilon}\left(\left.\widetilde{\alpha}\right|_{\langle\widetilde{h}\rangle}\right)
$$

these inverse maps. Restricting to the open subset $B_{\epsilon}(\widetilde{\alpha})$ of the top left corner of the diagram (15-3), we obtain the following diagram:

$$
\begin{gathered}
B_{\epsilon}(\widetilde{\alpha}) \stackrel{\left(\left.\Psi\right|_{B_{\epsilon}(\widetilde{\alpha})}\right)^{-1}}{\cong} \Psi\left(B_{\epsilon}(\widetilde{\alpha})\right) \\
\phi \downarrow \\
\left.\quad \cong \uparrow\left(\prod_{h} \tau_{h}\right)\right|_{\psi\left(\phi\left(B_{\epsilon}(\widetilde{\alpha})\right)\right)} \\
\phi\left(B_{\epsilon}(\widetilde{\alpha})\right) \underset{\psi}{\longrightarrow} \psi\left(\phi\left(B_{\epsilon}(\widetilde{\alpha})\right)\right),
\end{gathered}
$$

where the right-hand side vertical arrow is the homeomorphism that the maps $\tau_{h}$ induce once restricted to the open set

$$
\psi\left(\phi\left(B_{\epsilon}(\widetilde{\alpha})\right)\right)=\left(\prod_{h} \phi_{h}\left(B_{\epsilon}\left(\left.\widetilde{\alpha}\right|_{\langle\widetilde{h}}\right)\right)\right) \cap\left(\prod_{h} \phi_{h}\right) \Psi\left(B_{\epsilon}(\widetilde{\alpha})\right),
$$

and the upper horizontal arrow is the inverse of $\Psi$ restricted to $B_{\epsilon}(\widetilde{\alpha})$.

Since the map $\psi$ is injective, we can define the map

$$
\left.\left(\left.\Psi\right|_{B_{\epsilon}(\widetilde{\alpha})}\right)^{-1} \circ\left(\prod_{h} \tau_{h}\right)\right|_{\psi\left(\phi\left(B_{\epsilon}(\widetilde{\alpha})\right)\right)} \circ \psi: \phi\left(B_{\epsilon}(\widetilde{\alpha})\right) \rightarrow B_{\epsilon}(\widetilde{\alpha}),
$$

which clearly is the inverse map of $\phi$ once it is restricted to $B_{\epsilon}(\widetilde{\alpha})$. This proves that $\phi$ is a local homeomorphism.

The conjugation action of $\mathcal{U}(\mathcal{H})$ on $\operatorname{hom}_{S^{1}}(\tilde{H}, \mathcal{U}(\mathcal{H}))$ factors through the projection $\mathcal{U}(\mathcal{H}) \rightarrow \mathcal{P U}(\mathcal{H})$ to an action

$$
\mathcal{P U}(\mathcal{H})) \times \operatorname{hom}_{S^{1}}(\tilde{H}, \mathcal{U}(\mathcal{H})) \rightarrow \operatorname{hom}_{S^{1}}(\tilde{H}, \mathcal{U}(\mathcal{H})),
$$

since the conjugation action restricted to the center of $\mathcal{U}(\mathcal{H})$ is trivial.

Lemma 15.6 Let $\alpha: H \rightarrow \mathcal{P U}(\mathcal{H})$ and $\tilde{\alpha}: \tilde{H} \rightarrow \mathcal{U}(\mathcal{H})$ be as in diagram (15-2). Then the existence of a local cross section for the conjugation map $\mathcal{U}(\mathcal{H}) \rightarrow \mathcal{U}(\mathcal{H}) \cdot \widetilde{\alpha}$ implies the existence of a local cross section of the conjugation map $\mathcal{P U}(\mathcal{H}) \rightarrow \mathcal{P U}(\mathcal{H}) \cdot \alpha$. 
Proof This follows from the commutativity of the diagram

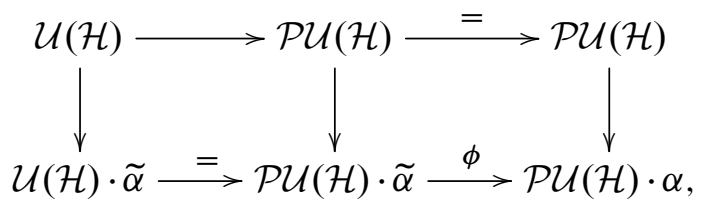

where the vertical arrows are defined by conjugation on $\widetilde{\alpha}$ and $\alpha$ respectively, and the conclusion of Proposition 15.5 that the map $\phi$ is a local homeomorphism.

Lemma 15.7 For $\tilde{\alpha} \in \operatorname{hom}_{S^{1}}(\tilde{H}, \mathcal{U}(\mathcal{H}))$ the map induced by the conjugation action

$$
\mathcal{U}(\mathcal{H}) \rightarrow \mathcal{U}(\mathcal{H}) \cdot \widetilde{\alpha}, \quad g \mapsto g \widetilde{\alpha} g^{-1}
$$

has a local cross section.

Proof For $\beta, \gamma \in \operatorname{hom}_{S^{1}}(\tilde{H}, \mathcal{U}(\mathcal{H}))$ let

$$
T_{\beta, \gamma}:=\int_{\tilde{H}} \beta(\tilde{h}) \circ \gamma\left(\tilde{h}^{-1}\right) d \tilde{h} \in \mathcal{U}(\mathcal{H})
$$

be the intertwining operator between $\beta$ and $\gamma$ in Gaal [8, VII.1.Proposition 21], where $d \tilde{h}$ is the normalized Haar measure on $\tilde{H}$. We have

$$
\begin{aligned}
\beta \circ T_{\beta, \gamma} & =T_{\beta, \gamma} \circ \gamma, \\
T_{\beta, \gamma}^{*} & =T_{\gamma, \beta},
\end{aligned}
$$

since for all $a \in \tilde{H}$ one has

$$
\begin{aligned}
T_{\beta, \gamma} \circ \gamma(a)=\int_{\widetilde{H}} \beta(\tilde{h}) \circ \gamma\left(\tilde{h}^{-1} a\right) d \tilde{h} & =\int_{\widetilde{H}} \beta(a \tilde{h}) \circ \gamma\left(\tilde{h}^{-1}\right) d \tilde{h}=\beta(a) \circ T_{\beta, \gamma}, \\
T_{\beta, \gamma}^{*}=\int_{\widetilde{H}}\left(\beta(\widetilde{h}) \circ \gamma\left(\tilde{h}^{-1}\right)\right)^{*} d \tilde{h} & =\int_{\widetilde{H}} \gamma\left(\tilde{h}^{-1}\right)^{*} \cdot \beta(\widetilde{h})^{*} d \tilde{h} \\
& =\int_{\widetilde{H}} \gamma\left(\tilde{h}^{-1}\right)^{-1} \cdot \beta(\tilde{h})^{-1} d \tilde{h} \\
& =\int_{\widetilde{H}} \gamma(\tilde{h}) \cdot \beta\left(\tilde{h}^{-1}\right) d \tilde{h}=T_{\gamma, \beta} .
\end{aligned}
$$

Notice that since $\beta$ and $\gamma$ belong to $\operatorname{hom}_{S^{1}}(\tilde{H}, \mathcal{U}(\mathcal{H}))$, then the intertwiner $T_{\beta, \gamma}$ can also be defined by the finite sum

$$
T_{\beta, \gamma}:=\frac{1}{|H|} \cdot \sum_{h \in H} \beta(\tilde{h}) \circ \gamma\left(\tilde{h}^{-1}\right),
$$


where $\tilde{h}$ is any fixed choice of lift of $h$ in $\tilde{H}$. We can choose an open neighborhood of $\tilde{\alpha}$ :

$$
V:=\left\{\beta \in \operatorname{hom}_{S^{1}}(\tilde{H}, \mathcal{U}(\mathcal{H})) \mid\|\alpha(\tilde{h})-\beta(\tilde{h})\|<\frac{1}{|H|} \text { for all } h \in H\right\} .
$$

The triangle inequality implies that for all $\beta$ in $V$ we have $\left\|1-T_{\beta, \widetilde{\alpha}}\right\|<1$ and therefore the operator $T_{\beta, \tilde{\alpha}}$ is invertible. Hence we get a map

$$
\tau: V \rightarrow G L(\mathcal{H}), \quad \beta \mapsto T_{\beta, \tilde{\alpha}},
$$

whose continuity follows from the finite sum definition of $T_{\beta, \tilde{\alpha}}$ of (15-4). It satisfies for every $\beta \in V$,

$$
\begin{aligned}
\tau(\beta) \circ \tilde{\alpha} \circ \tau(\beta)^{-1} & =\beta, \\
\tau(\beta)^{*} \circ \beta & =\widetilde{\alpha} \circ \tau(\beta)^{*} .
\end{aligned}
$$

Composing the map $\tau$ with the retraction

$$
\rho: G L(\mathcal{H}) \rightarrow \mathcal{U}(\mathcal{H}), \quad T \mapsto T{\sqrt{\left(T^{*} T\right)}}^{-1}
$$

defined by Kuiper in [16, Chapter 4], we obtain a continuous map

$$
\sigma:=\rho \circ \tau: V \rightarrow \mathcal{U}(\mathcal{H})
$$

from an open neighborhood $V$ of $\widetilde{\alpha}$ in $\operatorname{hom}_{S^{1}}(\tilde{H}, \mathcal{U}(\mathcal{H}))$ to $\mathcal{U}(\mathcal{H})$ with

$$
\sigma(\beta) \circ \tilde{\alpha} \circ \sigma(\beta))^{-1}=\beta .
$$

Since the action of $\mathcal{U}(\mathcal{H})$ is transitive on $\mathcal{U}(\mathcal{H}) \cdot \widetilde{\alpha}$, the translations of the previously defined local cross section define local cross sections around any point in the orbit space.

Corollary 15.8 The space $\operatorname{hom}_{S^{1}}(\tilde{H}, \mathcal{U}(\mathcal{H}))$ is homeomorphic to the disjoint union of its orbits under the conjugation action of $\mathcal{U}(\mathcal{H})$. Hence each orbit $\mathcal{U}(\mathcal{H}) \cdot \widetilde{\alpha}$ is open and closed in $\operatorname{hom}_{S^{1}}(\tilde{H}, \mathcal{U}(\mathcal{H}))$.

Proof From the proof of Lemma 15.7 we know that for $\alpha, \beta \in \operatorname{hom}_{S^{1}}(\tilde{H}, \mathcal{U}(\mathcal{H}))$ lying in different orbits there must exist $h \in H$ such that for all lifts $\tilde{h}$,

$$
\|\alpha(\tilde{h})-\beta(\tilde{h})\| \geq \frac{1}{|H|},
$$

and therefore the distance between $\alpha$ and $\beta$ is greater or equal than $1 /|H|$. This implies that different orbits are separated by at least a distance of $1 /|H|$ and therefore $\operatorname{hom}_{S^{1}}(\tilde{H}, \mathcal{U}(\mathcal{H}))$ is homeomorphic to the disjoint union of its orbits under the conjugation action of $\mathcal{U}(\mathcal{H})$. 
Proof of Theorem 15.1 Lemmas 15.6 and 15.7 imply the existence of local cross sections for the map

$$
\mathcal{P U}(\mathcal{H}) \rightarrow \mathcal{P U}(\mathcal{H}) \cdot \alpha, \quad g \mapsto g \alpha g^{-1} .
$$

This map is the composite of the projection pr: $\mathcal{P U}(\mathcal{H}) \rightarrow \mathcal{P U}(H) / C_{\mathcal{P U}(\mathcal{H}))}(\alpha)$ with the bijective continuous map $\iota_{\alpha}: \mathcal{P U}(H) / C_{\mathcal{P U}(\mathcal{H}))}(\alpha) \rightarrow \mathcal{P U}(H) \cdot \alpha$. This implies that $\iota_{\alpha}$ has local cross sections and hence is a homeomorphism. We conclude that pr: $\mathcal{P U}(\mathcal{H}) \rightarrow \mathcal{P U}(H) / C_{\mathcal{P U}(\mathcal{H}))}(\alpha)$ has local cross sections and hence is a principal $C_{\mathcal{P U}(\mathcal{H}))}(\alpha)$-bundle by Remark 4.3. This finishes the proof of Theorem 15.1.

Proposition 15.9 Let $H$ be a finite group and $\alpha \in \operatorname{hom}(H, \mathcal{P U}(\mathcal{H}))$. Then (i), (ii) and (iv) of Condition $(H)$ are satisfied:

(i) The path component of $\alpha$ in $\operatorname{hom}(H, \mathcal{P U}(\mathcal{H}))$ is contained in the orbit $\mathcal{P U}(\mathcal{H}) \cdot \alpha$.

(ii) The canonical map $\mathcal{P U}(\mathcal{H}) \rightarrow \mathcal{P U}(\mathcal{H}) / C_{\mathcal{P U}(\mathcal{H})}(\alpha)$ is a $C_{\mathcal{P U}(\mathcal{H})}(\alpha)$-principal bundle.

(iv) The canonical map

$$
\iota_{\alpha}: \mathcal{P U}(\mathcal{H}) / C_{\mathcal{P U}(\mathcal{H})}(\alpha) \rightarrow \operatorname{hom}(H, \mathcal{P U}(\mathcal{H})), \quad g C_{\mathcal{P U}(\mathcal{H})}(\alpha) \mapsto g \alpha g^{-1}
$$

is a homeomorphism onto its image.

Proof Consider the commutative diagram

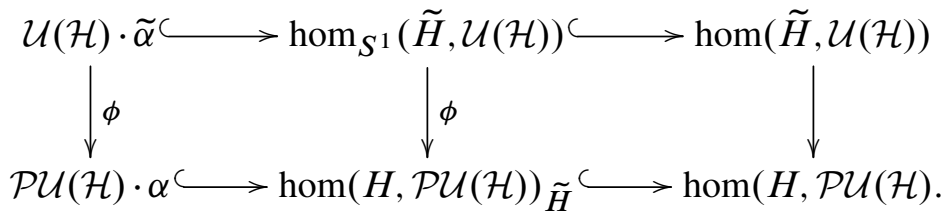

By Lemma 15.3 we know $\operatorname{hom}(H, \mathcal{P U}(\mathcal{H}))_{\tilde{H}}$ is open and closed in $\operatorname{hom}(H, \mathcal{P U}(\mathcal{H})$, by Proposition 15.5 we know that the map $\phi$ is a principal $\operatorname{hom}\left(H, S^{1}\right)$-bundle and by Corollary 15.8 we know that $\mathcal{U}(\mathcal{H}) \cdot \widetilde{\alpha}$ is both open and closed in $\operatorname{hom}_{S^{1}}(\tilde{H}, \mathcal{U}(\mathcal{H}))$. This implies that $\mathcal{P U}(\mathcal{H}) \cdot \alpha$ is open and closed in $\operatorname{hom}(H, \mathcal{P U}(\mathcal{H})$; this proves (i).

The other two conditions (ii) and (iii) have already been proved in Theorem 15.1.

\subsection{Almost free $\Gamma$-equivariant stable projective unitary bundles}

Let $\Gamma$ be a Hausdorff topological group and consider the family of all finite subgroups $\mathcal{F} \mathcal{I} \mathcal{N}(\Gamma)$ of $\Gamma$. Let $\mathcal{R}=\mathcal{R}(\mathcal{F} \mathcal{I N}(\Gamma))$ be the associated family of local representations $\mathcal{R}=\{(H, \alpha) \mid H \in \mathcal{F} \mathcal{I N}(\Gamma)$ and $\alpha: H \rightarrow \mathcal{P U}(\mathcal{H})$ is any group homomorphism $\}$.

A $\Gamma-\mathrm{CW}-$ complex $X$ is called almost free if all its isotropy groups are finite. 
Theorem 15.10 (Universal $\Gamma$-equivariant projective unitary bundle for almost free $\Gamma-\mathrm{CW}-\mathrm{complexes)}$ Let $\Gamma$ be a Hausdorff topological group. Then the bundle

$$
\mathcal{P U}(\mathcal{H}) \rightarrow E(\Gamma, \mathcal{P U}(\mathcal{H}), \mathcal{R}) \stackrel{p}{\rightarrow} B(\Gamma, \mathcal{P U}(\mathcal{H}), \mathcal{R})
$$

is a universal $\Gamma$-equivariant projective unitary bundle for almost free $\Gamma-C W-$ complexes.

Proof The result follows from Theorem 11.4 since items (i), (ii) and (iv) of Condition $(\mathrm{H})$ were proved in Proposition 15.9 and item (iii) follows from the fact that $\Gamma$ is Hausdorff and the subgroups $H \in \mathcal{F} \mathcal{I} \mathcal{N}(\Gamma)$ are finite.

Certainly one can associate a $\operatorname{Fred}(\mathcal{H})$-bundle to any $\Gamma$-equivariant projective unitary bundle and one can take the homotopy groups of its $\Gamma$-equivariant sections. This procedure applied to some $\Gamma$-equivariant projective unitary bundles may fail to produce the expected twisted equivariant $K$-theory groups, let us see why: For a finite subgroup $H$ of $\Gamma$ and any group homomorphism $\alpha: H \rightarrow \mathcal{P U}(\mathcal{H})$, we can construct the $\Gamma-$ equivariant principal $\mathcal{P U}(\mathcal{H})$-bundle

$$
\Gamma \times_{\alpha} \mathcal{P U}(\mathcal{H}) \rightarrow \Gamma / H
$$

where $\Gamma \times_{\alpha} \mathcal{P U}(\mathcal{H})$ is the quotient of $\Gamma \times \mathcal{P U}(\mathcal{H})$ under the left $H$-action given by $h \cdot(\gamma, g)=\left(\gamma h^{-1}, \alpha(h) g\right)$. The associated bundle

$$
\left(\Gamma \times_{\alpha} \mathcal{P U}(\mathcal{H})\right) \times_{\mathcal{P U}(\mathcal{H})} \operatorname{Fred}(\mathcal{H}) \cong \Gamma \times_{\alpha} \operatorname{Fred}(\mathcal{H})
$$

is equivalent to the quotient of $\Gamma \times \operatorname{Fred}(\mathcal{H})$ under the left $H$-action which is given by $h \cdot(\gamma, F)=\left(\gamma h^{-1}, \alpha(h) F \alpha(h)^{-1}\right)$. Therefore the space of $\Gamma$-equivariant sections of the bundle

$$
\Gamma \times{ }_{\alpha} \operatorname{Fred}(\mathcal{H}) \rightarrow \Gamma / H
$$

is homeomorphic to the space of $\alpha$-invariant operators

$$
\operatorname{Fred}(\mathcal{H})^{\alpha}:=\left\{F \in \operatorname{Fred}(\mathcal{H}) \mid \alpha(h) F \alpha(h)^{-1}=F \text { for all } h \in H\right\} .
$$

Now, the index map

$$
\text { index: } \operatorname{Fred}(\mathcal{H})^{\alpha} \rightarrow R(\tilde{H}), \quad F \mapsto \operatorname{ker}(F)-\operatorname{coker}(F)
$$

maps an $\alpha$-invariant operator to an element of the Grothendieck ring of representations of $\widetilde{H}:=\alpha^{*} \mathcal{U}(\mathcal{H})$, group which was defined in diagram (15-2).

In order for the homotopy groups of $\operatorname{Fred}(\mathcal{H})^{\alpha}$ to represent the local coefficients for twisted equivariant $K$-theory, we know that we need to impose two conditions on the homomorphism $\alpha: H \rightarrow \mathcal{P U}(\mathcal{H})$ : 
- The image of the index map is a subgroup of $R(\tilde{H})$; this in order for the connected components $\pi_{0}\left(\operatorname{Fred}(\mathcal{H})^{\alpha}\right)$ to be a group.

- All the representations of $\tilde{H}$, on which $S^{1}=\operatorname{ker}(\widetilde{H} \rightarrow H)$ acts by multiplication, must appear on the image of the index map; this in order to obtain a theory compatible with restriction of representations.

If a homomorphism $\alpha$ satisfies these two conditions, then the index map

$$
\text { index: } \pi_{0}\left(\operatorname{Fred}(\mathcal{H})^{\alpha}\right) \stackrel{\cong}{\rightarrow} R_{\alpha}(H),
$$

induces an isomorphism between the path components of $\operatorname{Fred}(\mathcal{H})^{\alpha}$ and the Grothendieck group

$$
R_{\alpha}(H):=\left\{V \in R(\tilde{H}) \mid S^{1}=\operatorname{ker}(\tilde{H} \rightarrow H) \text { acts by multiplication on } V\right\}
$$

of so called $\alpha$-twisted $H$-representations.

Homomorphisms that satisfy these two conditions will be called stable; cf [4, Section 6].

Definition 15.11 (Stable homomorphism) A homomorphism $\alpha: H \rightarrow \mathcal{P U}(\mathcal{H})$ is stable if all irreducible representations of $\widetilde{H}$ on which $S^{1}=\operatorname{ker}(\widetilde{H} \rightarrow H)$ acts by multiplication appear infinitely number of times.

Define the set $\mathcal{S}$ of stable local representations for almost free $\Gamma$-actions by

$$
\mathcal{S}=\{(H, \alpha) \mid H \in \mathcal{F} \mathcal{I} \mathcal{N}(\Gamma) \text { and } \alpha: H \rightarrow \mathcal{P U}(\mathcal{H}) \text { is a stable homomorphism }\} .
$$

Note that $\mathcal{S}$ is indeed a family of local representations since conjugation of stable homomorphisms is also stable and the restriction of a stable homomorphism to a subgroup is stable; this last statement follows from the Frobenius reciprocity theorem since all irreducible $\left.\alpha\right|_{K}$-twisted representations of $K$ subgroup of $H$ may be obtained from the $\alpha$-twisted representations of $H$.

Now let $B$ be an almost free $\Gamma-\mathrm{CW}$-complex. Then a $\Gamma$-equivariant principle $\mathcal{P U}(\mathcal{H})-$ bundle $p: E \rightarrow B$ whose family of local representations is contained in $\mathcal{S}$ is the same as a $\Gamma$-equivariant stable projective unitary bundle over $B$ in the sense of [5, Definition 2.2]. Note that the existence of the local data appearing in [5, Definition 2.2] is automatically satisfied by Theorem 9.1 .

We obtain from Theorem 11.4 a universal $\Gamma$-equivariant stable projective unitary bundle $p: E(\Gamma, \mathcal{P U}(\mathcal{H}), \mathcal{S}) \rightarrow B(\Gamma, \mathcal{P U}(\mathcal{H}), \mathcal{S})$ such that for any almost free $\Gamma-\mathrm{CW}$-complex $X$ the pullback construction yields a bijection from $[X, B(\Gamma, \mathcal{P U}(\mathcal{H}), \mathcal{S})]^{\Gamma}$ to the set of isomorphism classes of $\Gamma$-equivariant stable projective unitary bundles over $X$. This generalizes [5, Theorem 3.4], where $\Gamma$ is assumed to be discrete. Summarizing, we get the following. 
Theorem 15.12 (Universal $\Gamma$-equivariant stable projective bundle) Let $\Gamma$ be a Hausdorff topological group. Then the bundle

$$
\mathcal{P U}(\mathcal{H}) \rightarrow E(\Gamma, \mathcal{P U}(\mathcal{H}), \mathcal{S}) \stackrel{p}{\rightarrow} B(\Gamma, \mathcal{P U}(\mathcal{H}), \mathcal{S})
$$

is a universal $\Gamma$-equivariant stable projective unitary bundle for almost free $\Gamma-C W-$ complexes.

For $X$ a proper $\Gamma-\mathrm{CW}$-complex, the set of the isomorphism classes of projective unitary $\Gamma$-equivariant stable bundles over $X$ has been denoted by $\operatorname{Bun}_{\mathrm{st}}^{\Gamma}(X, \mathcal{P U}(\mathcal{H}))$ in [5, Definition 2.2]; we have then the canonical isomorphisms of sets

$$
\operatorname{Bun}_{\mathrm{st}}^{\Gamma}(X, \mathcal{P U}(\mathcal{H}))=\operatorname{Bundle}_{\Gamma, \mathcal{P U}(\mathcal{H}), \mathcal{S}}(X) \stackrel{\cong}{\rightarrow}[X, B(\Gamma, \mathcal{P U}(\mathcal{H}), \mathcal{S})]^{\Gamma} .
$$

Therefore the $\Gamma$-equivariant stable projective unitary bundle

$$
\mathcal{P U}(\mathcal{H}) \rightarrow E(\Gamma, \mathcal{P U}(\mathcal{H}), \mathcal{S}) \stackrel{p}{\rightarrow} B(\Gamma, \mathcal{P U}(\mathcal{H}), \mathcal{S})
$$

is the universal twist in equivariant $K$-theory for almost free $\Gamma-\mathrm{CW}$-complexes. In particular, for a fixed $\Gamma$-equivariant map $f: X \rightarrow B(\Gamma, \mathcal{P U}(\mathcal{H}), \mathcal{S})$ with $X$ almost free $\Gamma-\mathrm{CW}$-complex, the twisted equivariant $K$-theory groups of the pair $(X, f)$ can be defined as

$$
K_{\Gamma}^{-i}(X, f):=\pi_{i}\left(\operatorname{Sections}\left(f^{*} E(\Gamma, \mathcal{P U}(\mathcal{H}), \mathcal{S}) \times_{\mathcal{P U}(\mathcal{H})} \operatorname{Fred}(\mathcal{H})\right)^{\Gamma}\right),
$$

namely, as the homotopy groups of the space of $\Gamma$-equivariant sections of the associated $\operatorname{Fred}(\mathcal{H})$-bundle; see [5, Appendix A] for further properties of the twisted equivariant $K$-theory groups defined in this way.

Now, in the case that $\Gamma$ is furthermore a Lie group we can show that there is an isomorphism between the set $\operatorname{Bundle}_{\mathrm{st}}^{\Gamma}(X, \mathcal{P U}(\mathcal{H}))$ and $H^{3}\left(E \Gamma \times_{\Gamma} X, \mathbb{Z}\right)$.

Theorem 15.13 Let $\Gamma$ be a Lie group and $X$ be an almost free $\Gamma-C W$ complex. Then the map

$$
\begin{aligned}
\operatorname{map}\left(\operatorname{id}_{X}, \psi\right): \operatorname{map}(X, B(\Gamma, \mathcal{P U}(\mathcal{H}), \mathcal{S})) & \rightarrow \operatorname{map}(X, \operatorname{map}(E \Gamma, B \mathcal{P U}(\mathcal{H}))), \\
f & \mapsto \psi \circ f
\end{aligned}
$$

is a weak $\Gamma$-homotopy equivalence.

In particular we obtain bijections

$$
\begin{aligned}
& \text { Bundle }_{\Gamma, \mathcal{P U}(\mathcal{H}, \mathcal{S}}(X) \cong[X, B(\Gamma, \mathcal{P U}(\mathcal{H}), \mathcal{S})]^{\Gamma} \stackrel{\cong}{\rightarrow}[X, \operatorname{map}(E \Gamma, B \mathcal{P U}(\mathcal{H}))]^{\Gamma}, \\
& {\left[E \Gamma \times_{\Gamma} X, B \mathcal{P U}(\mathcal{H})\right] \cong H^{3}\left(E \Gamma \times_{\Gamma} X, \mathbb{Z}\right) \text {. }}
\end{aligned}
$$


Proof For $H$ a finite group of $\Gamma$ we just need to show that the induced map

$$
\psi^{H}: B(\Gamma, \mathcal{P U}(\mathcal{H}), \mathcal{S})^{H} \rightarrow \operatorname{map}(E \Gamma, B \mathcal{P U}(\mathcal{H}))^{H} \simeq \operatorname{map}(B H, B \mathcal{P U}(\mathcal{H}))
$$

is a weak homotopy equivalence; the rest of the proof is formal and it is equivalent to the one that appears in the proof of Theorem 14.6.

Let $\operatorname{hom}_{\mathcal{S}}(H, \mathcal{P U}(\mathcal{H}))$ be the set of stable homomorphisms. In [5, Proposition 1.5] it is shown that the map

$$
\operatorname{hom}_{\mathcal{S}}(H, \mathcal{P U}(\mathcal{H})) / \mathcal{P U}(\mathcal{H}) \stackrel{\cong}{\rightarrow} \operatorname{Ext}\left(H, S^{1}\right), \quad \alpha \mapsto \tilde{H}=\alpha^{*} \mathcal{U}(\mathcal{H})
$$

is an isomorphism of sets, where $\operatorname{Ext}\left(H, S^{1}\right)$ denotes the set of isomorphism classes of central $S^{1}$-extensions of $H$. Now, we have the isomorphism of sets

$$
\operatorname{Ext}\left(H, S^{1}\right) \cong H^{3}(B H, \mathbb{Z}) \cong[B H, B \mathcal{P U}(\mathcal{H})],
$$

since $\mathcal{P U}(\mathcal{H})$ is $K(\mathbb{Z}, 2)$-space by Kuiper's Theorem [16]. Since by Theorem 13.1 we know that

$$
\operatorname{hom}_{\mathcal{S}}(H, \mathcal{P U}(\mathcal{H})) / \mathcal{P U}(\mathcal{H}) \stackrel{\cong}{\longrightarrow} \pi_{0}\left(B(\Gamma, \mathcal{P U}(\mathcal{H}), \mathcal{S})^{H}\right),
$$

we obtain the desired isomorphism at the level of connected components

$$
\pi_{0}\left(\psi^{H}\right): \pi_{0}\left(B(\Gamma, \mathcal{P U}(\mathcal{H}), \mathcal{S})^{H}\right) \stackrel{\cong}{\rightarrow} \pi_{0}\left(\operatorname{map}(E \Gamma, B \mathcal{P U}(\mathcal{H}))^{H}\right) .
$$

Take now $\alpha \in \operatorname{hom}_{\mathcal{S}}(H, \mathcal{P U}(\mathcal{H}))$. By Theorem 13.1 we have a weak homotopy equivalence

$$
B C_{\mathcal{P U}(\mathcal{H})}(\alpha) \stackrel{\simeq}{\rightarrow} B(\Gamma, \mathcal{P U}(\mathcal{H}), \mathcal{S})_{\alpha}^{H}
$$

by [5, Theorem 1.8] we know that the homotopy groups of $C_{\mathcal{P U}(\mathcal{H})}(\alpha)$ are

$$
\pi_{j}\left(C_{\mathcal{P U}(\mathcal{H})}(\alpha)\right)= \begin{cases}\operatorname{hom}\left(H, S^{1}\right) & \text { for } j=0 \\ \mathbb{Z} & \text { for } j=2 \\ 0 & \text { otherwise }\end{cases}
$$

By a simple calculation the homotopy groups for $j>0$ of $\operatorname{map}(E \Gamma, B \mathcal{P U}(\mathcal{H}))^{H}$ are

$$
\pi_{j}\left(\operatorname{map}(E \Gamma, B \mathcal{P U}(\mathcal{H}))^{H}\right)= \begin{cases}H^{2}(B H, \mathbb{Z})=\operatorname{hom}\left(H, S^{1}\right) & \text { for } j=1, \\ H^{0}(B H, \mathbb{Z})=\mathbb{Z} & \text { for } j=3, \\ 0 & \text { otherwise. }\end{cases}
$$

Thus we see the homotopy groups of $B(\Gamma, \mathcal{P U}(\mathcal{H}), \mathcal{S})^{H}$ and $\operatorname{map}(E \Gamma, B \mathcal{P U}(\mathcal{H}))^{H}$ are isomorphic and concentrated in degrees $j=0$ and $j=2$. 
Now, in order to show that indeed $\psi^{H}$ induces the desired isomorphism on homotopy groups we need to resort to the proof of Theorem 13.1. For $E=E(\Gamma, \mathcal{P U}(\mathcal{H}), \mathcal{S})$ we know that

$$
\left.E\right|_{B(\Gamma, \mathcal{P U}(\mathcal{H}), \mathcal{S})_{\alpha}^{H}} \cong \mathcal{P U}(\mathcal{H}) \times_{C_{\mathcal{P U}(\mathcal{H})}(\alpha)} E^{K(H, \alpha)}
$$

with $E^{K(H, \alpha)} \rightarrow B(\Gamma, \mathcal{P U}(\mathcal{H}), \mathcal{S})_{\alpha}^{H}$ a principal $C_{\mathcal{P U}(\mathcal{H})}(\alpha)$-bundle. Therefore the map that defines $\psi^{H}$,

$$
E \Gamma / H \times B(\Gamma, \mathcal{P U}(\mathcal{H}), \mathcal{S})_{\alpha}^{H} \rightarrow B \mathcal{P U}(\mathcal{H}),
$$

is weakly homotopically equivalent to the map obtained by applying the classifying space functor

$$
B H \times B C_{\mathcal{P U}(\mathcal{H})}(\alpha) \rightarrow B \mathcal{P U}(\mathcal{H})
$$

to the group homomorphism

$$
\phi: H \times C_{\mathcal{P U}(\mathcal{H})}(\alpha) \rightarrow \mathcal{P U}(\mathcal{H}), \quad(h, g) \mapsto \alpha(h) g .
$$

Since all the $\tilde{H}$ representations defined by $\alpha$ appear infinitely number of times, then it follows that the inclusion of groups $C_{\mathcal{P U}(\mathcal{H})}(\alpha) \subset \mathcal{P U}(\mathcal{H})$ induces an isomorphism on the second homotopy groups $\pi_{2}$; this implies that $\psi^{H}$ induces an isomorphism on the third homotopy groups $\pi_{3}$. It remains to show that $\psi^{H}$ induces an isomorphism at the level of fundamental groups.

The map $\phi$ permits us to define a homeomorphism

$$
C_{\mathcal{P U}(\mathcal{H})}(\alpha) \rightarrow \operatorname{hom}_{\alpha}(\mathbb{Z} \times H, \mathcal{P U}(\mathcal{H})), \quad g \mapsto\left[(n, h) \mapsto g^{n} \alpha(h)\right],
$$

where

$$
\operatorname{hom}_{\alpha}(\mathbb{Z} \times H, \mathcal{P U}(\mathcal{H})):=\left\{\beta \in \operatorname{hom}_{\alpha}(\mathbb{Z} \times H, \mathcal{P U}(\mathcal{H})) \mid \beta(0, h)=\alpha(h)\right\}
$$

Moreover we can define a group homomorphism (see [5, Lemma 1.6])

$$
C_{\mathcal{P U}(\mathcal{H})}(\alpha) \rightarrow \operatorname{hom}\left(H, S^{1}\right), \quad g \mapsto\left(h \mapsto \widetilde{\alpha}(\tilde{h}) \circ \bar{g} \circ \widetilde{\alpha}(\tilde{h})^{-1} \circ \bar{g}^{-1}\right),
$$

where $\tilde{h}$ is any lift of $h$ in $\tilde{H}$ and $\bar{g}$ is any lift of $g$ on $\mathcal{U}(\mathcal{H})$, such that it becomes an isomorphism of groups on path connected components

$$
\pi_{0}\left(C_{\mathcal{P U}(\mathcal{H})}(\alpha)\right) \stackrel{\cong}{\rightrightarrows} \operatorname{hom}\left(H, S^{1}\right) .
$$


Therefore we obtain the following commutative diagram:

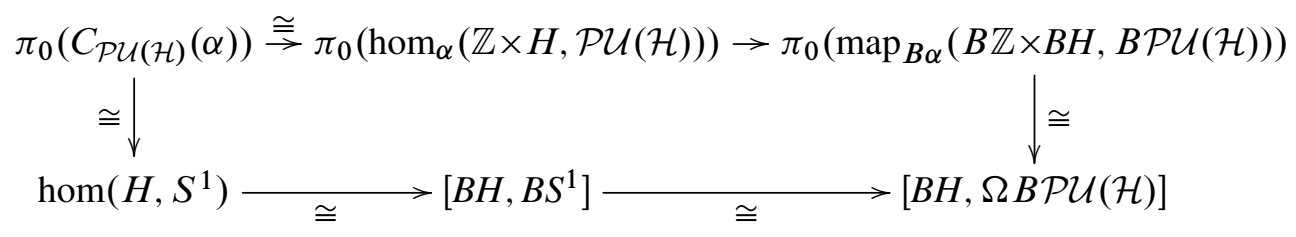

where

$$
\operatorname{map}_{B \alpha}(B \mathbb{Z} \times B H, B \mathcal{P U}(\mathcal{H})):=\{f: B \mathbb{Z} \times B H \rightarrow B \mathcal{P U}(\mathcal{H}) \mid f(*, y)=B \alpha(y)\},
$$

the right vertical arrow is given by the adjoint map since $B \mathbb{Z} \simeq S^{1}$ and $\Omega$ denotes the based loop space functor, and the bottom right map is defined via the homotopy equivalence $B S^{1} \rightarrow \Omega B \mathcal{P U}(\mathcal{H})$, which induces the homotopy equivalence $B S^{1} \rightarrow \mathcal{P U}(\mathcal{H})$. Hence we conclude that at the level of fundamental groups the map $\phi$ induces an isomorphism

$$
\pi_{1}\left(B C_{\mathcal{P U}(\mathcal{H})}(\alpha)\right) \stackrel{\cong}{\rightarrow} \pi_{1}(\operatorname{map}(B H, B \mathcal{P U}(\mathcal{H})))
$$

and thus $\psi^{H}$ induces the desired isomorphism at the level of fundamental groups.

\section{Appendix A: Compactly generated spaces}

We briefly recall some basics about compactly generated spaces. More information and proofs can be found in [33]. A topological space $X$ is compactly generated if it is a Hausdorff space and a set $A \subseteq X$ is closed if and only if for any compact subset $C \subset X$ the intersection $C \cap A$ is a closed subspace of $C$.

Every locally compact space, and every space satisfying the first axiom of countability, eg a metrizable space, is compactly generated. If $p: X \rightarrow Y$ is an identification of topological spaces and $X$ is compactly generated and $Y$ is Hausdorff, then $Y$ is compactly generated. A closed subset of a compactly generated space is again compactly generated. For open subsets one has to be careful as it is explained in Section A.1.

\section{A.1 Open subsets}

Recall that a topological space $X$ is called regular if for any point $x \in X$ and closed set $A \subseteq X$ there exists open subsets $U$ and $V$ with $x \in U, A \subseteq V$ and $U \cap V=\varnothing$. A space is called locally compact if every $x \in X$ possesses a compact neighborhood. Equivalently, for every $x \in X$ and open neighborhood $U$ there exist an open neighborhood $V$ of $x$ such that the closure of $V$ in $X$ is compact and contained in $U$; see 
Munkres [27, Lemma 8.2 in Section 3-8 on page 185]. Recall the Definition 1.1 saying that an open subset $U \subseteq X$ is called quasiregular if for any $x \in U$ there exists an open neighborhood $V_{x}$ whose closure in $X$ is contained in $U$.

Lemma A.1 (i) Let $B$ be a (compactly generated) space. A quasiregular open subset $U \subseteq B$ equipped with the subspace topology is compactly generated.

(ii) Let $f: X \rightarrow Y$ be a (continuous) map between (not necessarily compactly generated) spaces. If $V \subseteq Y$ is a quasiregular open subset, then $f^{-1}(V) \subseteq X$ is a quasiregular open subset.

(iii) The intersection of finitely many quasiregular open subsets is again a quasiregular open subset.

(iv) A space is regular if and only if every open subset is quasiregular.

(v) Any locally compact space, any metrizable space and every $C W$-complex are regular.

(vi) Every $\Gamma$-invariant open subset of a $\Gamma-C W$-complex is quasiregular and compactly generated when equipped with the subspace topology.

Proof (i) See [33, page 135].

(ii) Consider a point $x \in f^{-1}(V)$. Choose an open set $W$ of $Y$ such that $f(x) \in W$ and the closure of $W$ in $B$ is contained in $V$. Then $f^{-1}(W)$ is an open subset of $X$ which contains $x$ and whose closure in $X$ is contained in $f^{-1}(V)$.

(iii) Let $U_{1}, U_{2}, \ldots, U_{r}$ be quasiregular open subsets. Consider $x \in U:=\bigcap_{i=1}^{r} U_{i}$. Choose for every $i=1,2 \ldots, r$ an open subset $V_{i}$ with $x \in V_{i}$ such that the closure $\bar{V}_{i}$ of $V_{i}$ in $B$ is contained in $U_{i}$. Put $V:=\bigcap_{i=1}^{r} V_{i}$. Then $x \in V$ and $\bar{V} \subseteq \bigcap_{i=1}^{r} \bar{V}_{i} \subseteq U$. Hence $U$ is a quasiregular open subset.

(iv) See [27, Lemma 2.1 in Section 4-2 on page 196].

(v) This is obvious for locally compact spaces. Metrizable spaces are treated by Munkres in [27, Theorem 2.3, Section 4-2, page 198]. Every CW-complex is paracompact (see [25]) and hence in particular regular; see [27, Theorem 4.1, Section 6-4, page 255].

(vi) The projection $X \rightarrow X / \Gamma$ is open and $X / \Gamma$ is a CW-complex by Lemma A.7. Now apply assertions (i), (iv) and (v). 


\section{A.2 The retraction functor $k$}

There is a construction which assigns to a topological Hausdorff space $X$ a new topological space $k(X)$ such that $X$ and $k(X)$ have the same underlying sets, $k(X)$ is compactly generated, $X$ and $k(X)$ have the same compact subsets, the identity $k(X) \rightarrow X$ is continuous and is a homeomorphism if and only if $X$ is compactly generated. Namely, define the new topology on $k(X)$ by declaring a subset $A \subseteq X$ to be closed if and only if for every compact subset of $X$ the intersection $A \cap C$ is a closed subset of $C$.

\section{A.3 Mapping spaces, product spaces and subspaces}

Given two compactly generated spaces $X$ and $Y$, denote by $\operatorname{map}(X, Y)_{\text {k.o. the set of }}$ maps $X \rightarrow Y$ with the compact-open topology, ie a subbasis for the compact-open topology is given by the sets $W(C, U)=\{f: X \rightarrow Y \mid f(C) \subseteq U\}$, where $C$ runs through the compact subsets of $X$ and $U$ runs though the open subsets of $Y$. Notice

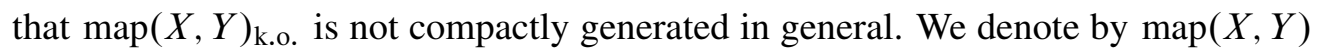
the topological space given by $k\left(\operatorname{map}(X, Y)_{\text {k.o. }}\right)$.

If $X$ and $Y$ are compactly generated spaces, then $X \times Y$ stands for $k\left(X \times_{p} X\right)$, where $X \times{ }_{p} Y$ is the topological space with respect to the "classical" product topology.

If $A \subseteq X$ is a subset of a compactly generated space, the subspace topology means that we take $k\left(A_{\mathrm{st}}\right)$ for $A_{\text {st }}$ the topology space given by the "classical" subspace topology on $A$.

Roughly speaking, all the usual constructions of topologies are made compactly generated by passing from $Y$ to $k(Y)$ in order to stay within the category of compactly generated spaces.

\section{A.4 Basic features of the category of compactly generated spaces}

The category of compactly generated spaces has the following convenient features.

- A map $f: X \rightarrow Y$ of compactly generated spaces is continuous if and only if its restriction $\left.f\right|_{C}: C \rightarrow Y$ to any compact subset $C \subseteq X$ is continuous.

- The product of two identifications is again an identification.

- If $X$ is locally compact and $Y$ compactly generated, then $X \times Y$ and $X \times_{p} Y$ are the same topological spaces.

- The product of a $\Gamma_{1}-\mathrm{CW}-$ complex and of a $\Gamma_{2}-\mathrm{CW}-$ complex is a $\Gamma_{1} \times \Gamma_{2}-$ CW-complex. 
- If $X, Y$, and $Z$ are compactly generated spaces, then the obvious maps

$$
\begin{aligned}
& \operatorname{map}(X, \operatorname{map}(Y, Z)) \stackrel{\cong}{\rightarrow} \operatorname{map}(X \times Y, Z), \\
& \operatorname{map}(X, Y \times Z) \stackrel{\cong}{\rightarrow} \operatorname{map}(X, Y) \times \operatorname{map}(X, Z)
\end{aligned}
$$

are homeomorphisms and the map given by composition

$$
\operatorname{map}(X, Y) \times \operatorname{map}(Y ; Z) \rightarrow \operatorname{map}(X, Z)
$$

is continuous.

- Given a pushout in the category of compactly generated spaces, its product with a compactly generated space is again a pushout in the category of compactly generated spaces.

\section{A.5 Space of homomorphisms}

Let $H$ and $G$ be (compactly generated) topological groups. Let $\operatorname{hom}(H, G)$ be the set of group homomorphisms from $H$ to $G$. It is obviously a subset of $\operatorname{map}(H, G)$. The proof of the next result is a typical formal proof using the convenient basic properties of the category of compactly generated spaces.

Lemma A.2 The subset $\operatorname{hom}(H, G)$ of $\operatorname{map}(H, G)$ is closed.

Proof Consider the map

$$
u: H \times H \times \operatorname{map}(H, G) \rightarrow G, \quad\left(h_{1}, h_{2}, \alpha\right) \mapsto \alpha\left(h_{1}\right) \cdot \alpha\left(h_{2}\right) \cdot \alpha\left(h_{1} h_{2}\right)^{-1} .
$$

It is continuous since it can be written as a composition of maps each of which is obviously continuous by the basic features presented in Section A.4:

$$
\begin{aligned}
H \times H \times \operatorname{map}(H, G) \stackrel{u_{1}}{\longrightarrow} H \times \operatorname{map}(H, G) \times H \times \operatorname{map}(H, G) \times H \times \operatorname{map}(H, G) \\
\stackrel{u_{2}}{\longrightarrow} G \times G \times G \stackrel{u_{3}}{\longrightarrow} G,
\end{aligned}
$$

where

$$
\begin{aligned}
u_{1}\left(h_{1}, h_{1}, \alpha\right) & =\left(h_{1}, \alpha, h_{2}, \alpha, h_{1} h_{2}, \alpha\right), \\
u_{2}\left(h_{1}, \alpha_{1}, h_{2}, \alpha_{2}, h_{3}, \alpha_{3}\right) & =\left(\alpha_{1}\left(h_{1}\right), \alpha_{2}\left(h_{2}\right), \alpha_{3}\left(h_{3}\right)\right), \\
\alpha_{3}\left(g_{1}, g_{2}, g_{3}\right) & =g_{1} g_{2} g_{3}^{-1} .
\end{aligned}
$$

Its adjoint is the continuous map

$$
v: \operatorname{map}(H, G) \rightarrow \operatorname{map}(H \times H, G), \quad \alpha \mapsto v(\alpha)
$$


given by $v(\alpha)\left(h_{1}, h_{2}\right):=\alpha\left(h_{1}\right) \cdot \alpha\left(h_{2}\right) \cdot \alpha\left(h_{1} h_{2}\right)^{-1}$. The evaluation map

$$
\text { ev: } \operatorname{map}(H, G) \rightarrow G, \quad \alpha \mapsto \alpha(1)
$$

is continuous by Section A.4. Since $\operatorname{hom}(H, G)$ is the intersection of $v^{-1}(\{1\})$ and $\mathrm{ev}^{-1}(\{1\})$ and $\{1\} \subseteq G$ is closed, $\operatorname{hom}(H, G)$ is a closed subset of $\operatorname{map}(H, G)$.

We will equip $\operatorname{hom}(H ; G)$ with the subspace topology coming from $\operatorname{map}(H, G)$. Notice that it is automatically compactly generated so that $\operatorname{hom}(H ; G)$ agrees with $k(\operatorname{hom}(H ; G))$.

We leave the formal proof to the reader that the conjugation action

$$
G \times \operatorname{hom}(H, G) \rightarrow \operatorname{hom}(H, G), \quad(g, \alpha) \mapsto c_{g} \circ \alpha
$$

is continuous, where $c_{g}: G \rightarrow G$ sends $g^{\prime}$ to $g g^{\prime} g^{-1}$.

We also include the following results.

Lemma A.3 Let $p: X \rightarrow Y$ be an identification and $Z$ be a space. Then the induced map

$$
p^{*}: \operatorname{map}(Y, Z) \rightarrow \operatorname{map}(X, Z), \quad f \mapsto f \circ p,
$$

is a closed embedding, ie has closed image and the induced map $q: \operatorname{map}(Y, Z) \rightarrow \operatorname{im}\left(p^{*}\right)$ is a homeomorphism.

Proof Obviously $p^{*}$ is injective and continuous.

Next we show that the image of $p^{*}$ is closed. Consider $f: X \rightarrow Z$ which is not in the image of $p^{*}$. Then there exists $x_{0}$ and $x_{1}$ in $X$ with $f\left(x_{0}\right) \neq f\left(x_{1}\right)$ and $p\left(x_{0}\right)=p\left(x_{1}\right)$. Since the map

$$
\text { ev: } \operatorname{map}(X, Z) \mapsto Z \times Z, \quad f \mapsto\left(f\left(x_{0}\right), f\left(x_{1}\right)\right)
$$

is continuous, the preimage of the open set $\left\{\left(z_{0}, z_{1}\right) \in Z \times Z \mid z_{0} \neq z_{1}\right\}$ under ev is an open set which contains $f$ and does not meet $\operatorname{im}\left(p^{*}\right)$. Hence $\operatorname{im}\left(p^{*}\right)$ is closed in $\operatorname{map}(X, Z)$.

It remains to show that the inverse of $q$

$$
q^{-1}: \operatorname{im}\left(p^{*}\right) \rightarrow \operatorname{map}(Y, Z)
$$

is continuous. Consider an open subset $V \subset \operatorname{map}(Y, Z)_{\text {k.o. }}$. By the definition of the compact open topology we can find an index set $I$, for every element $i \in I$ a finite 
index set $J_{i}$, and for every $i \in I$ and $j_{i} \in J_{i}$ an open subset $U_{i, j_{i}} \subseteq Y$ and a compact subset $C_{i, j_{i}} \subseteq Z$ such that

$$
V=\bigcup \bigcap_{i \in I} W\left(U_{i, j_{i}}, C_{i, j_{i}}\right),
$$

where $W\left(U_{i, j_{i}}, C_{i, j_{i}}\right)=\left\{f \in \operatorname{map}(Y, Z) \mid f\left(U_{i, j_{i}}\right) \subseteq C_{i, j_{i}}\right\}$. We have

$$
\begin{aligned}
q(V)=p^{*}\left(\bigcup_{i \in I} \bigcap_{j_{i} \in J_{i}} W\left(U_{i, j_{i}}, C_{i, j_{i}}\right)\right) & =\bigcup \bigcap_{i \in I} p^{*}\left(W\left(U_{i, j_{i}}, C_{i, j_{i}}\right)\right) \\
& =\bigcup \bigcap_{i \in I}\left(W\left(p^{-1}\left(U_{i, j_{i}}\right), C_{i, j_{i}}\right) \cap \operatorname{im}\left(p^{*}\right)\right) \\
& =\left(\bigcup_{i \in I} \bigcap_{j_{i} \in J_{i}} W\left(p^{-1}\left(U_{i, j_{i}}\right), C_{i, j_{i}}\right)\right) \cap \operatorname{im}\left(p^{*}\right),
\end{aligned}
$$

where $W\left(p^{-1}\left(U_{i, j_{i}}\right), C_{i, j_{i}}\right)=\left\{f \in \operatorname{map}(X, Z) \mid f\left(p^{-1}\left(U_{i, j_{i}}\right)\right) \subseteq C_{i, j_{i}}\right\}$. Since $\bigcup_{i \in I} \bigcap_{j_{i} \in J_{i}} W\left(p^{-1}\left(U_{i, j_{i}}\right), C_{i, j_{i}}\right)$ is an open subset of $\operatorname{map}(Y, Z)_{\mathrm{k} . o \text {. }}$, the subset $q(V)$

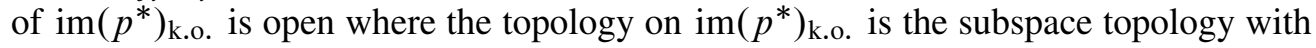
respect to the inclusion $\operatorname{im}\left(p^{*}\right) \rightarrow \operatorname{map}(X, Z)_{\mathrm{k} . \mathrm{o} .}$. Therefore we have that the map $q_{\mathrm{k} . \mathrm{o}}^{-1}: \operatorname{im}\left(p^{*}\right)_{\mathrm{k} . \mathrm{o} .} \rightarrow \operatorname{map}(Y, Z)_{\mathrm{k} . o .}$ is continuous. This implies that the map

$$
k\left(q_{\text {k.o. }}^{-1}\right): k\left(\operatorname{im}\left(p^{*}\right)_{\text {k.o. }}\right) \rightarrow k\left(\operatorname{map}(Y, Z)_{\text {k.o. }}\right)=\operatorname{map}(Y, Z)
$$

is continuous. The identity induces a continuous map

$$
i_{0}: \operatorname{map}(X, Z)=k\left(\operatorname{map}(X, Z)_{\mathrm{k} . \mathrm{o} .}\right) \rightarrow \operatorname{map}(X, Z)_{\mathrm{k} . \mathrm{o} .}
$$

and hence by restriction a continuous map $i_{1}: \operatorname{im}\left(p^{*}\right) \rightarrow \operatorname{im}\left(p^{*}\right)_{\mathrm{k} . o .}$. If we apply $k$ to it and use the fact that $\operatorname{im}\left(p^{*}\right)$ is compactly generated, we obtain a continuous map

$$
i_{1}: \operatorname{im}\left(p^{*}\right) \rightarrow k\left(\operatorname{im}\left(p^{*}\right)_{\mathrm{k} . o .}\right) .
$$

Since $q^{-1}: \operatorname{im}\left(p^{*}\right) \rightarrow \operatorname{map}(Y, Z)$ is the composite of the two continuous maps $k\left(q_{\mathrm{k} . o .}^{-1}\right)$ and $i_{1}$ above, it is continuous itself. This finishes the proof of Lemma A.3.

Lemma A.4 For every group $G$ and $G$-spaces $Y$ and $Z$ the map

$$
Q_{Y, Z}: \operatorname{map}_{G}(Y, Z) \rightarrow \operatorname{map}_{G}(Y / G, Z / G), \quad f \mapsto f / G,
$$

is continuous.

Proof Let $p_{Y}: Y \rightarrow Y / G$ and $p_{Z}: Z \rightarrow Z / G$ be the projections. The map

$$
\left(p_{Z}\right)_{*}: \operatorname{map}(Y, Z) \rightarrow \operatorname{map}(Y, Z / G), \quad f \mapsto p_{Z} \circ f
$$


is continuous and hence induces a continuous map

$$
p_{Z}: \operatorname{map}_{G}(Y, Z) \rightarrow \operatorname{map}_{G}(Y, Z / G), \quad f \mapsto p_{Z} \circ f .
$$

Therefore the claim follows if we can prove that the map

$$
Q_{Y, Z / G}: \operatorname{map}_{G}(Y, Z / G) \rightarrow \operatorname{map}(Y / G, Z / G), \quad f \mapsto f / G
$$

is continuous. Its inverse is the map

$$
p_{Y}^{*}: \operatorname{map}(Y / G, Z / G) \rightarrow \operatorname{map}_{G}(Y, Z / G), \quad f \mapsto f \circ p_{Y},
$$

which is a homeomorphism by Lemma A.3. This finishes the proof of Lemma A.4.

\section{A.6 Further features of the category of compactly generated spaces}

Lemma A.5 Consider a commutative square of $\Gamma$-spaces:

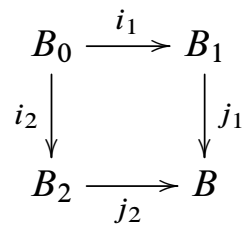

Then:

(i) The square is a $\Gamma$-pushout if and only if it is pushout after forgetting the group action.

(ii) If the square above is a $\Gamma$-pushout and $f: E \rightarrow B$ is a $\Gamma$-map, then the square obtained by the pullback construction

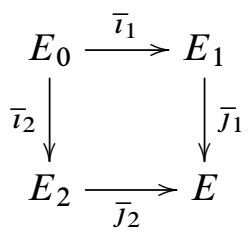

is a $\Gamma$-pushout.

(iii) If the given square is a $\Gamma$-pushout, the square

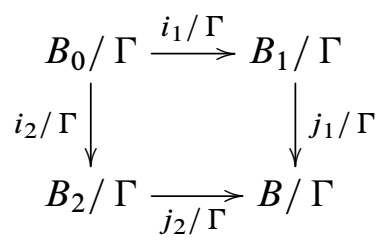

is a pushout of spaces. 
Proof (i) Suppose that the square is a (nonequivariant) pushout. We want to show that it is a $\Gamma$-pushout. Consider $\Gamma$-maps $f_{k}: B_{k} \rightarrow Y$ for $k=0,1,2$ satisfying $f_{k} \circ i_{k}=f_{0}$ for $k=1,2$. Then there is precisely one map $f: B \rightarrow X$ with $f \circ j_{k}=f_{k}$ for $k=1,2$. It remains to show that $f$ is $\Gamma$-equivariant. This follows from the fact that the product of $\Gamma$ with the square above is again a (nonequivariant) pushout. This follows formally from the adjunctions of mapping spaces and the universal property of pushouts.

Now suppose that the square is a $\Gamma$-pushout. Then it is a pushout after forgetting the group action, since there is a bijection

$$
a: \operatorname{map}(B, X) \stackrel{\cong}{\rightarrow} \operatorname{map}_{\Gamma}(B, \operatorname{map}(\Gamma, X)), \quad f \mapsto a(f),
$$

where $a(f)$ sends $b \in B$ to the map $\Gamma \rightarrow X, \gamma \mapsto f(\gamma \cdot b), X$ is a (nonequivariant) space and $\operatorname{map}(\Gamma, X)$ becomes a $\Gamma$-space $\gamma \cdot f(h):=f(h \gamma)$.

(ii) Because of assertion (i), we can assume without loss of generality that $\Gamma$ is trivial. The map $j_{1} \amalg j_{2}: B_{1} \amalg B_{2} \rightarrow B$ is an identification since the given square is a pushout. Then $\operatorname{id}_{E} \times j_{1} \amalg \mathrm{id}_{E} \times j_{2}: E \times B_{1} \amalg E \times B_{2} \rightarrow E \times B$ is an identification; see [33, Theorem 4.4]. Consider $E$ as a closed subspace of $E \times B$ by identifying $e$ with $(e, f(e))$. Then the restriction of $\operatorname{id}_{E} \times j_{1} \amalg \operatorname{id}_{E} \times j_{2}$ to the preimage of $E$ is again an identification which can be identified with $\bar{J}_{1} \amalg \bar{J}_{2}: E_{1} \amalg E_{2} \rightarrow E$. Obviously the square obtained by the pullback construction is a pushout of sets. Hence it is a pushout of spaces.

(iii) This follows from the universal properties of a pushout and the projection maps $B \rightarrow B / \Gamma$.

Lemma A.6 Let $B_{0} \subseteq B_{1} \subseteq B_{2} \subseteq \cdots \subseteq B$ be a filtration of the $\Gamma$-space $B$ by closed $\Gamma$-invariant subspaces.

(i) We have $B=\operatorname{colim}_{n \rightarrow \infty} B_{n}$ in the category of $\Gamma$-spaces if and only if we have after forgetting the $\Gamma$-actions $B=\operatorname{colim}_{n \rightarrow \infty} B_{n}$ in the category of spaces.

(ii) Suppose that we have $B=\operatorname{colim}_{n \rightarrow \infty} B_{n}$ in the category of $\Gamma-$ spaces. Let $f: E \rightarrow B$ be a $\Gamma$-map. Then we obtain a filtration

$$
E_{0} \subseteq E_{1} \subseteq E_{2} \subseteq \cdots \subseteq E
$$

of $E$ by closed $\Gamma$-invariant subspaces $E_{n}=f^{-1}\left(B_{n}\right)$ with the property that $E=\operatorname{colim}_{n \rightarrow \infty} E_{n}$ holds in the category of $\Gamma$-spaces.

(iii) Suppose that $B=\operatorname{colim}_{n \rightarrow \infty} B_{n}$ holds in the category of $\Gamma$-spaces. Then

$$
B_{0} / \Gamma \subseteq B_{1} / \Gamma \subseteq B_{2} / \Gamma \subseteq \cdots \subseteq B / \Gamma
$$

is a filtration of the space $B \Gamma$ by closed subspaces with the property that $B / \Gamma=\operatorname{colim}_{n \rightarrow \infty} B_{n} / \Gamma$ holds in the category of spaces. 
Proof (i) The proof is similar to the one of Lemma A.5(i), using the fact that after forgetting the group actions we obtain a filtration by closed subspaces $\Gamma \times B_{0} \subseteq$ $\Gamma \times B_{1} \subseteq \Gamma \times B_{2} \subseteq \cdots \subseteq \Gamma \times B$ such that $\Gamma \times B=\operatorname{colim}_{n \rightarrow \infty} \Gamma \times B_{n}$ holds in the category of $\Gamma$-spaces; see [33, Theorem 10.3].

(ii) Let $A \subseteq E$ be a subset. Suppose that $A \cap E_{n}$ is closed. We have to show that $A \subseteq E$ is closed, or, equivalently, that for every compact subset $C \subset E$ the space $C \cap A$ is a closed subspace of $C$. Since $f(C)$ is compact, there exists a natural number $n$ with $f(C) \subseteq B_{n}$. This implies $C \subseteq E_{n}$; see [33, Lemma 9.3]. Now the claim follows from $A \cap C=A \cap\left(C \cap E_{n}\right)=\left(A \cap E_{n}\right) \cap C$ since $A \cap E_{n}$ is closed in $E_{n}$ and hence $\left(A \cap E_{n}\right) \cap C$ is closed in $C$.

(iii) This follows from the universal properties of $\operatorname{colim}_{n \rightarrow \infty}$ and of the quotient maps $B_{k} \rightarrow B_{k} / \Gamma$ and $B \rightarrow B / \Gamma_{k}$.

Lemma A.7 If $X$ is a $G-C W$-complex. Let $N \subseteq G$ a normal subgroup and $Q$ be the topological group $G / N$ and pr: $G \rightarrow Q$ be the projection. Suppose for any $x \in X$ that $\operatorname{pr}\left(G_{X}\right)$ is closed in $Q$. (This assumption is automatically satisfied if $N$ is compact or $Q$ is trivial.)

Then $X / N$ is a $Q-C W-$ complex.

Proof Let pr: $G \rightarrow Q$ be the projection. Consider a subgroup $H \subseteq G$ such that $\operatorname{pr}(H)$ is closed in $Q$. Then we obtain a $Q$-homeomorphism $N \backslash(G / H) \stackrel{\cong}{\rightrightarrows} Q / \operatorname{pr}(H)$. Now the claim follows from Lemma A.5(i) and (iii) and Lemma A.6(i) and (iii).

\section{Appendix B: Some properties of locally compact groups}

Let $H$ and $G$ be topological groups. For $\alpha$ in $\operatorname{hom}(H, G)$, let $C_{G}(\alpha)$ be the centralizer of $\alpha$, ie, $C_{G}(\alpha)=\left\{g \in G \mid c_{g} \circ \alpha=\alpha\right\}$, and denote by $G \cdot \alpha$ the orbit of $\alpha$ under the $G$-action. Then the map

$$
\iota_{\alpha}: G / C_{G}(\alpha) \rightarrow G \cdot \alpha, \quad g C_{G}(\alpha) \mapsto c_{g} \circ \alpha
$$

is bijective, continuous and $G$-equivariant. The next two theorems are the main results of this section.

Theorem B.1 Let $H$ be a compact group, let $G$ a locally compact second countable group and $\alpha \in \operatorname{hom}(H, G)$.

Then the connected component $\mathcal{C}_{\alpha}$ of $\alpha$ in $\operatorname{hom}(H, G)$ is contained in the orbit of $\alpha$ under $G$, ie $\mathcal{C}_{\alpha} \subset G \cdot \alpha$. 
Theorem B.2 Let $G$ be a second countable, locally compact group which is almost connected. Let $H$ be a compact group.

Then $\iota_{\alpha}: G / C_{G}(\alpha) \rightarrow G \cdot \alpha$ is a $G$-homeomorphism.

Their respective proofs need some preparation. For a compact space $X$ and a complete metric space $(Y, d)$ we can equip the set of continuous maps from $X$ to $Y$ with the supremum metric

$$
d_{\text {sup }}(\alpha, \beta):=\sup \{d(\alpha(x), \beta(x)) \mid x \in X\},
$$

and obtain a complete metric space; see [27, Theorem 1.4, Section 7-1, page 267]. Moreover, the topology induced by the supremum metric agrees with the compact-open topology by [27, Theorem 4.6, Section 7-4, page 283 and Theorem 5.1, Section 7.5, page 286]. In particular $\operatorname{map}(X, Y)=\operatorname{map}(X, Y)_{\text {k.o. }}$.

The following result is due to Birkhoff [6] and Kakutani [14].

Theorem B.3 A Hausdorff topological group is metrizable if and only if it is first countable. In this case the metric can be taken to be left invariant.

Theorem B.3 implies that for a locally compact first countable group $G$ we can choose a left invariant metric $d_{G}$ inducing the given topology such that $G$ with this metric is complete. Note that completeness follows from local compactness. Namely, we then can find an $\epsilon>0$ such that the closed ball $\bar{B}_{\epsilon}(1)$ around 1 is compact and hence the closed ball $\bar{B}_{\epsilon}(g)$ around any $g \in G$ is compact which implies that every Cauchy sequence contains a subsequence contained in $B_{\epsilon}(g)$ for some $g \in G$ and hence contains a convergent subsequence, by [27, Theorem 7.4, Section 3-7, page 181], and finally we can apply [27, Lemma 1.1, Section 7-1, page 264]

Recall from Section A.5 that we have equipped hom $(H, G)$ with the subspace topology of map $(H, G)$, that $\operatorname{hom}(H, G) \subseteq \operatorname{map}(H, G)$ is closed and that the conjugation action

$$
G \times \operatorname{hom}(H, G) \rightarrow \operatorname{hom}(H, G), \quad(g, \alpha) \mapsto c_{g} \circ \alpha
$$

is continuous. Hence this topology on $\operatorname{hom}(H, G)$ agrees with the topology coming from the compact-open topology on $\operatorname{map}(H, G)$ restricted to $\operatorname{hom}(H ; G)$ as well with the topology associated to the supremum metric restricted to $\operatorname{hom}(H, G)$, and $\operatorname{hom}(H, G)$ is a complete metric space with the supremum metric.

The next theorem is taken from Lee and $\mathrm{Wu}[21$, Theorem I] and obviously implies Theorem B.1. 
Theorem B.4 Let $H$ be a compact group and $G$ a locally compact second countable group. Let $\mathcal{C} \subset \operatorname{hom}(H, G)$ be a connected component of the space $\operatorname{hom}(H, G)$. Then if $\theta$ and $\phi$ are in $\mathcal{C}$, then there exists $g \in G$ with $\theta=c_{g} \circ \phi$.

The following result is taken from Lee and $\mathrm{Wu}$ [21, Theorem II]. Notice that the condition of being almost connected is necessary; see [21, Example, page 412].

Theorem B.5 Let $L$ be a locally compact group which is almost connected. Let $F$ be a compact subgroup of $L$ and denote by $i: F \rightarrow L$ the inclusion. If $\left\{x_{\lambda} \mid \lambda \in \Lambda\right\}$ is a net in $L$ such that the homomorphisms $c_{x_{\lambda}} \circ i$ converge to an element $\theta \in \operatorname{hom}(F, L)$, then there exists an element $y \in L$ such that $\theta=c_{y} \circ i$.

Theorem B.6 Let $G$ be a locally compact group which is almost connected. Let $H$ be a compact group and $\alpha \in \operatorname{hom}(H, G)$. Then the orbit $G \cdot \alpha$ of $\alpha$ under the conjugation action of $G$ is closed in $\operatorname{hom}(H, G)$.

Proof Suppose we have a net $\left\{g_{\lambda} \in G \mid \lambda \in \Lambda\right\}$ of elements of $G$ such that the homomorphisms $c_{g_{\lambda}} \circ \alpha$ converge to an element $\phi \in \operatorname{hom}(H, G)$. We want to show that $\phi$ belongs to $G \cdot \alpha$.

Let us take $L=H \times G$ and $K(H, \alpha):=\{(h, \alpha(h) \mid h \in H\}$ the associated compact subgroup of $H \times G$ defined by $\alpha$. We have then that $L$ is locally compact and $L$ is almost connected. Denote by $i: K(H, \alpha) \rightarrow H \times G$ the inclusion and take the net $\left\{\left(1, g_{\lambda}\right) \in H \times G \mid \lambda \in \Lambda\right\}$ of elements in $H \times G$ induced by the elements $g_{\lambda} \in G$. Note that since the homomorphisms $c_{g_{\lambda}} \circ \alpha$ converge to $\phi \in \operatorname{hom}(H, G)$, the homomorphisms

$$
c_{\left(1, g_{\lambda}\right)} \circ i \in \operatorname{hom}(K(H, \alpha), H \times G)
$$

converge to the homomorphism $\theta_{\phi} \in \operatorname{hom}(K(H, \alpha), H \times G)$ defined by

$$
\theta_{\phi}(h, \alpha(h)):=(h, \phi(h)) .
$$

Applying Theorem B.5 we know that there exists a pair $(y, g) \in H \times G$ such that $\theta_{\phi}=c_{(y, g)} \circ i$; this implies that for all $h \in H$,

$$
(h, \phi(h))=\left(y h y^{-1}, g \alpha(h) g^{-1}\right),
$$

and therefore we can conclude that $\phi=c_{g} \circ \alpha$. Hence $\phi$ belongs to the orbit $G \cdot \alpha$.

Finally we give the proof Theorem B.2. 
Proof of Theorem B.2 Since $G$ is second countable, it is a Lindelöf space, ie, every open covering contains a countable subcovering; see [27, Theorem 1.3, Section 4-1, page 191].

By Theorem B.6 we know that the orbit $G \cdot \alpha$ is closed in $\operatorname{hom}(H, G)$. We have already explained that $\operatorname{hom}(H, G)$ is a complete metric space. We conclude that the orbit $G \cdot \alpha$ is a complete metric space and hence by the Baire Category Theorem (see [27, Theorem 7.2, Section 7.7, page 294]) also a Baire space, ie every countable union of closed sets each of which has empty interior has itself empty interior.

Next we show that the map $\iota_{\alpha}$ is an open map. Since we know that it is a bijective continuous $G$-map, this will imply that $\iota_{\alpha}$ is indeed a $G$-homeomorphism. Since the image and source of $\iota_{\alpha}$ are transitive $G$-spaces, it suffices to show for every open set $U$ of $G$ containing $1_{G} \in G$ that there exists an open neighborhood of $\alpha$ which is contained in $\iota_{\alpha}(U)$.

Since $G$ is locally compact and the map $\mu: G \times G \rightarrow G,\left(g_{1}, g_{2}\right) \mapsto g_{1}^{-1} g_{2}$ is continuous, we can find a nonempty open set $V \subset U$ such that its closure $K=\bar{V}$ is compact and satisfies

$$
K^{-1} K:=\left\{k_{1}^{-1} \cdot k_{2} \mid k_{1}, k_{2} \in K\right\} \subset U ;
$$

any nonempty open set $V \subset U$ such that $\bar{V} \times \bar{V} \subset \mu^{-1}(U) \cap U \times U$ satisfies this condition.

Since $G$ is a Lindelöf space, it can be covered by countably many $G$-translates of the open subset $V$ and hence also by countably many $G$-translates of the subset $K$. This implies that $G / C_{G}(\alpha)$ can be covered by countably many $G$-translates of $K C_{G}(\alpha) / C_{G}(\alpha)$. Since each

$$
\iota_{\alpha}\left(g K C_{G}(\alpha) / C_{G}(\alpha)\right)=g K \cdot \alpha
$$

is a closed set in $G \cdot \alpha$ and $G \cdot \alpha$ is a Baire space, one of the $G$-translates of $K C_{G}(\alpha) / C_{G}(\alpha)$ must have nonempty interior. This implies that $K \cdot \alpha$ has nonempty interior, and therefore the set $K^{-1} K \cdot \alpha$ contains an open neighborhood of $\alpha$. Since $K^{-1} K \alpha$ is contained in $i_{\alpha}(U)$, the claim follows.

\section{References}

[1] H Abels, A universal proper G-space, Math. Z. 159 (1978) 143-158 MR0501039

[2] MF Atiyah, K-theory and reality, Quart. J. Math. Oxford Ser. 17 (1966) 367-386 MR0206940 
[3] M F Atiyah, Algebraic topology and operators in Hilbert space, from: "Lectures in Modern Analysis and Applications, I", (C T Taam, editor), Springer, Berlin (1969) 101-121 MR0248803

[4] M F Atiyah, GB Segal, Twisted K-theory, Ukr. Mat. Visn. 1 (2004) 287-330 MR2172633

[5] T Barcenas, J Espinoza, M Joachim, B Uribe, Classification of twists in equivariant $K$-theory for proper and discrete group actions, Proc. Lond. Math. Soc. (2013)

[6] G Birkhoff, A note on topological groups, Compositio Math. 3 (1936) 427-430 MR1556955

[7] T tom Dieck, Transformation groups, Studies in Mathematics 8, de Gruyter, Berlin (1987) MR889050

[8] S A Gaal, Linear analysis and representation theory, Grundlehren der Math. Wissenschaften 198, Springer, New York (1973) MR0447465

[9] I Hambleton, J-C Hausmann, Equivariant principal bundles over spheres and cohomogeneity one manifolds, Proc. London Math. Soc. 86 (2003) 250-272 MR1971468

[10] P de la Harpe, Classical Banach-Lie algebras and Banach-Lie groups of operators in Hilbert space, Lecture Notes in Mathematics 285, Springer, Berlin (1972)

[11] D Husemoller, Fibre bundles, McGraw-Hill Book Co., New York (1966) MR0229247

[12] S Illman, Existence and uniqueness of equivariant triangulations of smooth proper $G$-manifolds with some applications to equivariant Whitehead torsion, J. Reine Angew. Math. 524 (2000) 129-183 MR1770606

[13] K Jänich, Vektorraumbündel und der Raum der Fredholm-Operatoren, Math. Ann. 161 (1965) 129-142 MR0190946

[14] S Kakutani, Über die Metrisation der topologischen Gruppen, Proc. Imp. Acad. 12 (1936) 82-84 MR1568424

[15] N Kitchloo, Dominant $K$-theory and integrable highest weight representations of Kac-Moody groups, Adv. Math. 221 (2009) 1191-1226 MR2518636

[16] N H Kuiper, The homotopy type of the unitary group of Hilbert space, Topology 3 (1965) 19-30 MR0179792

[17] S Lang, Differential manifolds, Addison-Wesley, Reading, MA (1972) MR0431240

[18] R K Lashof, Equivariant bundles, Illinois J. Math. 26 (1982) 257-271 MR650393

[19] R K Lashof, J P May, Generalized equivariant bundles, Bull. Soc. Math. Belg. Sér. A 38 (1986) 265-271 MR885537

[20] R K Lashof, J P May, GB Segal, Equivariant bundles with abelian structural group, from: "Proceedings of the Northwestern Homotopy Theory Conference", (H R Miller, S B Priddy, editors), Contemp. Math. 19, Amer. Math. Soc. (1983) 167-176 MR711050 
[21] D H Lee, T S Wu, On conjugacy of homomorphisms of topological groups, II, Illinois J. Math. 14 (1970) 409-413 MR0267037

[22] W Lück, Transformation groups and algebraic $K$-theory, Lecture Notes in Mathematics 1408, Springer, Berlin (1989) MR1027600

[23] W Lück, Survey on classifying spaces for families of subgroups, from: "Infinite groups: geometric, combinatorial and dynamical aspects", (L Bartholdi, T CeccheriniSilberstein, T Smirnova-Nagnibeda, A Zuk, editors), Progr. Math. 248, Birkhäuser (2005) 269-322 MR2195456

[24] J P May, Some remarks on equivariant bundles and classifying spaces, from: "International Conference on Homotopy Theory”, Astérisque 191, Soc. Math. France, Paris (1990) 7, 239-253 MR1098973

[25] H Miyazaki, The paracompactness of CW-complexes, Tôhoku Math. J. 4 (1952) 309-313 MR0054246

[26] PS Mostert, Local cross sections in locally compact groups, Proc. Amer. Math. Soc. 4 (1953) 645-649 MR0056614

[27] J R Munkres, Topology: A first course, Prentice-Hall, Englewood Cliffs, N.J. (1975) MR0464128

[28] M Murayama, K Shimakawa, Universal equivariant bundles, Proc. Amer. Math. Soc. 123 (1995) 1289-1295 MR1231040

[29] K-H Neeb, Towards a Lie theory of locally convex groups, Jpn. J. Math. 1 (2006) 291-468 MR2261066

[30] R S Palais, On the existence of slices for actions of noncompact Lie groups, Ann. of Math. 73 (1961) 295-323 MR0126506

[31] G B Segal, Cohomology of topological groups, from: "Symposia Mathematica, Vol. IV”, Academic Press, London (1970) 377-387 MR0280572

[32] D J Simms, Topological aspects of the projective unitary group, Proc. Cambridge Philos. Soc. 68 (1970) 57-60 MR0261394

[33] N E Steenrod, A convenient category of topological spaces, Michigan Math. J. 14 (1967) 133-152 MR0210075

Mathematisches Institut, Rheinische Wilhelms-Universität Bonn

Endenicher Allee 62, 53115 Bonn, Germany

Departamento de Matemáticas, Universidad del Norte

Km.5 Vía Puerto Colombia, Barranquilla, Colombia

wolfgang.lueck@him.uni-bonn.de, buribe@gmail.com

http://www.him.uni-bonn.de/lueck/

Received: 17 April 2013 Revised: 7 January 2014 
